\title{
CAMA
}

Centre for Applied Macroeconomic Analysis

\section{Inflation Dynamics: The Role of Public Debt and Policy Regimes}

\section{CAMA Working Paper 75/2013 November 2013}

\section{Saroj Bhattarai}

Department of Economics, Pennsylvania State University

\section{Jae Won Lee}

Department of Economics, Seoul National University

\section{Woong Yong Park}

Faculty of Business and Economics, University of Hong Kong and Centre for Applied Macroeconomics Analysis

\section{Abstract}

We investigate the roles of a time-varying inflation target and monetary and fiscal policy stances on the dynamics of inflation in a DSGE model. Under an active monetary and passive fiscal policy regime, inflation closely follows the path of the inflation target and a stronger reaction of monetary policy to inflation decreases the response of inflation to non-policy shocks. In sharp contrast, under an active fiscal and passive monetary policy regime, inflation moves in an opposite direction from the inflation target and a stronger reaction of monetary policy to inflation increases the response of inflation to non-policy shocks. Moreover, a higher level of government debt leads to a greater response of inflation while a weaker response of fiscal policy to debt decreases the response of inflation to non-policy shocks. These results are due to variation in the value of public debt that leads to wealth effects on households. Finally, under a passive monetary and passive fiscal policy regime, both monetary and fiscal policy parameters matter for inflation dynamics, but because of equilibrium indeterminacy, theory provides no clear answer on the overall behavior of inflation. We characterize these results analytically in a simple model and numerically in a quantitative model. 


\section{Keywords}

Time-varying inflation target, Inflation response, Public debt, Monetary and fiscal policy regimes, Monetary and fiscal policy stances, DSGE model

\section{JEL Classification}

E31, E52, E63

\section{Address for correspondence:}

(E) cama.admin@anu.edu.au

The Centre for Applied Macroeconomic Analysis in the Crawford School of Public Policy has been established to build strong links between professional macroeconomists. It provides a forum for quality macroeconomic research and discussion of policy issues between academia, government and the private sector.

The Crawford School of Public Policy is the Australian National University's public policy school, serving and influencing Australia, Asia and the Pacific through advanced policy research, graduate and executive education, and policy impact. 


\title{
Inflation Dynamics: The Role of Public Debt and Policy Regimes*
}

\author{
Saroj Bhattarai, Jae Won Lee; and Woong Yong Park ${ }^{\S}$
}

\begin{abstract}
We investigate the roles of a time-varying inflation target and monetary and fiscal policy stances on the dynamics of inflation in a DSGE model. Under an active monetary and passive fiscal policy regime, inflation closely follows the path of the inflation target and a stronger reaction of monetary policy to inflation decreases the response of inflation to non-policy shocks. In sharp contrast, under an active fiscal and passive monetary policy regime, inflation moves in an opposite direction from the inflation target and a stronger reaction of monetary policy to inflation increases the response of inflation to non-policy shocks. Moreover, a higher level of government debt leads to a greater response of inflation while a weaker response of fiscal policy to debt decreases the response of inflation to non-policy shocks. These results are due to variation in the value of public debt that leads to wealth effects on households. Finally, under a passive monetary and passive fiscal policy regime, both monetary and fiscal policy parameters matter for inflation dynamics, but because of equilibrium indeterminacy, theory provides no clear answer on the overall behavior of inflation. We characterize these results analytically in a simple model and numerically in a quantitative model.
\end{abstract}

JEL Classification: E31, E52, E63

Keywords: Time-varying inflation target, Inflation response, Public debt, Monetary and fiscal policy regimes, Monetary and fiscal policy stances, DSGE model

*We are grateful to Seonghoon Cho, Ricardo Reis, Felipe Schwartzman, Neil Wallace, Tack Yun, seminar participants at University of Hong Kong and Bank of Korea, and conference participants at the $15^{\text {th }}$ KEA International Conference and the 2013 Society of Economic Dynamics Annual Meeting for comments and criticisms. We thank Seven Liu for research assistance. This version: November 2013.

${ }^{\dagger}$ Department of Economics, Pennsylvania State University. Email: sub31@psu.edu.

$\ddagger$ Department of Economics, Seoul National University. Email: jwlee7@snu.ac.kr.

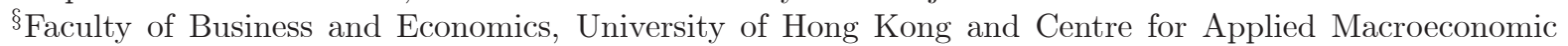
Analysis (CAMA), Australian National University. Email: wypark@hku.hk. 


\section{Introduction}

Using a micro-founded model, we address three classic questions in monetary economics and policy in this paper. First, does monetary policy always properly control the dynamics and path of inflation? Specifically, can a time-varying inflation target decisively influence the path of actual inflation? Second, does the level of public debt affect inflation dynamics? In particular, does a higher level of public debt lead to a greater equilibrium response of inflation? Third, what are the roles of monetary and fiscal policy stances on inflation dynamics and the effects of changes in policy stances on the equilibrium response of inflation to various shocks impinging on the economy? For example, what happens to the equilibrium behavior of inflation when the monetary policy stance changes to a more aggressive response to inflation? Does the fiscal policy stance with respect to public debt matter for inflation dynamics? If yes, then how does a variation in the fiscal policy stance affect inflation?

These issues, while long of great interest in monetary economics, have received a renewed interest recently. A prominent illustration is the research that aims to provide an explanation to the rise of U.S. inflation in the $1970 \mathrm{~s}$ and its subsequent fall in the $1980 \mathrm{~s}$. Proposed explanations typically rely on changes in the dynamics of the inflation target and/or changes in policy stances: Ireland (2007) and Cogley, Primiceri, and Sargent (2010) propose a rise in a persistent time-varying inflation target as an explanation for the rise of inflation in the 1970s; Clarida, Gali, and Gertler (2000), Lubik and Schorfheide (2004), and Bhattarai, Lee, and Park (2012a and 2012b) argue that a weak monetary policy stance with respect to inflation in the pre-Volcker period implied indeterminacy of equilibria, which in turn, increased inflation due to self-fulfilling beliefs; while Sims (2011) and Bianchi and Ilut (2012) argue that a weak response of taxes to debt led to an increase in inflation in the $1970 \mathrm{~s}$ as a response to increases in government spending. ${ }^{1}$ Moreover, rising levels of public debt in many countries have recently raised concerns on such a development leading to future inflation.

Motivated by these considerations, in the first part of the paper, we provide a complete and analytical characterization of these questions in a simple sticky-price dynamic stochastic general equilibrium (DSGE) model with feedback policy rules. We analyze three different policy regimes. The first policy regime is an active monetary and passive fiscal policy regime, where a high response of interest rates to inflation is coupled with a high response of taxes to outstanding public debt. ${ }^{2}$ This is the most common policy regime considered in the literature where a unique stable equilibrium exists. In this regime, inflation closely follows the path of the inflation target and stronger the systematic reaction of monetary policy to inflation, more

\footnotetext{
${ }^{1}$ Some well-known papers, such as Primiceri (2006) and Sargent, Williams, and Zha (2006), provide a learning-based explanation for the rise and fall of U.S. inflation. Others, such as Sims and Zha (2006), have attributed the rise and fall of U.S. inflation to time-varying volatility of shocks.

${ }^{2}$ We use the language of Leeper (1991) in characterizing policies as active and passive.
} 
closely will actual inflation follow the inflation target. Finally, the level of public debt and the fiscal policy stance plays no role in price level determination. These results are standard since in this regime, monetary policy controls inflation dynamics.

Second, we analyze an active fiscal and passive monetary policy regime, where a low response of interest rates to inflation is coupled with a low response of taxes to outstanding public debt. ${ }^{3}$ A unique stable equilibrium exists with this combination of monetary and fiscal policies as well. In this regime, in sharp contrast to the previous regime, inflation moves in an opposite direction from the inflation target on impact. In fact, stronger the systematic reaction of monetary policy to inflation, greater will be the divergence between the inflation target and actual inflation. In addition, and again in sharp contrast to the active monetary and passive fiscal regime, a stronger reaction of monetary policy to inflation increases the response of inflation to various non-policy shocks and raises inflation volatility. Moreover, now, fiscal policy - the level of public debt and the fiscal policy stance - matters for inflation dynamics. In particular, a higher level of public debt leads to an increase in inflation, the magnitude of which rises as fiscal policy becomes more active (i.e. a weaker response of taxes to debt). In addition, we show that a more active fiscal policy leads to a weaker response of inflation to non-policy shocks.

These results arise because of the wealth effect on households as the value of outstanding government debt varies due to interest rate and tax changes. ${ }^{4}$ Under the active monetary and passive fiscal policy regime, because the systematic response of interest rates to inflation was greater than one, expected inflation decreases in response to an unanticipated increase in interest rates. In this regime, in contrast, when monetary policy raises interest rates whether responding to a decrease in the inflation target or to other non-policy shocks - the value of government debt rises to cover the increased interest expense. This leads to a positive wealth effect on those who hold government debt, the households, because they perceive this increase in value of government debt as increasing their wealth since it is not matched by tax increases that are enough to satisfy the intertemporal government budget constraint at prevailing prices. The positive wealth effect then leads to increased spending by households which in turn increases inflation in equilibrium. Thus, inflation will move in the opposite direction from the lowered inflation target. Moreover, greater the systematic response of interest rates to inflation, as long as this response is less than one-for-one, it only serves to make this positive wealth effect stronger. Then, the equilibrium response of inflation to both

\footnotetext{
${ }^{3}$ For early treatments of this policy regime in simple models, see among others, Leeper (1991), Sims (1994), and Woodford (1995). Kim (2003) and Canzoneri, Cumby, and Diba (2011) present numerical results on the effects of some shocks in a sticky-price model under this policy regime. Our contribution is an analytical characterization of both the solution as well as the comparative statics with respect to policy parameters.

${ }^{4}$ As we explain in detail in the paper, this mechanism is distinct from the classic mechanism proposed by Sargent and Wallace (1981) that is related to monetization of public debt.
} 
policy and non-policy shocks will be even higher.

Similarly, fiscal policy matters for inflation dynamics as first, a higher level of public debt leads to an increase in inflation as it leads to a higher wealth effect on households. Second, fiscal policy stance also affects inflation, but unlike monetary policy stance, it affects household wealth by controlling directly the magnitude of the change in tax revenues - rather than interest expenses - after a shock. In particular, when non-policy shocks hit the economy, the resulting changes in lump-sum taxes cause a wealth effect on households, again because tax changes do not respond strongly enough to debt. ${ }^{5}$ Moreover, weaker the response of taxes to debt, lower is this wealth effect. Thus, spending and thereby inflation, responds by less to both the level of debt outstanding as well as non-policy shocks.

Third, we explore a passive monetary and passive fiscal policy regime, where a low response of interest rates to inflation is coupled with a high response of taxes to outstanding government debt. In this regime, there is equilibrium indeterminacy and both fundamental and sunspot shocks affect inflation. Importantly, generally, both monetary and fiscal policy stances matter for inflation dynamics, although the level of government debt does not affect inflation. Due to the potential for self-fulfilling beliefs however, theory provides no clear guidance on the overall behavior of inflation.

While at first we provide closed-form solutions for a simple model, in the second part of the paper, we also conduct a quantitative experiment with a richer DSGE model that includes a variety of shocks and frictions. We show that for realistic parameter values, our analytical results continue to apply in such a model.

Our results have implications for both the empirical and theoretical literature in monetary economics. ${ }^{6}$ For example, consider the recent practice in papers that estimate monetary DSGE models of using a time-varying inflation target process to explain the low frequency movement in actual inflation. In particular, Ireland (2007) and Cogley, Primiceri, and Sargent (2010) show that the estimated inflation target tracks actual inflation remarkably well in post-WWII U.S. data. Moreover, in a recent comprehensive study of various monetary policy reaction functions, Curdia, Ferrero, Ng, and Tambalotti (2011) show that using a persistent timevarying inflation target improves the fit of the model since it helps capture the low frequency variation in inflation. Our results show that this strategy works only if one imposes an active monetary and passive fiscal policy regime while estimating the model as well as ensures that the inflation target shock is more persistent than other non-policy shocks. ${ }^{7}$ Indeed, using an estimated DSGE model and a pre-Volcker and a post-Volcker subsample analysis, Bhattarai,

\footnotetext{
${ }^{5}$ Changes in lump-sum taxes thus affect consumption in this economy due to the wealth effect.

${ }^{6}$ For a recent survey of the literature on monetary and fiscal policy interactions, see Canzoneri, Cumby, and Diba (2011).

${ }^{7}$ Curdia, Ferrero, Ng, and Tambalotti (2011) use U.S. data from 1987 : III to 2009 : III, a period during which an active monetary and passive fiscal policy regime is certainly a reasonable description of policy.
} 
Lee, and Park (2012b) show that the correlation between inflation and the smoothed inflation target backed out after estimation varies significantly depending on which policy regime one imposes during estimation. Figure 1, reproduced from that paper, makes the point clear as it shows that while under an active monetary and passive fiscal policy regime, the long-run correlation between the inflation target and actual inflation is high and positive, under a passive monetary and active fiscal policy regime, it is strongly negative. Moreover, under a passive monetary and passive fiscal policy regime, while theoretically the correlation between the inflation target and actual inflation is not pinned down, empirically, the correlation is close to zero.

Our theoretical results show that under a passive monetary and passive fiscal policy regime, while public debt is not a state variable, both monetary and fiscal policy stances matter for inflation dynamics. Thus, in bringing a model under indeterminacy to the data, dropping fiscal policy from the model is a source of misspecification. We thus derive new results by generalizing Lubik and Schorfheide (2003), who did not specify fiscal policy explicitly. Finally, we show that the effects of an aggressive monetary policy stance, or a "hawkish" central bank, on inflation depends critically on the joint behavior of monetary and fiscal policy. In particular, we establish that in a passive monetary and active fiscal policy regime, an aggressive reaction to inflation by the central bank actually ends up destabilizing inflation in response to nonpolicy shocks. Thus, any prescription for monetary policy behavior has to take into account the prevailing fiscal policy regime. ${ }^{8}$

\section{Simple Model}

For our analytical analysis, we consider a prototypical DSGE model with nominal rigidities. ${ }^{9}$ The log-linearized model around zero-inflation steady state can be summarized by:

$$
\begin{aligned}
\tilde{Y}_{t} & =E_{t} \tilde{Y}_{t+1}-\left(\hat{R}_{t}-E_{t} \hat{\pi}_{t+1}\right)+\hat{r}_{t}^{*} \\
\hat{\pi}_{t} & =\kappa \tilde{Y}_{t}+\beta E_{t} \hat{\pi}_{t+1} \\
\hat{R}_{t} & =\phi\left(\hat{\pi}_{t}-\hat{\pi}_{t}^{*}\right) \\
\hat{\tau}_{t} & =\psi \hat{b}_{t-1} \\
\hat{b}_{t} & =\beta^{-1} \hat{b}_{t-1}-\beta^{-1} \bar{b} \hat{\pi}_{t}-\beta^{-1} \hat{\tau}_{t}+\bar{b} \hat{R}_{t}
\end{aligned}
$$

\footnotetext{
${ }^{8}$ Loyo (1999) uses a similar result from a flexible price model to interpret the experience of Brazil in the 1970s and 1980s and shows that a strong response of interest rates to inflation can lead to a hyperinflationary spiral under a passive monetary and active fiscal policy regime. Here, we work with a determinate equilibrium in a sticky-price model. Relatedly, Sims (2004) shows in a very different set-up, also a flexible price model, that a central bank might lose control of inflation if it is not adequately backed up by the treasury.

${ }^{9}$ Since this model is well-known, we provide a complete model description in the appendix.
} 


$$
\begin{aligned}
\hat{r}_{t}^{*} & =\rho_{r} \hat{r}_{t-1}^{*}+\varepsilon_{r, t}, \\
\hat{\pi}_{t}^{*} & =\rho_{\pi} \hat{\pi}_{t-1}^{*}+\varepsilon_{\pi, t},
\end{aligned}
$$

where $\tilde{Y}_{t}$ is the output gap, the difference between actual output $\hat{Y}_{t}$ and the output that would prevail under flexible prices, $\hat{R}_{t}$ is the nominal interest rate, $\hat{\pi}_{t}$ is inflation, and $\hat{r}_{t}^{*}$ - often referred to as the natural rate of interest - is a composite shock that is a linear combination of the structural shocks. In particular, we show in the appendix that demand-type shocks such as preference shocks increase $\hat{r}_{t}^{*}$, while supply-type shocks such as technology shocks lower $\hat{r}_{t}^{*}$. Equations (1) and (2) represent the intertemporal Euler equation and Phillips curve, respectively, that are standard in monetary DSGE models. Here, $\beta$ is the discount factor of the household and $\kappa$, the slope of the Phillips curve, is a composite parameter of the structural parameters. $^{10}$

Monetary policy is modelled using an interest rate rule (3) that features a systematic response of the nominal interest rate $\hat{R}_{t}$, with feedback parameter $\phi$, to the deviation of inflation $\hat{\pi}_{t}$ from a time-varying target $\hat{\pi}_{t}^{*}$. Fiscal policy is modelled using a tax rule (4) that features a systematic response of the tax revenues $\hat{\tau}_{t}$, with feedback parameter $\psi$, to the real maturity value of outstanding government debt $\hat{b}_{t-1}$. For simplicity, we assume that the government issues one-period nominal debt and levies lump-sum taxes. Finally, Equation (5) is the flow budget constraint of the government.

We assume in Equations (6) and (7) that both $\hat{r}_{t}^{*}$ and $\hat{\pi}_{t}^{*}$ follow a stationary $\operatorname{AR}(1)$ process with i.i.d. innovations. Ireland (2007) models that the Federal Reserve adjusts the inflation target in response to the economy's supply shocks but finds that the response is not statistically significant. In light of this result, we make the exogeneity assumption on $\hat{\pi}_{t}^{*}$. Cogley and Sbordone (2008) and Cogley, Primiceri, and Sargent (2010) also use an exogenous AR process to model the inflation target. ${ }^{11}$

As is well-known, the existence and uniqueness of equilibrium depends crucially on the prevailing monetary and fiscal policy regime. The equilibrium of the economy will be determinate either if monetary policy is active while fiscal policy is passive or if monetary policy is

\footnotetext{
${ }^{10}$ We use $\hat{X}_{t}$ to denote the $\log$ deviation of a variable $X_{t}$ from its steady state $\bar{X}$, except for two fiscal variables, $\hat{b}_{t}$ and $\hat{\tau}_{t}$. Following Woodford (2003), we let them represent respectively the deviation of the maturity value of government debt and of government tax revenues (net of transfers) from their steady-state levels, measured as a fraction of steady-state output: $\hat{b}_{t}=\left(b_{t}-\bar{b}\right) / \bar{Y}$ and $\hat{\tau}_{t}=\left(\tau_{t}-\bar{\tau}\right) / \bar{Y}$.

${ }^{11}$ The assumption that $\hat{\pi}_{t}^{*}$ is stationary implies that the monetary authority does not permanently keep the inflation target at a constant level but in the long run drives the inflation target back to the steady state level, zero. We introduce this assumption for two reasons. First, the stationarity assumption allows us to work with a standard framework. To assume that the inflation target has a unit root results in time-varying coefficients of the Phillips curve as in Cogley and Sbordone (2008) or leads to a non-standard monetary policy rule for with which the Taylor principle should be modified. Second, we will generally restrict $\rho_{\pi}$ to values close to 1 , thereby effectively ensuring that $\hat{\pi}_{t}^{*}$ captures the persistent behavior of the inflation target set by the central bank. Cogley, Primiceri, and Sargent (2010) use the same assumption.
} 
passive while fiscal policy is active. The equilibrium is indeterminate and multiple equilibria exist if both monetary and fiscal policies are passive while no stable equilibrium exists if both monetary and fiscal policies are active. In our model, monetary policy is active if $\phi>1$ and fiscal policy is active if $\psi<1-\beta$.

\section{$2.1 \quad$ Results}

We analytically characterize the solution of the model either when a determinate equilibrium exists or when there are multiple equilibria. We then derive several results regarding the dynamics of inflation. Specifically, we study how the path of inflation depends on the path of the inflation target, whether the level of debt outstanding matters for the behavior of inflation, and how the response of inflation changes when monetary and fiscal policy stances change within a policy regime. All the details of the derivations and the proofs of the various propositions are provided in the appendix.

\subsubsection{Active Monetary and Passive Fiscal Policy}

Under an active monetary and passive fiscal (AMPF) policy regime, the solution for inflation is:

$$
\hat{\pi}_{t}=\Phi(\phi) \hat{\pi}_{t}^{*}+\Gamma(\phi) \hat{r}_{t}^{*}
$$

where $\Phi(\phi)$ and $\Gamma(\phi)$ are functions of the monetary policy response parameter $\phi .{ }^{12}$ Note that debt $\hat{b}_{t-1}$ is not a state variable and thus inflation is only a function of the exogenous shocks $\hat{\pi}_{t}^{*}$ and $\hat{r}_{t}^{*}$. Moreover, the fiscal policy response parameter $\psi$ does not affect inflation.

We next characterize several properties of the solution. We first start with the response of inflation to changes in the inflation target and the non-policy shock.

Proposition 1 (Direction of inflation response) Under AMPF, inflation moves in the same direction as the inflation target $\hat{\pi}_{t}^{*}$. Moreover, inflation responds more (or less) than one-for-one to changes in the inflation target if prices are sufficiently flexible (or sticky):

$$
0<\Phi(\phi) \leq 1 \quad \forall \kappa \in(0, \bar{\kappa}], \quad \text { and } \quad \Phi(\phi)>1 \quad \forall \kappa \in(\bar{\kappa}, \infty)
$$

where $\bar{\kappa} \equiv\left(1-\rho_{\pi}\right)\left(1-\beta \rho_{\pi}\right) / \rho_{\pi}$. Finally, inflation moves in the same direction in response to the non-policy shock, $\hat{r}_{t}^{*}$ - that is,

$$
\Gamma(\phi)>0 .
$$

\footnotetext{
${ }^{12}$ Obviously, $\Phi(\phi)$ and $\Gamma(\phi)$ in Equation (8) are a function of other structural parameters as well. From here on after, we write the coefficients in a solution for inflation as a function of policy parameters only so as to highlight their role in determining inflation dynamics.
} 
In this regime, since inflation moves in the same direction as the inflation target, we see clearly that monetary policy controls the dynamics of inflation. As is natural, the response of inflation to the inflation target shock depends on the extent of price stickiness in the economy: smaller the degree of price stickiness, greater is the response of inflation. In particular, when prices are sufficiently flexible $(\kappa>\bar{\kappa})$, inflation overshoots the central bank's target rate. ${ }^{13}$ Proposition 1 also establishes that inflation moves in the same direction as the natural rate of interest $\hat{r}_{t}^{*}$. Hence, inflation increases in response to favorable demand shocks and decreases in response to favorable supply shocks. This is a conventional result under the AMPF regime.

Next, we consider a comparative static exercise with respect to the policy parameter $\phi$, which is the measure of the monetary policy stance.

Proposition 2 (Magnitude of inflation response and monetary policy stance) Under $A M P F$, the response of inflation to changes in the inflation target is decreasing (or increasing) in $\phi$ if prices are sufficiently flexible (or sticky):

$$
\frac{\partial \Phi(\phi)}{\partial \phi} \geq 0 \quad \forall \kappa \in(0, \bar{\kappa}], \quad \text { and } \quad \frac{\partial \Phi(\phi)}{\partial \phi}<0 \quad \forall \kappa \in(\bar{\kappa}, \infty)
$$

The equality holds when $\kappa=\bar{\kappa}$. Moreover, inflation responds less to non-policy shocks as the monetary authority becomes more aggressive - that is, $\Gamma$ decreases as $\phi$ increases:

$$
\frac{\partial \Gamma(\phi)}{\partial \phi}<0
$$

In combination with Proposition 1, we now have the intuitive result that $\hat{\pi}_{t}$ will deviate less from $\hat{\pi}_{t}^{*}$ as $\phi$ increases - for all values of $\kappa$ and after all types of shocks (policy and non-policy). Moreover, due to the absence of an endogenous state variable in Equation (8), the long-run variance of the "inflation gap," $\hat{\pi}_{t}-\hat{\pi}_{t}^{*}$, decreases when the monetary authority reacts systematically strongly to inflation: $\partial V A R\left(\hat{\pi}_{t}-\hat{\pi}_{t}^{*}\right) / \partial \phi<0$. Thus, if the central bank's objective is to stabilize the inflation gap, a more aggressive monetary policy stance is desired.

We conclude this subsection with a note that our results reveal an interesting point and provide another interpretation of some recent results in the literature. Empirical studies such as Cogley, Primiceri, and Sargent (2010), which specify active monetary policy, have found that the low-frequency components of the inflation rate are explained almost entirely by a time-varying inflation target. The literature's strategy is to calibrate $\rho_{\pi}$ to a large value, close to a random walk. Our results - that $\hat{\pi}_{t}$ moves closely with $\hat{\pi}_{t}^{*}-$ are consistent with those

\footnotetext{
${ }^{13}$ While inflation in theory can either overshoot or undershoot variations in the inflation target, the model in practice almost always generates "over-shooting" because the lower bound of $\kappa$ for $\Phi(\phi)>1$, found in Proposition 1 , is very small when $\rho_{\pi}$ has a value close to one, which is usually the case in the literature. For example, at our benchmark parameterization $\left(\beta=0.99 ; \rho_{\pi}=0.995\right), \bar{\kappa}$ is less than 0.0001 .
} 
findings. Moreover, as is clear from Equation (8), to the extent that the inflation target is more persistent than other exogenous variables $\left(\rho_{\pi}>\rho_{r}\right)$, the inflation target - relative to other shocks - will dominate inflation dynamics, especially at low frequencies.

We now analyze another policy regime that leads to a determinate equilibrium.

\subsubsection{Passive Monetary and Active Fiscal Policy}

Under a passive monetary and active fiscal (PMAF) policy regime, the solution for inflation is:

$$
\hat{\pi}_{t}=\Omega(\phi, \psi) \hat{b}_{t-1}-\Phi(\phi, \psi) \hat{\pi}_{t}^{*}+\Gamma(\phi, \psi) \hat{r}_{t}^{*},
$$

where $\Omega(\phi, \psi), \Phi(\phi, \psi)$, and $\Gamma(\phi, \psi)$ are functions of both the monetary policy response parameter $\phi$ and the fiscal policy response parameter $\psi$. Note that the dynamics of inflation depend on public debt outstanding $\hat{b}_{t-1}$, which is an endogenous state variable in the model. This is an important difference from the case we analyzed under AMPF. As we explain below, the wealth effect on households due to changes in the value of government debt and taxes, which in turn affects households' spending, is the main mechanism behind our results in this section.

We next characterize several properties of the solution. We first start with the response of inflation to changes in the inflation target, the non-policy shock, and public debt outstanding.

Proposition 3 (Direction of inflation response) Under PMAF, inflation moves in the opposite direction in response to a change in the inflation target - that is,

$$
\Phi(\phi, \psi) \geq 0 \quad \text { for } \psi \in\left(-\infty, \bar{\psi}^{*}\right) \text { and } \phi \in[0,1)
$$

where $0<\bar{\psi}^{*} \leq 1-\beta$ is a reduced-form parameter. The equality holds when $\phi=0$. However, inflation moves in the same direction in response to the non-policy shock, $\hat{r}_{t}^{*}$ - that is,

$$
\Gamma(\phi, \psi)>0 \quad \text { for } \psi \in(-\infty, 1-\beta) \text { and } \phi \in[0,1)
$$

Finally, inflation moves in the same direction in response to a change in public debt outstanding - that is,

$$
\Omega(\phi, \psi)>0 \quad \text { for } \psi \in(-\infty, 1-\beta) \text { and } \phi \in[0,1)
$$

The result of Proposition 3 on the negative relationship between inflation and the inflation target, which is in stark contrast to Proposition 1 under the AMPF regime, arises because now changes in the value of government debt influence inflation dynamics. ${ }^{14}$ Suppose that

\footnotetext{
${ }^{14}$ Note that for the first result of this proposition, there is a condition on $\psi$ such that fiscal policy has to
} 
the monetary authority unexpectedly lowers the inflation target. From Equation (3), this increases the nominal interest rate on impact. An increase in the nominal interest rate results in an increase of the value of government debt, due to an increase in interest expense. In this active fiscal policy regime, since taxes do not adjust by enough to satisfy the intertemporal government budget constraint at prevailing prices, the increase in the value of government debt leads to a positive wealth effect on households who hold government debt. This positive wealth effect then leads to higher spending, which pushes up inflation in equilibrium. Proposition 3 is therefore, the key reason behind the negative relationship between inflation and the inflation target under the PMAF policy regime shown in Figure 1.

Proposition 3 also establishes that under a PMAF regime, inflation moves in the same direction as the non-policy shock. This result is the same as Proposition 1 under the AMPF regime because even under the PMAF regime, the effect of a positive non-policy shock on the economy is still to increase the output gap given the expectations as implied by Equation (1), and in turn, inflation as implied by Equation (2).

Finally, Proposition 3 also shows that under a PMAF policy regime, inflation is affected positively by changes in public debt outstanding $\hat{b}_{t-1}$. This is also in stark contrast to the AMPF regime. This result is again a direct derivative of the wealth effect on households discussed above that is a crucial mechanism under active fiscal policy. A higher level of public debt outstanding, not matched by sufficient tax increases, is translated into higher wealth for households, which increases spending and thereby inflation. Thus, even with lump-sum taxes, a higher level of outstanding government debt is inflationary in this regime.

Next we consider a comparative static exercise with respect to the policy parameter $\phi$.

Proposition 4 (Magnitude of inflation response and monetary policy stance) Under $P M A F$, inflation deviates even further from the inflation target when the monetary authority is more aggressive - that is, $\Phi$ increases as $\phi$ increases in the domain of $[0,1)$ :

$$
\frac{\partial \Phi(\phi, \psi)}{\partial \phi}>0 \quad \text { for } \psi \in\left(-\infty, \bar{\psi}^{* *}\right) \text { and } \phi \in[0,1)
$$

where $0<\bar{\psi}^{* *} \leq 1-\beta$ is a reduced-form parameter. Moreover, inflation responds more to non-policy shocks when the monetary authority is more aggressive-that is, $\Gamma$ increases as $\phi$ increases in the domain of $[0,1)$ :

$$
\frac{\partial \Gamma(\phi, \psi)}{\partial \phi}>0 \quad \text { for } \psi \in(-\infty, 1-\beta) \text { and } \phi \in[0,1)
$$

be "sufficiently" active. That is, $\psi<\bar{\psi}^{*} \leq 1-\beta$. As we argue in the appendix, this condition is unlikely to be relevant empirically. 
Finally, inflation responds more to a change in public debt outstanding when the monetary authority is more aggressive - that is, $\Omega$ increases as $\phi$ increases in the domain of $[0,1)$ :

$$
\frac{\partial \Omega(\phi, \psi)}{\partial \phi}>0 \quad \text { for } \psi \in\left(-\infty, \bar{\psi}^{* * *}\right) \text { and } \phi \in[0,1)
$$

where $0<\bar{\psi}^{* * *} \leq 1-\beta$ is a reduced-form parameter.

In sharp contrast to our result under the AMPF regime in Proposition 2, Proposition 4 shows that under a PMAF regime, as the reaction of monetary policy to inflation increases, so does the impact on inflation of a change in the inflation target. ${ }^{15}$ This is because when the reaction of monetary policy to inflation increases, for a given decrease in the inflation target, the nominal interest rate increase will be higher. This means that the value of government debt increases by more, which in turn, increases the size of the wealth effect discussed above. This then implies a greater effect on spending, and thereby, on inflation. Thus, a stronger response of monetary policy to inflation ends up stabilizing inflation by less.

Moreover, Proposition 4 establishes that stronger the systematic response of monetary policy to inflation, greater will be the response of inflation to the non-policy shocks in equilibrium. This result is again in contrast to the result under the AMPF regime. What leads to this result? When a positive non-policy shock hits the economy, it raises inflation. Now with a higher $\phi$, interest rates will rise by more in response to this increase in inflation, as given by Equation (3). Under the AMPF policy regime, this increase in interest rates would bring expected inflation down. In this PMAF regime, however, the greater increase in interest rates raises the value of government debt by a greater amount. This leads to a greater wealth effect on the households, which increases inflation by a larger amount.

Finally, Proposition 4 shows that a greater systematic response of interest rates to inflation leads to a greater response of inflation to public debt outstanding. Again, this result arises because with a stronger response of interest rates to inflation, the wealth effect gets amplified. As shown in Proposition 3, a higher value of $\hat{b}_{t-1}$ increases inflation. While the monetary authority raises the interest rate in response to the increased inflation, the interest rate will rise by more with a higher $\phi$, which in turn will deliver a stronger wealth effect.

In this regime, since fiscal policy also matters for inflation dynamics, we next establish a result related to the fiscal policy stance.

Proposition 5 (Magnitude of inflation response and fiscal policy stance) Under PMAF, inflation deviates even further from the inflation target as the fiscal authority becomes more

\footnotetext{
${ }^{15}$ Again, note that for the first and the third result of Proposition 4 , there is a condition on $\psi$ such that fiscal policy has to be "sufficiently" active. That is, $\psi<\bar{\psi}^{* *}, \bar{\psi}^{* * *} \leq 1-\beta$. As we argue in the appendix, this condition is unlikely to be relevant empirically.
} 
active - that is, $\Phi$ increases as $\psi$ decreases in the domain of $(-\infty, 1-\beta)$ :

$$
\frac{\partial \Phi(\phi, \psi)}{\partial \psi}<0 \quad \text { for } \psi \in(-\infty, 1-\beta) \text { and } \phi \in[0,1)
$$

Moreover, inflation responds less in response to non-policy shocks as the fiscal authority becomes more active - that is, $\Gamma$ decreases as $\psi$ decreases in the domain of $(-\infty, 1-\beta)$ :

$$
\frac{\partial \Gamma(\phi, \psi)}{\partial \psi}>0 \quad \text { for } \psi \in(-\infty, 1-\beta) \text { and } \phi \in[0,1)
$$

Finally, inflation responds more to a change in public debt outstanding when the fiscal authority becomes more active - that is, $\Omega$ increases as $\psi$ decreases in the domain of $(-\infty, 1-\beta)$ :

$$
\frac{\partial \Omega(\phi, \psi)}{\partial \psi}<0 \quad \text { for } \psi \in(-\infty, 1-\beta) \text { and } \phi \in[0,1)
$$

In this PMAF regime, Proposition 5 shows that as fiscal policy becomes more active, inflation responds more strongly, and in the opposite direction, to changes in the inflation target. This result arises because as $\psi$ decreases, taxes respond less strongly to debt as given by Equation (4). Then the wealth effect due to interest rate changes described above becomes amplified. This increased wealth effect in turn leads to greater spending and thereby a stronger response of inflation.

Proposition 5 also shows that weaker is the response of taxes to debt, lower is the response of inflation to the non-policy shock. When a positive $\hat{r}_{t}^{*}$ shock hits the economy, as we discussed above, it leads to higher inflation. This lowers the value of government debt. From Equation (4), this implies that taxes will decrease. Now, lower is $\psi$, smaller is the decrease in taxes. Even though taxes are lump-sum in our model, when the regime is PMAF, tax changes lead to a wealth effect on households. With a smaller decrease in taxes, the wealth effect is smaller, which in turn leads to a smaller change in spending and thereby inflation. Finally, the last result in Proposition 5 shows that inflation responds more to a change in public debt outstanding when fiscal authority becomes more active arises because the wealth effect gets magnified when taxes respond less to public debt outstanding.

So far, we have provided analytical results in the PMAF regime for the initial response of inflation. Because of the role of an endogenous state variable, it is cumbersome to derive closed-form results for the full dynamic response of inflation. We therefore, resort to numerical solutions to show that our results on the initial response of inflation are general indications of the overall dynamic responses as well.

We show in Figure 2 the response of inflation to a one percent decrease in the inflation target under varying degrees of monetary and fiscal policy stances. The figure highlights our 
results above in Propositions 3, 4, and 5 and shows that while our analytics focused on the impact response of inflation, the same economic intuition can be extended to longer horizons. Indeed, it clearly shows that the deviation of inflation from the target continues to be greater in periods following the change in the inflation target, as monetary and fiscal policies become more active. ${ }^{16}$ The reason is that when $\phi$ is higher (and/or $\psi$ is lower), the interest rate will be persistently higher after a decrease in the inflation target to the extent that the inflation target is persistent. This leads to a persistently higher value of public debt, which in turn, leads to a persistently positive wealth effect and a persistently higher inflation. What is more, as monetary and/or fiscal policy is more active, inflation depends more strongly on government indebtedness - that is, as shown above in Propositions 4 and $5, \Omega(\phi, \psi)$ is increasing in $\phi$ and decreasing in $\psi$. This property magnifies the mechanism through which larger debt outstanding influences the dynamics of inflation.

Figure 3 illustrates our results on the inflation response to non-policy shocks under varying degrees of monetary and fiscal policy reactions to inflation and debt, respectively. The figure highlights our analytical results above on the impact response of inflation and the dynamic responses of inflation reveal an interesting pattern: while the initial response of inflation is positive in response to the shock $\hat{r}_{t}^{*}$ and it remains positive for a number of periods, after some time, inflation goes below steady state. ${ }^{17}$ The intuition for this result is again related to the dynamics of government debt. Initially, the increase in inflation lowers the value of government debt. In this regime, this decrease in the value of government debt leads to a negative wealth effect on households. This negative wealth effect leads to a decrease in spending by households, which eventually leads to inflation decreasing and going below steady state. Moreover, note that in Panel (a) of Figure 3 one sees that the paths intersect after a certain number of periods. This feature arises because when inflation goes below steady state, it leads to a decrease in nominal interest rates, as given by Equation (3). This decrease in interest rate leads to a negative wealth effect. Higher the value of $\phi$, greater is this negative wealth effect. Thus, once inflation goes below steady state, due to the negative wealth effect that depresses spending, there is a tendency for inflation to continue below steady for a while. This effect is more pronounced when $\phi$ is higher, which in turn, implies that the paths for different levels of $\phi$ will cross.

We next delve further into this issue. Figure 4 shows results for the inflation response

\footnotetext{
${ }^{16}$ Kim (2003) and Canzoneri, Cumby, and Diba (2011) present impulse response of inflation to a monetary shock under PMAF and show that a positive interest rate shock leads to an increase in inflation for several periods. Since a positive interest rate shock and a drop in the inflation target behave similarly, our results are consistent with theirs. They do not however, consider detailed comparative statics with respect to monetary and fiscal policy parameters.

${ }^{17} \mathrm{Kim}$ (2003) also present impulse response of inflation to aggregate demand and aggregate supply shocks under PMAF and notes this behavior of "inflation reversal." He does not however, consider detailed comparative statics with respect to monetary and fiscal policy parameters.
} 
to non-policy shocks under varying degrees of monetary policy reaction to inflation and for different levels of persistence of the non-policy shocks. As is to be expected, the greater the persistence of the shock, the more persistent will be the response of inflation. Moreover, the pattern of inflation initially remaining above steady state and then eventually going below steady state is robust to various levels of persistence of the shock.

Figure 5 illustrates our results on the effect of a change in public debt outstanding on inflation and how the effect varies with monetary and fiscal policy stances. ${ }^{18}$ We consider a one percent increase in debt outstanding and show that, as proved in Propositions 3, 4, and 5 , it leads to a positive initial response of inflation, the extent of which increases as monetary and fiscal policy become more active. Moreover, Figure 5 shows that the analytical results on the initial effect on inflation extend robustly to the dynamic response: the response of inflation continues to be greater in periods following a change in public debt outstanding, as monetary and fiscal policies become more active.

The results on the dynamic responses of inflation have an important implication for the relationship between the monetary policy stance and the volatility of inflation - a primary policy objective of central banks. As Figure 3 illustrates, under PMAF, while the response of inflation deviation is not greater for every time period when $\phi$ is higher, it is certainly the case for most periods - especially the initial periods. To the extent that initial responses of inflation to shocks dominate in the second moment of inflation dynamics, the volatility of inflation will be larger when monetary policy reaction is stronger. ${ }^{19}$ Figure 6 illustrates this result. Under AMPF, a more hawkish monetary policy leads to a smaller standard deviation of inflation as discussed earlier. Under PMAF, however, a stronger monetary policy reaction to inflation instead leads to a higher volatility of inflation.

Finally, we consider a regime where there is equilibrium indeterminacy as both monetary and fiscal policies are passive.

\subsubsection{Passive Monetary and Passive Fiscal Policy}

Under a passive monetary and passive fiscal (PMPF) policy regime, multiple equilibria exist. We can express the solution for inflation as:

$$
\hat{\pi}_{t}=-\kappa \beta^{-1} \tilde{Y}_{t-1}+\beta^{-1} \hat{\pi}_{t-1}-\Phi_{\pi}(\phi, \psi) \hat{\pi}_{t}^{*}-\Gamma_{\pi}(\phi, \psi) \hat{r}_{t}^{*}+\Lambda_{\pi}(\phi, \psi)\left(M_{\pi} \hat{\pi}_{t}^{*}+M_{r} \hat{r}_{t}^{*}+\zeta_{t}^{*}\right),
$$

\footnotetext{
${ }^{18}$ Obviously, what is needed to compute these figures in addition to the solution for inflation is the equilibrium law of motion of debt, the closed-form expression for which is provided in the appendix.

${ }^{19}$ Initial responses are disproportionately important for the variance of the inflation rate because the squared size of the initial response to a shock is substantially bigger than those of the responses in the following periods as can be seen in Figure 3. This argument is reminiscent of the difference in outcomes when monetary policy is analyzed under commitment and discretion, also known as the stabilization bias.
} 
where $\zeta_{t}^{*}$ is a sunspot shock that is independent of the fundamental shocks to the economy. Moreover, $M_{\pi}$ and $M_{r}$ are new parameters introduced due to indeterminacy that are not pinned down by the structural parameters of the model, while $\Phi(\phi, \psi)$ and $\Gamma(\phi, \psi)$ capture the part of the solution that is determined uniquely by the structural parameters. ${ }^{20}$

In this regime, we establish the following result where for analytical tractability, we focus on iid shocks.

Proposition 6 Suppose that $\rho_{\pi}=\rho_{r}=0$. Under PMPF

$$
\Phi(\phi, \psi) \geq 0 \text { and } \Gamma(\phi, \psi)>0 \quad \text { for } \psi \in(1-\beta, \infty) \text { and } \phi \in[0,1) \text {. }
$$

The equality holds when $\phi=0$. Moreover, $\hat{b}_{t-1}$ is not a state variable for inflation.

Proposition 6 thus shows that under PMPF, for the part of the solution that is determined by the structural parameters of the model, the initial response of inflation to $\hat{\pi}_{t}^{*}$ and $\hat{r}_{t}^{*}$ depends on both monetary and fiscal policy parameters. In this respect, the solution is similar to that under the PMAF regime and unlike that under the AMPF regime. Thus, under indeterminacy, not explicitly specifying fiscal policy in the model, even though it is passive, is a source of misspecification. Moreover, Proposition 6 establishes that $\hat{b}_{t-1}$ does not directly affect inflation dynamics under the PMPF regime. This is a critical difference from the PMAF regime. Therefore, while indeterminacy changes the propagation mechanism of fundamental shocks compared to AMPF and PMAF, it does not introduce public debt as a state variable unlike PMAF since fiscal policy is passive. Thus, fiscal policy affects the equilibrium law of motion of inflation in a subtle but important way under PMPF, implying that it cannot be left unspecified in the model.

Finally, for the part of the solution that is determined by the structural parameters of the model, inflation moves in the opposite direction from a change in the inflation target. ${ }^{21}$

\footnotetext{
${ }^{20}$ For the complete description of the notation, see the appendix. Our solution methodology follows Lubik and Schorfheide (2003). The assumption that $\zeta_{t}^{*}$ is independent of the fundamental shocks is not consequential for the dynamics of inflation. The last term in (10) appearing due to indeterminacy, $M_{\pi} \hat{\pi}_{t}^{*}+M_{r} \hat{r}_{t}^{*}+\zeta_{t}^{*}$, can be written as a single composite non-fundamental shock that may be correlated with the fundamental shocks to any extent. Let us denote the non-fundamental shock by $\tilde{\zeta}_{t}^{*}$. Then, there are three parameters for the distribution of $\tilde{\zeta}_{t}^{*}$ - the covariance with $\hat{\pi}_{t}^{*}$ and $\hat{r}_{t}^{*}$, and the variance - for which there exists a one-to-one mapping with $M_{\pi}, M_{r}$, and the variance of $\zeta_{t}^{*}$. That is, one can always find a unique non-fundamental shock that is in general correlated with the fundamental shocks and implies equivalent dynamics for inflation.

${ }^{21}$ Lubik and Schorfheide (2003) show in a three-equation model without fiscal policy and variables that for the part of the solution determined by structural parameters of the model, the initial effect of a positive monetary shock on inflation is positive. Our results are thus consistent with theirs, since a positive monetary shock and a negative inflation target shock behave similarly, and moreover, provide a generalization since we consider a model with fiscal policy. In addition, as we note above, not including fiscal policy explicitly under indeterminacy is a source of misspecification.
} 
This is again similar to the case under the PMAF regime. Unlike the case under PMAF (and AMPF) however, the initial effect of the shock $\hat{r}_{t}^{*}$ on inflation is negative.

Importantly, note that the overall relationship between inflation and the inflation target and the total initial effect of $\hat{r}_{t}^{*}$ on inflation is ambiguous, depending on the values taken on by $M_{\pi}$ and $M_{r}$. If agents form self-fulfilling expectations that an increase in the inflation target pushes up inflation significantly $\left(\Lambda(\phi, \psi) M_{\pi}>\Phi(\phi, \psi)\right)$, then inflation indeed responds positively to the increase of the inflation target. Therefore, in general, the question of how inflation is related to the inflation target (and how inflation responds to $\hat{r}_{t}^{*}$ ) can be answered only empirically as it will depend on the estimates of both non-structural and structural parameters. For example, in an estimated model of the US economy in the pre-Volcker era, Bhattarai, Lee, and Park (2012b) find that an increase of the inflation target decreases inflation, as depicted in Figure 1.

\subsection{Comparison with Unpleasant Monetarist Arithmetic}

So far, we have shown that one of the major differences between an AMPF and a PMAF policy regime is in the response of inflation to a change in the inflation target and public debt outstanding. A classic model provided by Sargent and Wallace (1981), where the central bank monetizes government debt as it is under "fiscal dominance," is another example in the literature where government debt can influence inflation dynamics. In this section we illustrate the main mechanism behind this well-known "Unpleasant Monetarist Arithmetic" result using an extension of our model and analyze how some properties of the PMAF regime differ from that of a fiscal dominance regime in the spirit of Sargent and Wallace (1981). ${ }^{22}$

To illustrate the unpleasant monetarist arithmetic mechanism, we make some minimal changes to our model. In particular, since the mechanism requires the central bank to raise seigniorage revenues to back up government debt when taxes do not respond strongly enough to debt, we introduce money by using the money-in-the-utility specification. This leads to a standard money demand function, $\hat{m}_{t}=\eta_{y} \hat{Y}_{t}-\eta_{i} \hat{R}_{t}$, where $\hat{m}_{t}$ is real balances and $\eta_{y}$ and $\eta_{i}$ are (semi-)elasticity of money demand, and extends the government budget constraint to include the revenue raised by money creation. In addition, rather than an interest-rate rule, we now model monetary policy using a seigniorage rule in the tradition of Sargent and Wallace (1981). ${ }^{23}$ Specifically, the government budget constraint and seigniorage-based

\footnotetext{
${ }^{22}$ Canzoneri, Cumby, and Diba (2011) is a recent comparison of these two mechanisms. Our relative contribution is to explain these issues in a unified framework of a sticky price model with policy rules and a focus on dynamics.

${ }^{23}$ Fiscal policy also follows a tax-based rule. Thus we do not consider a game between the monetary and fiscal authority.
} 
monetary policy rule are given respectively as:

$$
\begin{aligned}
& \hat{b}_{t}=\beta^{-1} \hat{b}_{t-1}-\beta^{-1} \bar{b} \hat{\pi}_{t}-\beta^{-1} \hat{\tau}_{t}-\beta^{-1} \hat{s}_{t}+\bar{b} \hat{R}_{t}, \\
& \hat{s}_{t}=\xi_{b} \hat{b}_{t-1}+\varepsilon_{s, t},
\end{aligned}
$$

where $\hat{s}_{t} \equiv \bar{m}\left(\hat{m}_{t}-\hat{m}_{t-1}+\hat{\pi}_{t}\right)$ is seigniorage and $\varepsilon_{s, t}$ is a shock. The central bank may adjust seigniorage in response to changes in government debt outstanding if $\xi_{b} \neq 0$. Finally, Equations (1) and (2), as well as the fiscal policy rule (4), continue to apply in this model. ${ }^{24}$

As we have discussed before, the PMAF policy regime operates through changes in household spending arising from wealth effects that are generated because the government budget constraint is not satisfied at prevailing prices. As a result of these spending changes, inflation evolves and revalues government debt to ensure that the government budget constraint is satisfied in equilibrium. On the other hand, the fiscal dominance regime of this section operates through revenue generation by money creation. To make this distinction stark, we now assume that $\bar{b}=0$, which simplifies the government budget constraint to:

$$
\hat{b}_{t}=\beta^{-1} \hat{b}_{t-1}-\beta^{-1} \hat{\tau}_{t}-\beta^{-1} \hat{s}_{t} .
$$

Now, there simply is no possibility of inflation revaluing debt to lead to stable dynamics as inflation does not affect debt dynamics at all. ${ }^{25}$ Therefore, the only way in which debt dynamics can be stable even when taxes do not respond strongly enough to debt is if seigniorage revenues $\hat{s}_{t}$ adjust. That is, the central bank has to monetize government debt.

We now define two regimes that lead to a unique stable equilibrium: a "monetary dominance" regime and a "fiscal dominance" regime. In the monetary dominance regime, monetary policy sets an exogenous stochastic path for seigniorage without concerning itself with debt stabilization $\left(\xi_{b}=0\right)$, while fiscal policy adjusts taxes by enough to stabilize debt (a high enough $\psi$ ). In the fiscal dominance regime, monetary policy has to adjust seigniorage revenue to adjust for debt (a positive and large enough $\xi_{b}$ ) since fiscal policy does not adjust taxes by enough to stabilize debt (a low $\psi$ ). The exact bounds on $\xi_{b}$ and $\psi$ that characterize these regimes are: monetary dominance by $\xi_{b}=0$ and $\psi>1-\beta$, and fiscal dominance by $\xi_{b}>0$ and $\psi<1-\beta .{ }^{26}$ Note that if seigniorage and tax revenues jointly do not adjust by enough to back up debt $\left(\xi_{b}+\psi<1-\beta\right)$, then a stable equilibrium does not exist. Finally, unlike before, there is no possibility of indeterminacy of equilibria as with a seigniorage/money supply based

\footnotetext{
${ }^{24}$ For concreteness of comparison, we abstract from non-policy shocks in this section. Thus, $\hat{Y}_{t}=\tilde{Y}_{t}$.

${ }^{25}$ Another way to make the same point would be to have real government bonds, as in Sargent and Wallace (1981), instead of nominal government bonds.

${ }^{26}$ The detailed derivations are in the appendix. Also, for fiscal dominance to lead to stable dynamics, we need to have $\xi_{b}+\psi>1-\beta$.
} 
monetary policy rule. ${ }^{27}$

In the appendix, we prove the following result.

Proposition 7 Under monetary dominance, $\hat{b}_{t-1}$ is not a state variable of the model and $\hat{\pi}_{t}$ evolves independently of public debt dynamics. Under fiscal dominance, $\hat{b}_{t-1}$ is a state variable of the model.

It is relatively straightforward to understand the intuition behind Proposition 7. Under monetary dominance, the model becomes block-recursive where the tax rule (4) and Equation (11) determine the (stable) dynamics of $\hat{b}_{t}$ and $\hat{\tau}_{t}$ independently of other model variables. Under fiscal dominance, as is clear from the system of equilibrium conditions, $\hat{b}_{t-1}$ is a state variable as seigniorage revenues have to adjust to back up (part of) public debt. In this sense, the fiscal dominance regime is similar to the PMAF regime.

We next show how inflation evolves when the model is perturbed by the seigniorage shock $\varepsilon_{s, t}$ and a change in outstanding government debt. We rely on numerical results as the model is cumbersome to solve in closed-form. ${ }^{28}$ Panel (a) of Figure 7 shows that in response to a positive seigniorage shock, inflation increases under both the monetary and fiscal dominance regimes. This result is robust to a wide range of realistic parameter values and simply reflects the fact that money creation is inflationary. In particular this result illustrates a difference between the fiscal dominance regime and the PMAF regime. Recall that in Proposition 3 we had shown that the effect of an increase in the inflation target was in the opposite direction under the PMAF regime as compared to the AMPF regime. This is not the case here. Moreover, while considering the dynamics and comparing Panel (a) with Panel (b) of Figure 7 , which plots the response of seigniorage to the shock, it is clear that regardless of the regime, inflation is a monetary phenomenon. This is also different from the comparison between the AMPF and PMAF regimes. At the same time however, note that after some periods, inflation goes below steady state for several periods under fiscal dominance but not under monetary dominance. This result is in the spirit of Sargent and Wallace (1981) since it shows that under fiscal dominance, the central bank eventually has to reduce seigniorage and decrease inflation after expanding seigniorage currently. Thus, in this sense there is a similarity with PMAF in that the central bank has imperfect control over inflation dynamics.

\footnotetext{
${ }^{27}$ Sargent and Wallace (1981) make a related point in their classic study as well.

${ }^{28}$ We use a standard calibration of $\beta=0.99$ and $\kappa=0.179$. For the elasticity parameters of the money demand function, we use values similar to the estimates in Ireland (2004): $\eta_{y}=0.014$ and $\eta_{i}=1.386$. We use $\bar{m}=1$, which does not affect model dynamics qualitatively. For policy parameters, we set $\xi_{b}=0$ and $\psi=0.1$ for the monetary dominance case and $\xi_{b}=0.1$ and $\psi=0$ for the fiscal dominance case. The shock to the seignorage follows an $\mathrm{AR}(1)$ process with $\mathrm{AR}$ coefficient 0.995 while a change in government debt outstanding is one-time only.
} 
Panel (c) of Figure 7 shows that in response to an increase in public debt outstanding, inflation increases under fiscal dominance while it is not affected at all under monetary dominance. This is indeed one implication of Proposition 7 since under monetary dominance, public debt is not a state variable. Since in this regime taxes do not fully back debt, when public debt outstanding increases, seigniorage revenues have to be raised to avoid explosive debt dynamics and fulfill the government budget constraint. Increase in seigniorage then generates inflation. Thus, with respect to the implication of government debt outstanding for inflation dynamics, the fiscal dominance regime is similar to the PMAF regime.

We have thus shown that in some respects the fiscal dominance regime of Sargent and Wallace (1981) is similar to the PMAF regime. In particular, under both regimes, government debt affects inflation dynamics and an increase in government debt outstanding is inflationary. It is important to note however, that the mechanisms that lead to this similar result are different. In fact we have illustrated them in model environments where we have shut down one mechanism at a time. We presented the PMAF regime in a model without money, where the possibility of revenues from money creation backing debt never arises. The channel that leads to the government budget constraint being satisfied even with a low response of taxes to debt is then revaluation of nominal debt by inflation. We then presented the fiscal dominance regime in a model where the possibility of revaluation of nominal debt never arises because inflation does not affect (up to linear approximation) debt dynamics at all. The channel that leads to the government budget constraint being satisfied even with a low response of taxes to debt is then revenue generation through printing of money.

\section{Quantitative Model}

In this section, we assess whether the results found analytically with the simple model also hold in a quantitative model that features a rich set of frictions and shocks along the lines of Smets and Wouters (2007). In particular, our model, other than a slightly different specification of monetary policy rule and an inclusion of a fiscal block, is very similar to Del Negro, Schorfheide, Smets, and Wouters (2007) and Justiniano, Primiceri, and Tambalotti (2010). ${ }^{29}$

For our numerical exercise, we use the posterior median estimates of Justiniano, Primiceri, and Tambalotti (2010) for all the parameters related to preferences and technology. For all three policy regimes, we also use the same value as their posterior median estimates for the

\footnotetext{
${ }^{29}$ The main differences in the monetary policy rule specification is that we include a time-varying inflation target while excluding the growth rate of the output gap. Fiscal policy on the other hand is modelled using a tax rule that features tax smoothing and a systematic response of the tax revenues to the level of outstanding government debt as in (4). In addition, the government spending-to-output ratio evolves exogenously as a time-varying fraction of output. As before, we obtain a first-order approximation of the equilibrium conditions around a steady state (after normalization since there is growth) and solve using standard methods.
} 
monetary policy feedback parameter on output gap. For the tax smoothing parameter in the fiscal policy rule, we use the posterior estimate of Bhattarai, Lee, and Park (2012b), while for the steady state level of the maturity value of debt-to-output, we use the sample average from U.S. data. We then conduct several comparative static exercises with respect to the policy feedback parameters on inflation and government debt outstanding. ${ }^{30}$ We provide the details of the numerical exercise with a complete model description in the appendix.

The numerical results from this quantitative model are consistent with our analytical results from the simple model. We present impulse response functions to a change in the inflation target and public debt outstanding. For non-policy shocks, to preserve space, we only present results on the volatility of inflation. ${ }^{31}$

\subsection{A Change in the Inflation Target}

Panels (a)-(d) of Figure 8 show for the three policy regimes the impulse response of inflation to an unanticipated change in the inflation target, varying the degree of monetary policy stance. They clearly illustrate one of the main results of our paper: under AMPF, inflation moves in the same direction as the inflation target and that higher the systematic response of monetary policy to inflation, smaller is the gap between inflation and the inflation target; while under PMAF, in sharp contrast, inflation moves in an opposite direction from the inflation target and higher is the systematic response of monetary policy to inflation, larger is the gap between inflation and the inflation target. Moreover, under PMPF, depending on the value of the parameter $M_{\pi}$, which governs how self-fulfilling beliefs are formed under indeterminacy, inflation could either move in the same direction as the inflation target or in an opposite direction. Finally, under PMAF, Panel (e) of Figure 8 shows that lower is the response of taxes to debt, greater is the gap between inflation and the inflation target.

\subsection{A Change in Public Debt Outstanding}

Similar to the simple model, under AMPF and PMPF, a change in public debt outstanding does not affect inflation in equilibrium as public debt outstanding is not a state variable. Figure 9 therefore shows results only for the PMAF policy regime. Consistent with the analytical result for the simple model, we find that inflation increases when the level of public debt

\footnotetext{
${ }^{30} \mathrm{As}$ in the simple model, the existence and uniqueness of equilibrium depends on the prevailing monetary and fiscal policy regime. In this richer model, since we are unable to analytically characterize the exact parameter boundaries that lead to various regimes, we determine them numerically.

${ }^{31}$ We have six non-policy shocks: neutral technology shock, government spending, investment specific technology shock, price markup shock, wage markup shock, and a preference shock. Even for impulse responses, our numerical results are consistent with the analytical model except for two cases of differences with respect to the initial impact on inflation. We provide all the figures as well as detailed explanations in the appendix.
} 
outstanding increases and that moreover, the response of inflation is higher when monetary policy responds more strongly to inflation and when taxes respond less strongly to public debt outstanding.

\subsection{Non-policy Shocks}

We now present the volatility of inflation in the presence of six non-policy shocks. This is especially pertinent because arguably, focusing on the volatility of inflation is the most sensible metric for inflation dynamics in this model which features various adjustment costs and internal propagation mechanisms. Figure 10 shows under the three policy regimes the standard deviation of inflation, varying the degree of monetary policy stance. Panels (a) and (b) clearly depict one of the main results of our paper: in response to non-policy shocks under AMPF, inflation volatility decreases as monetary policy responds strongly to inflation, while in sharp contrast, under PMAF, inflation volatility increases. In this particular parameterization, Panel (c) shows that under PMPF, inflation volatility increases as monetary policy responds more strongly to inflation.

\section{Conclusion}

In this paper we characterize the dynamics of inflation under different monetary and fiscal policy combinations in a standard DSGE model. First, using a simple set-up that allows for closed-form solutions, we show that answers to some classic questions in monetary economics, such as whether monetary policy controls inflation fully, whether higher level of government debt is inflationary, and how changes in monetary and fiscal policy stances affect inflation, depend crucially on the prevailing policy regime. Second, we show that our insights continue to hold in a richer quantitative model.

\section{References}

[1] Bhattarai, Saroj, Jae W. Lee, and Woong Y. Park. 2012a. "Monetary-Fiscal Policy Interactions and Indeterminacy in Post-War U.S. Data." American Economic Review, 102(3): 173-178.

[2] Bhattarai, Saroj, Jae W. Lee, and Woong Y. Park. 2012b. "Policy Regimes, Policy Shifts, and U.S. Business Cycles." Unpublished.

[3] Bianchi, Francesco and Cosmin Ilut. 2012. "Monetary/Fiscal Policy Mix and Agents' Beliefs." Unpublished. 
[4] Canzoneri, Matthew, Robert Cumby, and Behzad Diba. 2011. "The Interaction Between Monetary and Fiscal Policy." In Handbook of Monetary Economics, Vol. 3B, ed. Benjamin M. Friedman and Michael Woodford, 935-99. Amsterdam: Elsevier Science, NorthHolland.

[5] Clarida, Richard, Jordi Galí, and Mark Gertler. 2000. "Monetary Policy Rules and Macroeconomic Stability: Evidence And Some Theory." Quarterly Journal of Economics, 115(1): 147-180.

[6] Cogley, Timothy, Giorgio E. Primiceri, and Thomas J. Sargent. 2010. "Inflation-Gap Persistence in the U.S." American Economic Journal: Macroeconomics, 2(1): 43-69.

[7] Cogley, Timothy and Argia M. Sbordone. 2008. "Trend Inflation, Indexation, and Inflation Persistence in the New Keynesian Phillips Curve." American Economic Review, 98(5): 2101-26.

[8] Curdia, Vasco, Andrea Ferrero, Ging Cee Ng, and Andrea Tambalotti. 2011. "Evaluating Interest Rate Rules in an Estimated DSGE Model." Unpublished.

[9] Del Negro, Marco, Frank Schorfheide, Frank Smets, and Raf Wouters. 2007. "On the Fit and Forecasting Performance of New Keynesian Models." Journal of Business and Economic Statistics, 25(2): 123-162.

[10] Ireland, Peter N. 2007. "Changes in the Federal Reserve's Inflation Target: Causes and Consequences." Journal of Money, Credit and Banking, 39(8): 1851-1882.

[11] Justiniano, Alejandro, Giorgio E. Primiceri, and Andrea Tambalotti. 2010. "Investment Shocks and Business Cycles." Journal of Monetary Economics, 57(2): 132-145.

[12] Kim, Soyoung. 2003. "Structural Shocks and the Fiscal Theory of the Price Level in the Sticky Price Model." Macroeconomic Dynamics, 7: 759-782.

[13] Leeper, Eric M. 1991. "Equilibria under 'Active' and 'Passive' Monetary and Fiscal Policies." Journal of Monetary Economics, 27(1): 129-47.

[14] Loyo, Eduardo. 1999. "Tight Money Paradox on the Loose: A Fiscalist Hyperinflation." Unpublished.

[15] Lubik, Thomas A. and Frank Schorfheide. 2003. "Computing Sunspot Equilibria in Linear Rational Expectations Models." Journal of Economic Dynamics and Control, 28(1): 273285. 
[16] Lubik, Thomas A. and Frank Schorfheide. 2004. "Testing for Indeterminacy: An Application to U.S. Monetary Policy." American Economic Review, 94(1): 190-217.

[17] Primiceri, Giorgio E. 2006. "Why Inflation Rose and Fell: Policy-makers Beliefs and U.S. Postwar Stabilization Policy." Quarterly Journal of Economics, 121(3): 867-901.

[18] Sargent, Thomas J. and Neil Wallace. 1981. "Some Unpleasant Monetarist Arithmetic." Federal Reserve Bank of Minneapolis Quarterly Review, 5(3): 1-17.

[19] Sargent, Thomas J., Noah Williams, and Tao Zha. 2006. "Shocks and Government Beliefs: The Rise and Fall of American Inflation." American Economic Review, 96(4), 1193-1224.

[20] Sims, Christopher A. 1994. "A Simple Model for Study of the Determination of the Price Level and the Interaction of Monetary and Fiscal Policy." Economic Theory, 4(3): 381-99.

[21] Sims, Christopher A. 2004. "Limits to Inflation Targeting." In The Inflation Targeting Debate, ed. Ben S. Bernanke and Michael Woodford, 283-309. Chicago: University of Chicago Press.

[22] Sims, Christopher A. and Tao Zha. 2006. "Were There Regime Switches in U.S. Monetary Policy?." American Economic Review, 96(1): 54-81.

[23] Sims, Christopher A. 2011. "Stepping on a Rake: The Role of Fiscal Policy in the Inflation of the 1970s." European Economic Review, 55: 48-56.

[24] Smets, Frank and Raf Wouters. 2007. "Shocks and Frictions in U. S. Business Cycles: A Bayesian Approach." American Economic Review, 97(3): 586-606.

[25] Woodford, Michael. 1995. "Price-level Determinacy Without Control of a Monetary Aggregate." Carnegie-Rochester Conference Series on Public Policy, 43(1): 1-46.

[26] Woodford, Michael. 2003. Interest and Prices. Princeton, NJ: Princeton University Press. 


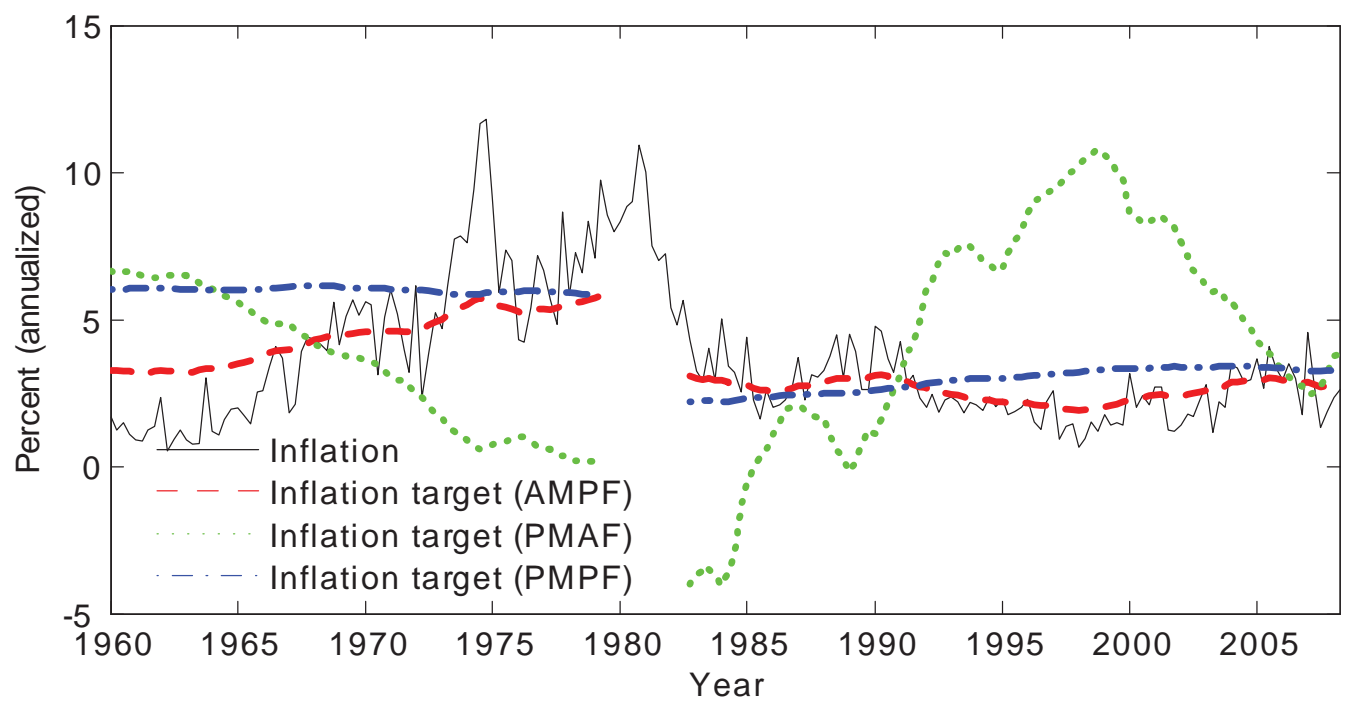

Figure 1: Inflation vs. estimated inflation target under different policy regimes in an estimated model using U.S. data.

Notes: The estimated inflation target is the point-wise posterior mean of the inflation target under different policy regimes in an estimated model using U.S. data in Bhattarai, Lee, and Park (2012b), from which the figure is reproduced. 
(a) Different values of $\phi$

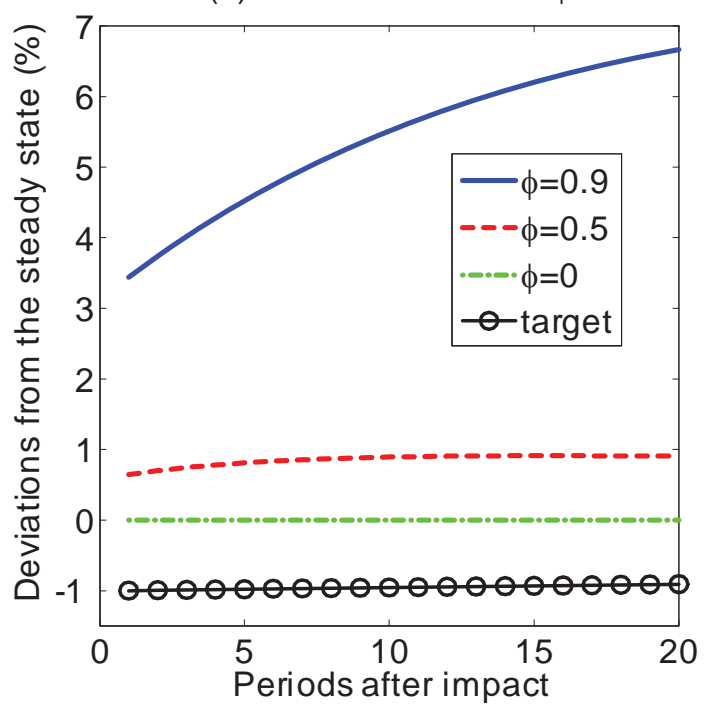

(b) Different values of $\psi$

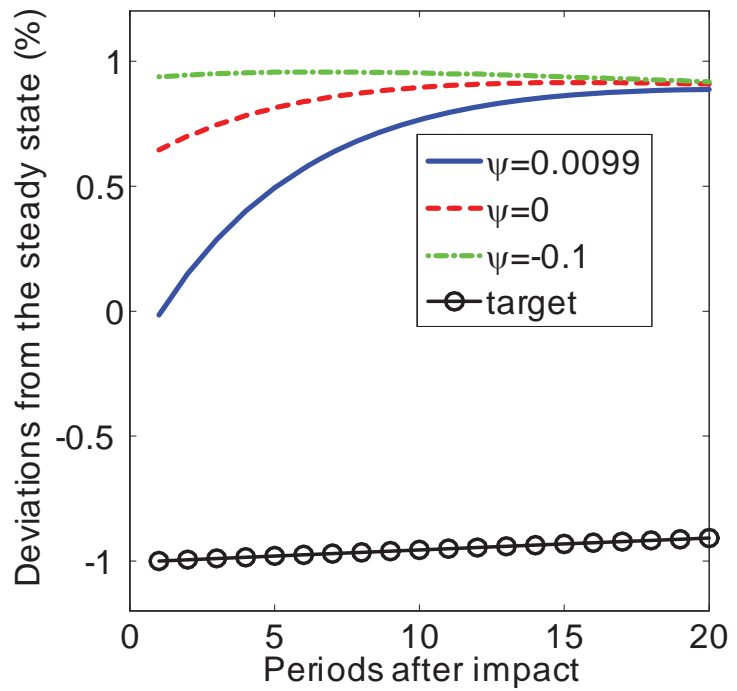

Figure 2: The response of inflation to a one percent decrease in the inflation target under the PMAF regime. 

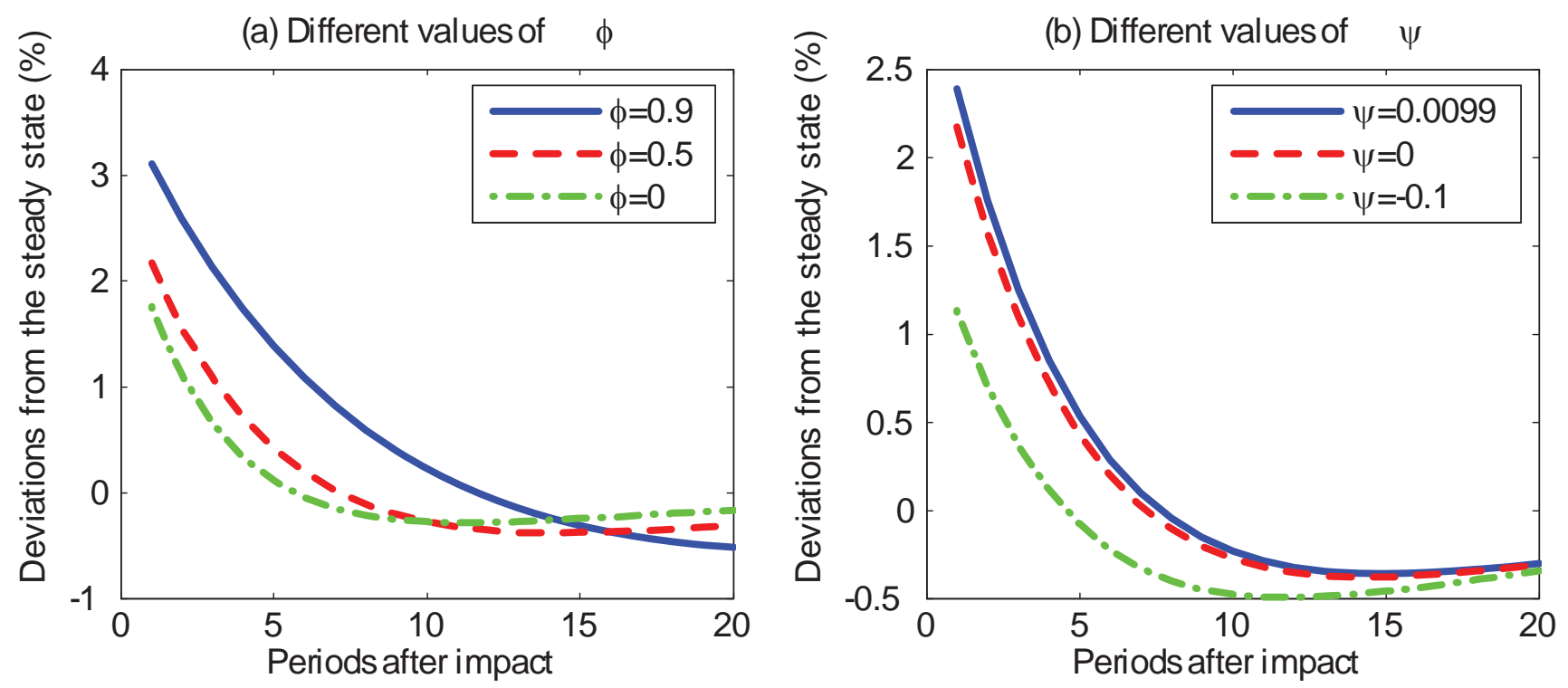

Figure 3: The response of inflation to a one percent increase in the non-policy shock under the PMAF regime. 
(a) $\rho_{r}=0.9$

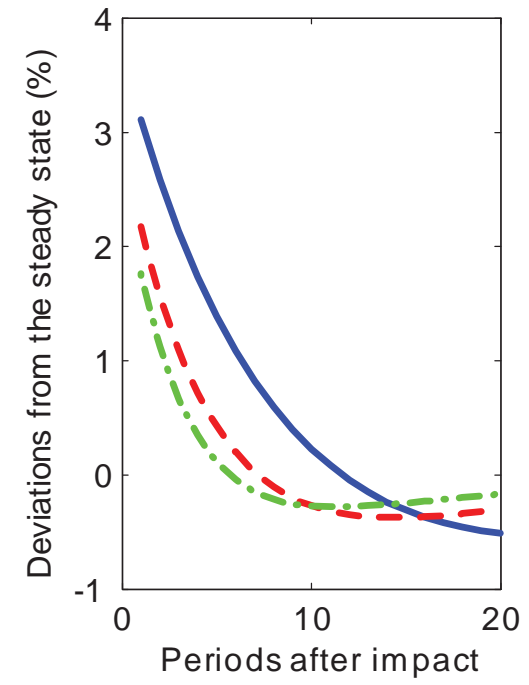

(b) $\rho_{r}=0.5$

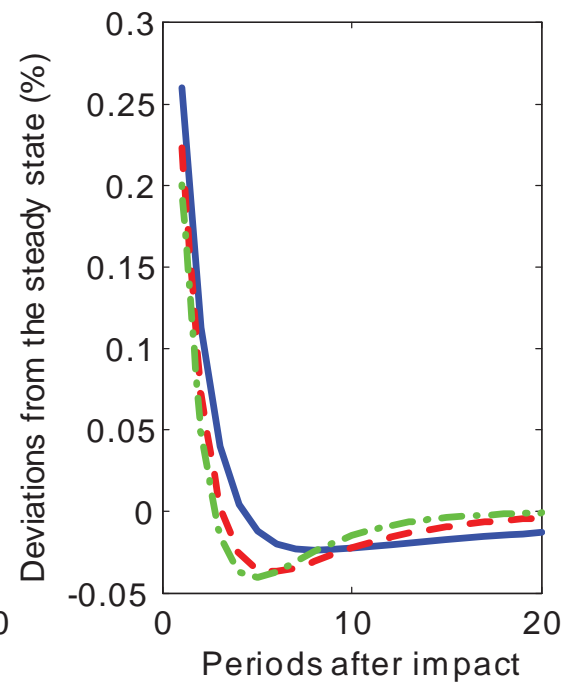

(c) $\rho_{\mathrm{r}}=0$

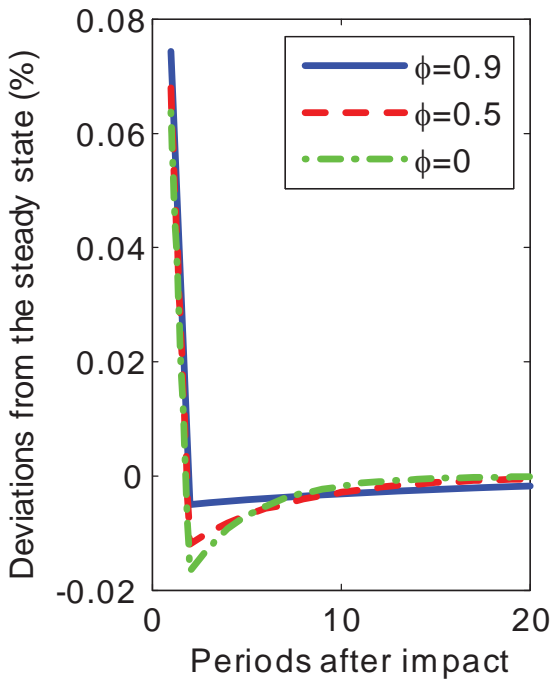

Figure 4: The response of inflation to a one percent increase in the non-policy shock with different degrees of persistence under the PMAF regime. 
(a) Different values of $\phi$

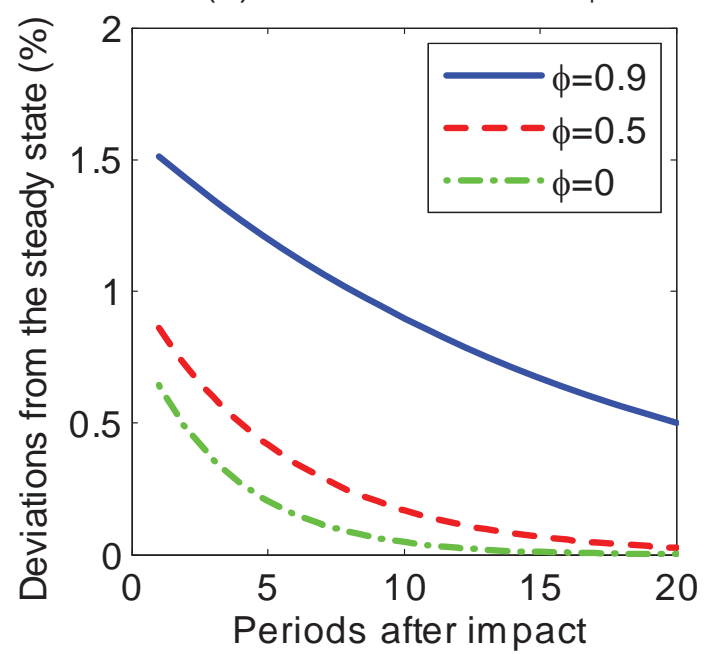

(b) Different values of $\psi$

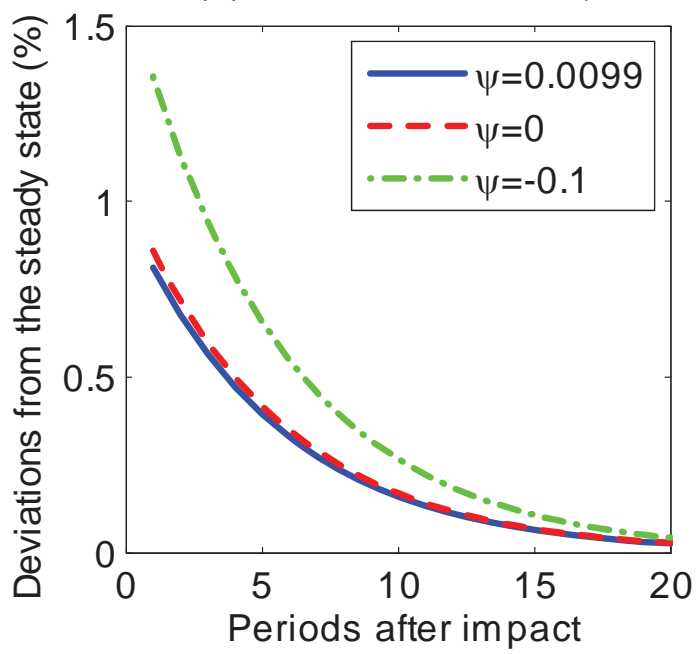

Figure 5: The response of inflation to a one percent increase in the level of debt-outstanding under the PMAF regime. 

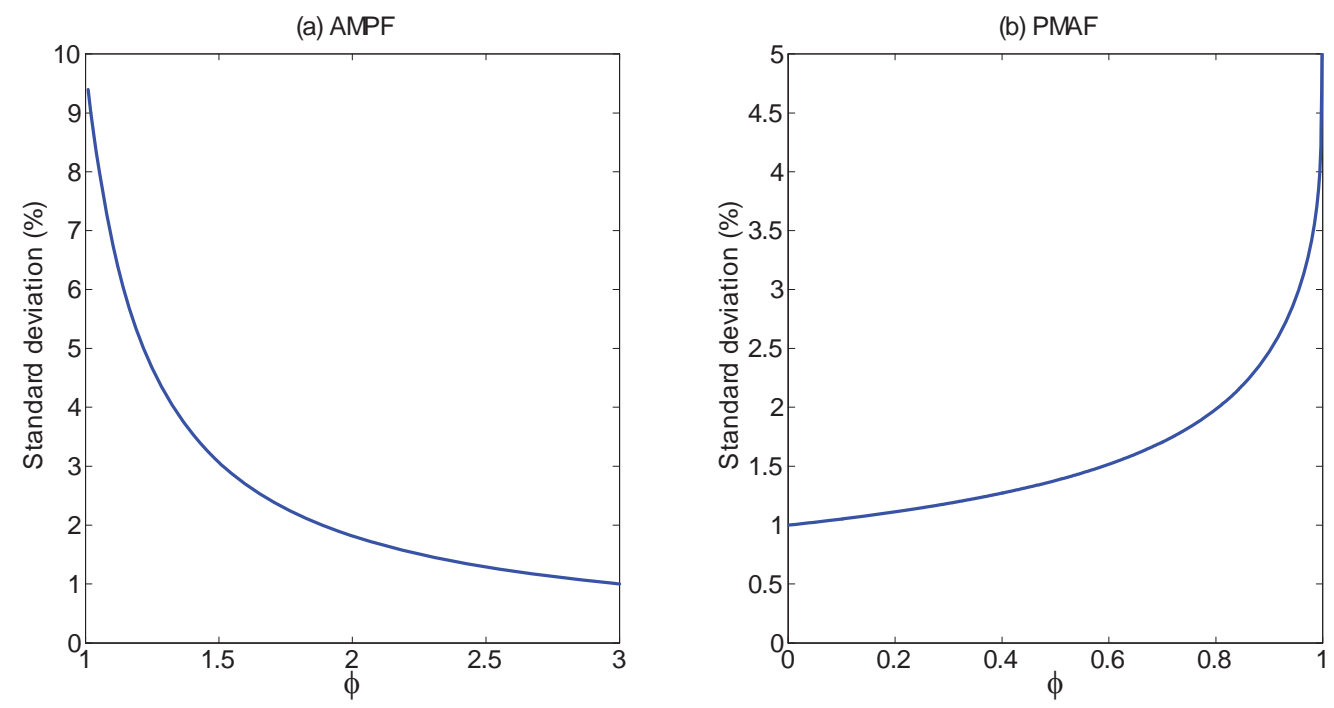

Figure 6: Standard deviation of inflation across different values of $\phi$ conditional on the nonpolicy shocks.

Notes: The standard deviation is rescaled so that it is equal to 1 when $\phi=3$ under AMPF and $\phi=0$ under PMAF. 

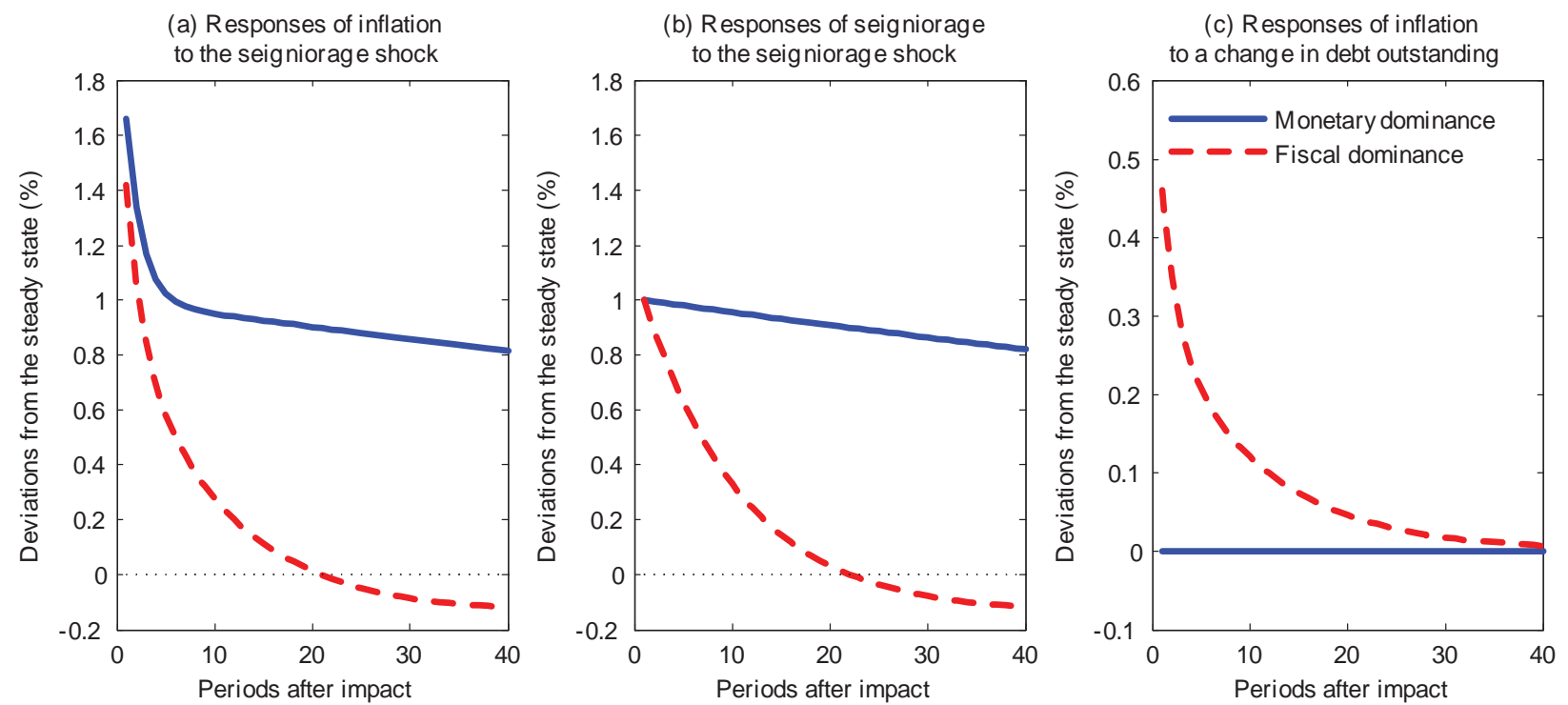

Figure 7: The impact of a one percent increase in the seigniorage shock and a one percent increase in debt outstanding in the unpleasant monetarist arithmetic model in the spirit of Sargent and Wallace (1981). 

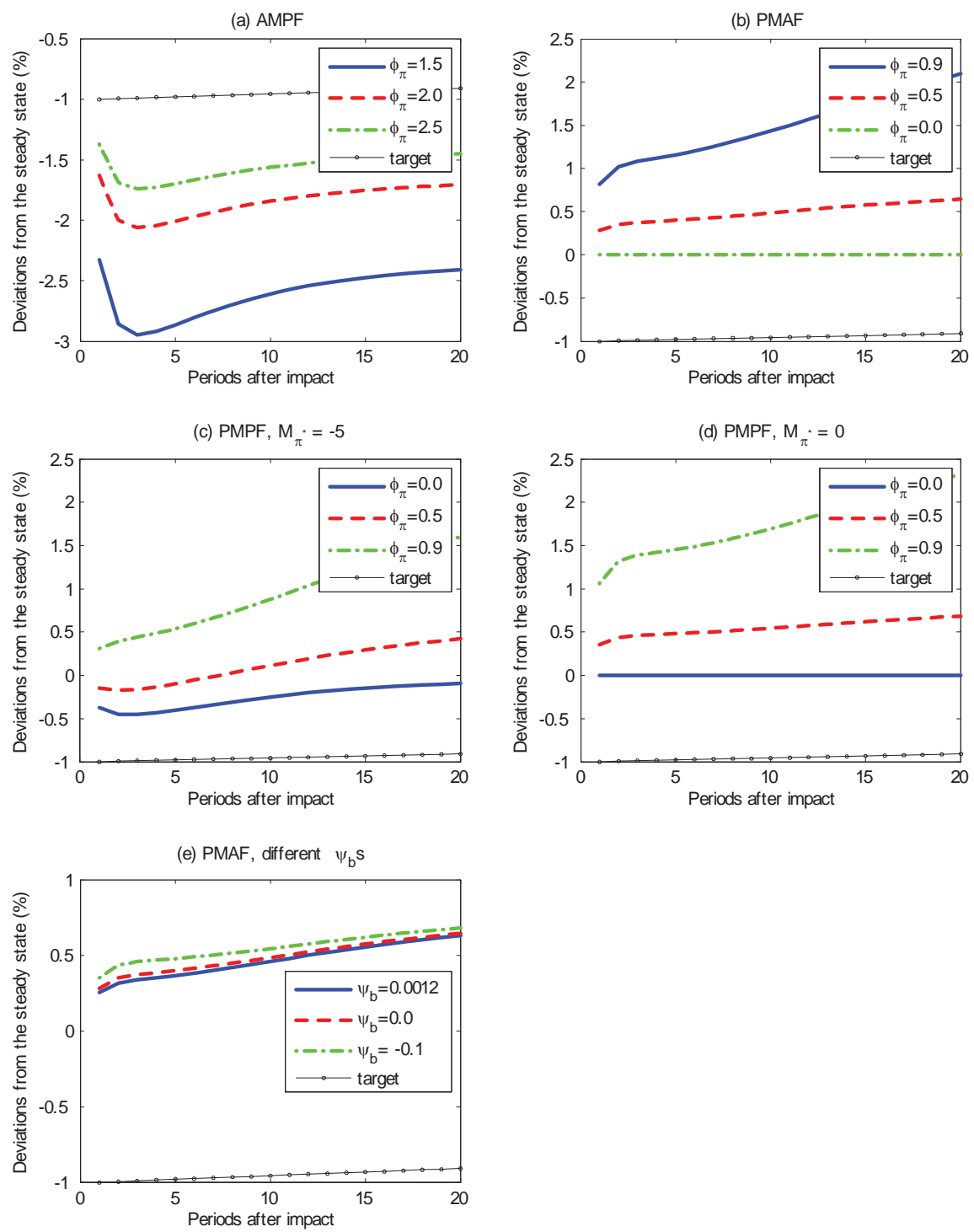

Figure 8: The response of inflation to a one percent decrease in the inflation target in the quantitative model. 
(a) PMAF, differen $\phi_{\pi} \mathrm{s}$

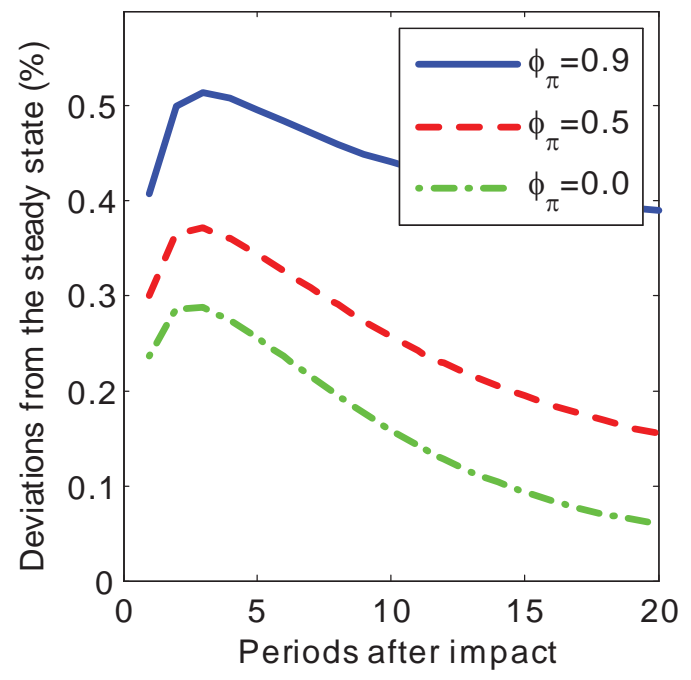

(b) PMAF, different $\psi_{b} s$

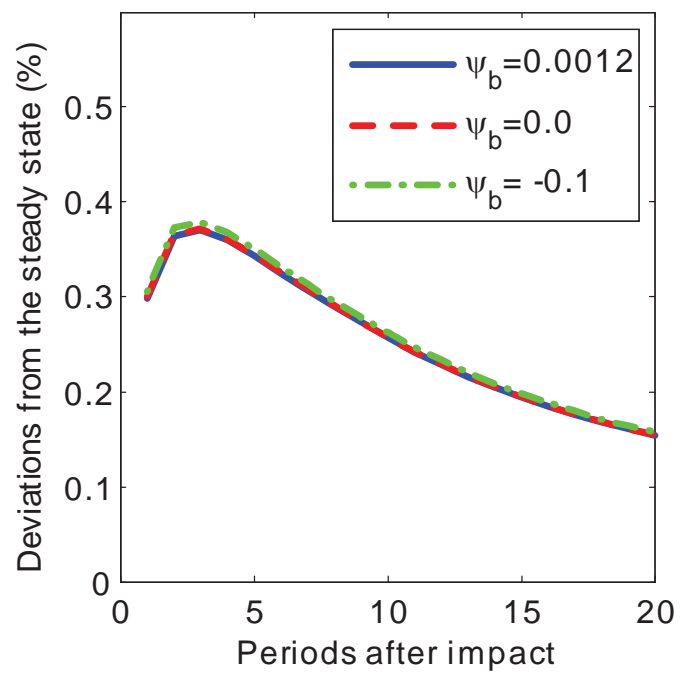

Figure 9: The response of inflation to a one percent increase in debt outstanding in the PMAF regime in the quantitative model. 
(a) AMPF

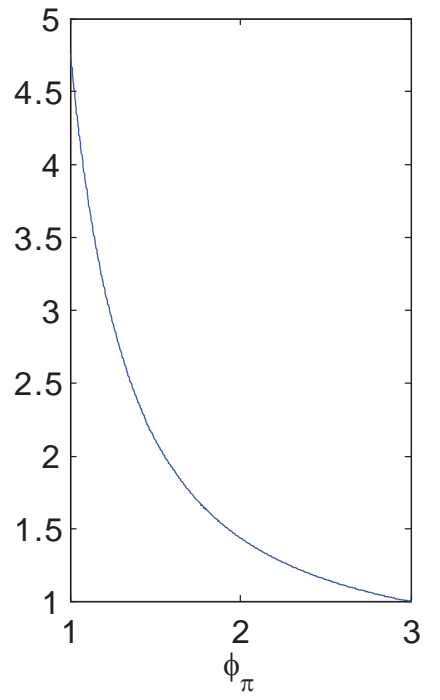

(b) PMAF

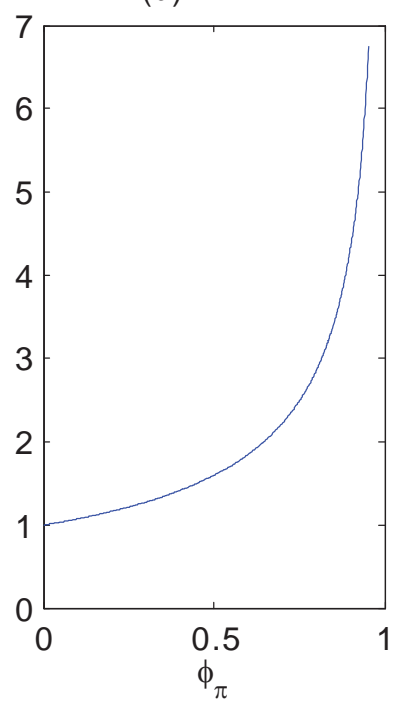

(c) PMPF

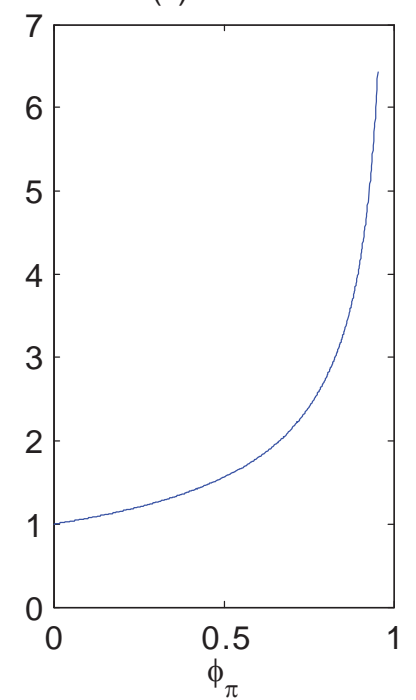

Figure 10: Standard deviation of inflation across different values of $\phi_{\pi}$ conditional on the non-policy shocks in the quantitative model.

Notes: The standard deviation is rescaled so that it is equal to 1 when $\phi=3$ under AMPF and $\phi=0$ under PMAF and PMPF. The self-fulfilling beliefs and the sunspot shock are turned off under PMPF. 


\section{Appendix}

\section{A Simple Model}

\section{A.1 Households}

Identical households choose sequences of $\left\{C_{t}, B_{t}, N_{t}, D_{t+1}\right\}$ to solve:

$$
\max E_{0} \sum_{t=0}^{\infty} \beta^{t} d_{t}\left[\log C_{t}-\frac{N_{t}^{1+\varphi}}{1+\varphi}\right]
$$

subject to

$$
P_{t} C_{t}+B_{t}+E_{t}\left[Q_{t, t+1} D_{t+1}\right]=R_{t-1} B_{t-1}+D_{t}+W_{t} N_{t}+\Pi_{t}-P_{t} \tau_{t}
$$

where $C_{t}$ is consumption, $N_{t}$ is labor hours, $P_{t}$ is the price level, $B_{t}$ is the amount of one-period risk-less nominal government bond, $R_{t}$ is the gross nominal interest rate, $W_{t}$ is the nominal wage rate, $\Pi_{t}$ is profits of intermediate firms, and $\tau_{t}$ is government taxes net of transfers. The parameter, $\varphi \geq 0$, denotes the inverse of the Frisch elasticity of labor supply, while $d_{t}$ represents an intertemporal preference shock. In addition to the government bond, households trade at time $t$ one-period state-contingent nominal securities $D_{t+1}$ at price $Q_{t, t+1}$.

\section{A.2 Firms}

Perfectly competitive firms produce the final good, $Y_{t}$, by assembling intermediate goods, $Y_{t}(i)$, through a constant-elasticity-of-substitution (CES) technology

$$
Y_{t}=\left(\int_{0}^{1} Y_{t}(i)^{\frac{\theta-1}{\theta}} d i\right)^{\frac{\theta}{\theta-1}}
$$

where $\theta>1$ denotes the elasticity of substitution between intermediate goods. The corresponding price index for the final consumption good is

$$
P_{t}=\left(\int_{0}^{1} P_{t}(i)^{1-\theta} d i\right)^{\frac{1}{1-\theta}},
$$

where $P_{t}(i)$ is the price of the intermediate good $i$. The optimal demand for $Y_{t}(i)$ is given by

$$
Y_{t}(i)=\left(\frac{P_{t}(i)}{P_{t}}\right)^{-\theta} Y_{t} .
$$

Monopolistically competitive firms produce intermediate goods using the production function, $Y_{t}(i)=$ $a_{t} N_{t}(i)$, where $N_{t}(i)$ denotes the labor hours employed by firm $i$ and $a_{t}$ represents exogenous economy-wide productivity. Prices are sticky. A firm adjusts its price, $P_{t}(i)$, with probability $1-\alpha$ each period, to maximize the present discounted value of future profits:

$$
E_{t} \sum_{k=0}^{\infty} \alpha^{k} Q_{t, t+k}\left[P_{t}(i)-\frac{W_{t+k}}{A_{t+k}}\right] Y_{t+k}(i) .
$$




\section{A.3 Government}

Each period, the government collects lump-sum tax revenues $\tau_{t}$ and issues one-period nominal bonds $B_{t}$ to finance its consumption $G_{t}$, and interest payments. Accordingly, the flow budget constraint is given by:

$$
\frac{B_{t}}{P_{t}}=R_{t-1} \frac{B_{t-1}}{P_{t}}+G_{t}-\tau_{t} .
$$

For simplicity, we assume $G_{t}=0$, which is inconsequential for our theoretical results. The flow budget constraint can be rewritten as:

$$
R_{t}^{-1} b_{t}=b_{t-1} \frac{1}{\pi_{t}}-\tau_{t},
$$

where $b_{t} \equiv R_{t} \frac{B_{t}}{P_{t}}$ denotes the real maturity value of government debt.

The monetary and fiscal policies are described by simple rules. The monetary authority responds to deviations of the inflation rate from its time-varying target rate, $\pi_{t}^{*}$, by setting the nominal interest rate according to:

$$
\frac{R_{t}}{\bar{R}}=\left(\frac{\pi_{t}}{\pi_{t}^{*}}\right)^{\phi}
$$

where $\bar{R}$ is the steady-state value of $R_{t}$. Similarly, the fiscal authority sets the tax revenues according to:

$$
\frac{\tau_{t}}{\bar{\tau}}=\left(\frac{b_{t-1}}{\bar{b}}\right)^{\psi}
$$

where $\bar{\tau}$ and $\bar{b}$ are respectively the steady state value of $\tau_{t}$ and $b_{t}$.

\section{A.4 Approximate Model}

We log-linearize the equilibrium conditions around the non-stochastic steady state with values $\{\bar{\pi}, \bar{Y}, \bar{R}, \bar{b}, \bar{\tau}\}$. In particular, we assume that inflation is zero in the steady state: $\bar{\pi}=0$. Since the log-linearized model is standard, we omit a detailed derivation. The approximate model is characterized by the following equations:

$$
\begin{aligned}
& \hat{Y}_{t}=E_{t} \hat{Y}_{t+1}-\left(\hat{R}_{t}-E_{t} \hat{\pi}_{t+1}\right)-E_{t}\left[\Delta \hat{d}_{t+1}\right], \\
& \hat{\pi}_{t}=\kappa\left(\hat{Y}_{t}-\hat{Y}_{t}^{n}\right)+\beta E_{t} \hat{\pi}_{t+1}, \\
& \hat{R}_{t}=\phi\left(\hat{\pi}_{t}-\hat{\pi}_{t}^{*}\right), \\
& \hat{\tau}_{t}=\psi \hat{b}_{t-1}, \\
& \hat{b}_{t}=\beta^{-1} \hat{b}_{t-1}-\beta^{-1} \bar{b}_{t}-\beta^{-1} \hat{\tau}_{t}+\bar{b} \hat{R}_{t} .
\end{aligned}
$$

In the equations above, we use $\hat{X}_{t}$ to denote the log deviation of a variable $X_{t}$ from its steady state $\bar{X}$ $\left(\hat{X}_{t}=\ln X_{t}-\ln \bar{X}\right)$, except for two fiscal variables, $\hat{b}_{t}$ and $\hat{\tau}_{t}$. Following Woodford (2003), we let them represent respectively the deviation of the maturity value of government debt and of government tax revenues (net of transfers) from their steady-state levels, measured as a percentage of steady-state output: $\hat{b}_{t}=\left(b_{t}-\bar{b}\right) / \bar{Y}$ and $\hat{\tau}_{t}=\left(\tau_{t}-\bar{\tau}\right) / \bar{Y}$. In our simple model, (the log-deviation of) the natural level of output and the slope of the Phillips curve are respectively given as $\hat{Y}_{t}^{n}=\hat{a}_{t}$ and $\kappa=(1-\alpha)(1-\alpha \beta) / \alpha$.

The model can be reduced to a dynamic system of $\left\{\hat{\pi}_{t}, \hat{b}_{t}, \tilde{Y}_{t}\right\}$ :

$$
\tilde{Y}_{t}=E_{t} \tilde{Y}_{t+1}-\phi\left(\hat{\pi}_{t}-\hat{\pi}_{t}^{*}\right)+E_{t} \hat{\pi}_{t+1}+\hat{r}_{t}^{*},
$$




$$
\begin{aligned}
& \hat{\pi}_{t}=\kappa \tilde{Y}_{t}+\beta E_{t} \hat{\pi}_{t+1}, \\
& \hat{b}_{t}=\beta^{-1}(1-\psi) \hat{b}_{t-1}-\bar{b}\left(\beta^{-1}-\phi\right) \hat{\pi}_{t}-\bar{b} \phi \hat{\pi}_{t}^{*},
\end{aligned}
$$

where $\tilde{Y}_{t} \equiv \hat{Y}_{t}-\hat{Y}_{t}^{n}$ represents the output gap and $\hat{r}_{t}^{*}$ is a linear combination of all non-policy shocks (of both supply and demand types). It is often referred to as the natural rate of interest because it is the real interest rate that would prevail under flexible prices. In our simple model, it is specifically given as:

$$
\hat{r}_{t}^{*}=E_{t}\left[\Delta \hat{a}_{t+1}\right]-E_{t}\left[\Delta \hat{d}_{t+1}\right]
$$

Note that demand-type shocks raise $\hat{r}_{t}^{*}$, while supply-type shocks lower $\hat{r}_{t}^{*}$.

\section{B Solution of the Simple Model}

In this section, we solve for the equilibrium time paths of $\left\{\hat{\pi}_{t}, \hat{b}_{t}, \tilde{Y}_{t}\right\}$ given exogenous variables summarized by the policy and non-policy shocks, $\left\{\hat{\pi}_{t}^{*}, \hat{r}_{t}^{*}\right\}$. To this end, we assume the exogenous random variables follow AR(1) processes:

$$
\begin{aligned}
\hat{\pi}_{t}^{*} & =\rho_{\pi} \hat{\pi}_{t-1}^{*}+\varepsilon_{\pi, t}, \\
\hat{r}_{t}^{*} & =\rho_{r} \hat{r}_{t-1}^{*}+\varepsilon_{r, t} .
\end{aligned}
$$

We first write (12) in state space form:

$$
\begin{aligned}
\left(\begin{array}{lll}
1 & 1 & 0 \\
0 & \beta & 0 \\
0 & 0 & 1
\end{array}\right) E_{t}\left(\begin{array}{c}
\tilde{Y}_{t+1} \\
\hat{\pi}_{t+1} \\
\hat{b}_{t}
\end{array}\right) & =\left(\begin{array}{ccc}
1 & \phi & 0 \\
-\kappa & 1 & 0 \\
0 & -\bar{b}\left(\beta^{-1}-\phi\right) & \beta^{-1}(1-\psi)
\end{array}\right)\left(\begin{array}{c}
\tilde{Y}_{t} \\
\hat{\pi}_{t} \\
\hat{b}_{t-1}
\end{array}\right) \\
& +\left(\begin{array}{cc}
-\phi & -1 \\
0 & 0 \\
-\bar{b} \phi & 0
\end{array}\right)\left(\begin{array}{c}
\hat{\pi}_{t}^{*} \\
r_{t}^{*}
\end{array}\right)
\end{aligned}
$$

We then pre-multiply $\left(\begin{array}{ccc}1 & 1 & 0 \\ 0 & \beta & 0 \\ 0 & 0 & 1\end{array}\right)^{-1}$ to both sides of the equation (13):

$$
E_{t}\left(\begin{array}{c}
\tilde{Y}_{t+1} \\
\hat{\pi}_{t+1} \\
\hat{b}_{t}
\end{array}\right)=\underbrace{\left(\begin{array}{ccc}
\kappa \beta^{-1}+1 & \phi-\beta^{-1} & 0 \\
-\kappa \beta^{-1} & \beta^{-1} & 0 \\
0 & -\bar{b}\left(\beta^{-1}-\phi\right) & \beta^{-1}(1-\psi)
\end{array}\right)}_{=G}\left(\begin{array}{c}
\tilde{Y}_{t} \\
\hat{\pi}_{t} \\
\hat{b}_{t-1}
\end{array}\right)+\left(\begin{array}{cc}
-\phi & -1 \\
0 & 0 \\
-\bar{b} \phi & 0
\end{array}\right)\left(\begin{array}{c}
\hat{\pi}_{t}^{*} \\
\hat{r}_{t}^{*}
\end{array}\right)
$$

The coefficient matrix, $G$, can be decomposed as $G=V D V^{-1}$, where $D$ is a diagonal matrix whose elements are the eigenvalues of $G$. The system then can be written as:

$$
E_{t}\left(\begin{array}{c}
\tilde{Y}_{t+1} \\
\hat{\pi}_{t+1} \\
\hat{b}_{t}
\end{array}\right)=V\left(\begin{array}{ccc}
e_{1} & 0 & 0 \\
0 & e_{2} & 0 \\
0 & 0 & e_{3}
\end{array}\right) V^{-1}\left(\begin{array}{c}
\tilde{Y}_{t} \\
\hat{\pi}_{t} \\
\hat{b}_{t-1}
\end{array}\right)+\left(\begin{array}{cc}
-\phi & -1 \\
0 & 0 \\
-\bar{b} \phi & 0
\end{array}\right)\left(\begin{array}{c}
\hat{\pi}_{t}^{*} \\
\hat{r}_{t}^{*}
\end{array}\right),
$$


where

$$
\begin{gathered}
e_{1}=\frac{1}{2 \beta}\left(\beta+\kappa+1+\sqrt{(\beta+\kappa+1)^{2}-4 \beta(1+\kappa \phi)}\right) \\
e_{2}=\beta^{-1}(1-\psi) \\
e_{3}=\frac{1}{2 \beta}\left(\beta+\kappa+1-\sqrt{(\beta+\kappa+1)^{2}-4 \beta(1+\kappa \phi)}\right) \\
V=\left(\begin{array}{ccc}
v_{11} & v_{12} & v_{13} \\
v_{21} & v_{22} & v_{23} \\
1 & 1 & 1
\end{array}\right) \quad \text { and } V^{-1}=\left(\begin{array}{lll}
q_{11} & q_{12} & 0 \\
q_{21} & q_{22} & 1 \\
q_{31} & q_{32} & 0
\end{array}\right) .
\end{gathered}
$$

The elements of $V$ and $V^{-1}$ are nonlinear functions of the model parameters. For later use, we note that:

$$
v_{23}=\frac{2(1-\psi)-\left(\beta+\kappa+1-\sqrt{(\beta+\kappa+1)^{2}-4 \beta\left(1+\kappa \phi_{\pi}\right)}\right)}{2 \bar{b}(1-\beta \phi)} .
$$

Finally, letting $X_{t} \equiv\left(\begin{array}{ccc}x_{1, t} & x_{2, t} & x_{3, t}\end{array}\right)^{T} \equiv V^{-1}\left(\begin{array}{ccc}\tilde{Y}_{t} & \hat{\pi}_{t} & \hat{b}_{t-1}\end{array}\right)^{T}$, we rewrite the system as:

$$
E_{t} X_{t+1}=\left(\begin{array}{ccc}
e_{1} & 0 & 0 \\
0 & e_{2} & 0 \\
0 & 0 & e_{3}
\end{array}\right) X_{t}+\left(\begin{array}{cc}
-\phi q_{11} & -q_{11} \\
-\phi\left(q_{21}+\bar{b}\right) & -q_{21} \\
-\phi q_{31} & -q_{31}
\end{array}\right)\left(\begin{array}{c}
\hat{\pi}_{t}^{*} \\
\hat{r}_{t}^{*}
\end{array}\right) .
$$

Each element of $X_{t}$ is given by:

$$
\begin{aligned}
& x_{1, t}=q_{11} \tilde{Y}_{t}+q_{12} \hat{\pi}_{t}, \\
& x_{2, t}=q_{21} \tilde{Y}_{t}+q_{22} \hat{\pi}_{t}+\hat{b}_{t-1}, \\
& x_{3, t}=q_{31} \tilde{Y}_{t}+q_{32} \hat{\pi}_{t} .
\end{aligned}
$$

\section{B.1 Active Monetary and Passive Fiscal Policy}

Under AMPF, $e_{1}$ and $e_{3}$ are outside the unit circle, while $e_{2}$ is inside the circle. We thus use the first and third rows of the system (14) to draw linear restrictions between model variables. Substituting out the future values of $x_{1, t}$ and $x_{3, t}$ recursively, we obtain:

$$
\begin{aligned}
& x_{1, t}=\frac{1}{e_{1}} \sum_{k=0}^{\infty}\left(\frac{1}{e_{1}}\right)^{k} E_{t} z_{1, t+k}^{*}, \\
& x_{3, t}=\frac{1}{e_{3}} \sum_{k=0}^{\infty}\left(\frac{1}{e_{3}}\right)^{k} E_{t} z_{3, t+k}^{*},
\end{aligned}
$$

where

$$
\begin{aligned}
& z_{1, t}^{*}=\phi q_{11} \hat{\pi}_{t}^{*}+q_{11} \hat{r}_{t}^{*}, \\
& z_{3, t}^{*}=\phi q_{31} \hat{\pi}_{t}^{*}+q_{31} \hat{r}_{t}^{*} .
\end{aligned}
$$


These equations imply:

$$
\begin{aligned}
& E_{t} z_{1, t+k}^{*}=\phi q_{11} \rho_{\pi}^{k} \hat{\pi}_{t}^{*}+q_{11} \rho_{r}^{k} \hat{r}_{t}^{*} \\
& E_{t} z_{3, t+k}^{*}=\phi q_{31} \rho_{\pi}^{k} \hat{\pi}_{t}^{*}+q_{31} \rho_{r}^{k} \hat{r}_{t}^{*}
\end{aligned}
$$

Plugging these equations into (15) and (16), we obtain:

$$
\begin{aligned}
& x_{1, t}=\frac{1}{e_{1}} \sum_{k=0}^{\infty}\left(\frac{1}{e_{1}}\right)^{k} E_{t} z_{1, t+k}^{*}=\phi q_{11} \frac{1}{e_{1}-\rho_{\pi}} \hat{\pi}_{t}^{*}+q_{11} \frac{1}{e_{1}-\rho_{r}} \hat{r}_{t}^{*}, \\
& x_{3, t}=\frac{1}{e_{3}} \sum_{k=0}^{\infty}\left(\frac{1}{e_{3}}\right)^{k} E_{t} z_{3, t+k}^{*}=\phi q_{31} \frac{1}{e_{3}-\rho_{\pi}} \hat{\pi}_{t}^{*}+q_{31} \frac{1}{e_{3}-\rho_{r}} \hat{r}_{t}^{*},
\end{aligned}
$$

which leads to:

$$
\begin{aligned}
& \tilde{Y}_{t}=-\frac{q_{12}}{q_{11}} \hat{\pi}_{t}+\phi \frac{1}{e_{1}-\rho_{\pi}} \hat{\pi}_{t}^{*}+\frac{1}{e_{1}-\rho_{r}} \hat{r}_{t}^{*} \\
& \tilde{Y}_{t}=-\frac{q_{32}}{q_{31}} \hat{\pi}_{t}+\phi \frac{1}{e_{3}-\rho_{\pi}} \hat{\pi}_{t}^{*}+\frac{1}{e_{3}-\rho_{r}} \hat{r}_{t}^{*}
\end{aligned}
$$

Since the above system has two equations and two endogenous variables, we can easily solve for $\hat{\pi}_{t}$ and $\tilde{Y}_{t}$. Moreover, $\hat{\pi}_{t}$ and $\tilde{Y}_{t}$ do not depend on $\hat{b}_{t-1}$. We use the method of undetermined coefficients and obtain:

$$
\begin{aligned}
& \hat{\pi}_{t}=\Phi(\phi) \hat{\pi}_{t}^{*}+\Gamma(\phi) \hat{r}_{t}^{*} \\
& \tilde{Y}_{t}=\Phi^{Y}(\phi) \hat{\pi}_{t}^{*}+\Gamma^{Y}(\phi) \hat{r}_{t}^{*}
\end{aligned}
$$

where

$$
\begin{aligned}
\Phi(\phi) & \equiv \frac{\kappa \phi}{\kappa\left(\phi-\rho_{\pi}\right)+\left(1-\rho_{\pi}\right)\left(1-\beta \rho_{\pi}\right)} \\
\Gamma(\phi) & \equiv \frac{\kappa}{\kappa\left(\phi-\rho_{r}\right)+\left(1-\rho_{r}\right)\left(1-\beta \rho_{r}\right)} \\
\Phi^{Y}(\phi) & \equiv \frac{\phi\left(1-\beta \rho_{\pi}\right)}{\kappa\left(\phi-\rho_{\pi}\right)+\left(1-\rho_{\pi}\right)\left(1-\beta \rho_{\pi}\right)} \\
\Gamma^{Y}(\phi) & \equiv \frac{\left(1-\beta \rho_{r}\right)}{\kappa\left(\phi-\rho_{r}\right)+\left(1-\rho_{r}\right)\left(1-\beta \rho_{r}\right)} .
\end{aligned}
$$

\section{B.2 Passive Monetary and Active Fiscal Policy}

We consider the case in which $\phi_{\pi} \in[0,1)$ and $\psi \in(-\infty, \bar{\psi})$ where $\bar{\psi} \equiv 1-\beta$ is the upper bound for active fiscal policy. We then can show that $e_{1}>1, e_{2}>1$ and $e_{3} \in(0,1)$ in that parameter space. Consequently the first two rows in (14) provide linear restrictions. From the rows, we obtain:

$$
\begin{aligned}
& x_{1, t}=\frac{1}{e_{1}} \sum_{k=0}^{\infty}\left(\frac{1}{e_{1}}\right)^{k} E_{t} z_{1, t+k}^{*}, \\
& x_{2, t}=\frac{1}{e_{2}} \sum_{k=0}^{\infty}\left(\frac{1}{e_{2}}\right)^{k} E_{t} z_{2, t+k}^{*},
\end{aligned}
$$


where

$$
\begin{aligned}
& z_{1, t}^{*}=\phi q_{11} \hat{\pi}_{t}^{*}+q_{11} \hat{r}_{t}^{*}, \\
& z_{2, t}^{*}=\phi\left(q_{21}+\bar{b}\right) \hat{\pi}_{t}^{*}+q_{21} \hat{r}_{t}^{*} .
\end{aligned}
$$

The equations above imply:

$$
\begin{aligned}
& E_{t} z_{1, t+k}^{*}=\phi q_{11} \rho_{\pi}^{k} \hat{\pi}_{t}^{*}+q_{11} \rho_{r}^{k} \hat{r}_{t}^{*} \\
& E_{t} z_{2, t+k}^{*}=\phi\left(q_{21}+\bar{b}\right) \rho_{\pi}^{k} \hat{\pi}_{t}^{*}+q_{21} \rho_{r}^{k} \hat{r}_{t}^{*}
\end{aligned}
$$

Plugging these equations into (15) and (16), we obtain:

$$
\begin{aligned}
& x_{1, t}=\frac{1}{e_{1}} \sum_{k=0}^{\infty}\left(\frac{1}{e_{1}}\right)^{k} E_{t} z_{1, t+k}^{*}=\phi q_{11} \frac{1}{e_{1}-\rho_{\pi}} \hat{\pi}_{t}^{*}+q_{11} \frac{1}{e_{1}-\rho_{r}} \hat{r}_{t}^{*} \\
& x_{2, t}=\frac{1}{e_{2}} \sum_{k=0}^{\infty}\left(\frac{1}{e_{2}}\right)^{k} E_{t} z_{2, t+k}^{*}=\phi\left(q_{21}+\bar{b}\right) \frac{1}{e_{2}-\rho_{\pi}} \hat{\pi}_{t}^{*}+q_{21} \frac{1}{e_{2}-\rho_{r}} \hat{r}_{t}^{*}
\end{aligned}
$$

Equation (19) implies:

$$
\tilde{Y}_{t}=-\frac{q_{12}}{q_{11}} \hat{\pi}_{t}+\phi \frac{1}{e_{1}-\rho_{\pi}} \hat{\pi}_{t}^{*}+\frac{1}{e_{1}-\rho_{r}} \hat{r}_{t}^{*}
$$

We plug (21) into (20) to get:

$$
q_{21}\left[-\frac{q_{12}}{q_{11}} \hat{\pi}_{t}+\phi \frac{1}{e_{1}-\rho_{\pi}} \hat{\pi}_{t}^{*}+\frac{1}{e_{1}-\rho_{r}} r_{t}^{*}\right]+q_{22} \hat{\pi}_{t}+\hat{b}_{t-1}=\phi\left(q_{21}+\bar{b}\right) \frac{1}{e_{2}-\rho_{\pi}} \hat{\pi}_{t}^{*}+q_{21} \frac{1}{e_{2}-\rho_{r}} \hat{r}_{t}^{*}
$$

Solving for $\hat{\pi}_{t}$, we obtain $\hat{\pi}_{t}$ as a function of state variables, $\left\{\hat{b}_{t-1}, \hat{\pi}_{t}^{*}, r_{t}^{*}\right\}$ :

$$
\hat{\pi}_{t}=\Omega \hat{b}_{t-1}-\Omega \phi\left[\left(q_{21}+\bar{b}\right) \frac{1}{e_{2}-\rho_{\pi}}-q_{21} \frac{1}{e_{1}-\rho_{\pi}}\right] \hat{\pi}_{t}^{*}+\Omega q_{21}\left[\frac{1}{e_{1}-\rho_{r}}-\frac{1}{e_{2}-\rho_{r}}\right] r_{t}^{*}
$$

where

$$
\begin{aligned}
\Omega & =\frac{q_{11}}{q_{12} q_{21}-q_{11} q_{22}} \\
q_{21} & =\frac{\bar{b} \kappa(1-\beta \phi)}{\psi^{2}+(\beta+\kappa-1) \psi-\kappa(1-\beta \phi)} .
\end{aligned}
$$

For further analysis, it is useful to express the coefficients on the state variables in terms of model parameters. To this end, we use the results in the following lemmas.

Lemma 1: $\Omega=\frac{\beta\left(e_{2}-e_{3}\right)}{b(1-\beta \phi)}>0$.

Proof of Lemma 1: Note that

$$
\begin{aligned}
& \frac{q_{13} q_{21}-q_{11} q_{23}}{\operatorname{det}\left(V^{-1}\right)}=v_{23}, \\
& \frac{q_{11} q_{22}-q_{12} q_{21}}{\operatorname{det}\left(V^{-1}\right)}=v_{33} .
\end{aligned}
$$


Therefore,

$$
\begin{aligned}
0 \times q_{21}-q_{11} & =-q_{11}=\operatorname{det}\left(V^{-1}\right) \times v_{23}, \\
q_{11} q_{22}-q_{12} q_{21} & =\operatorname{det}\left(V^{-1}\right) \times v_{33}=\operatorname{det}\left(V^{-1}\right) \times 1 .
\end{aligned}
$$

It follows that

$$
\begin{aligned}
\Omega & =\frac{q_{11}}{q_{12} q_{21}-q_{11} q_{22}}=v_{23} \\
& =\frac{2(1-\psi)-\left(\beta+\kappa+1-\sqrt{(\beta+\kappa+1)^{2}-4 \beta(1+\kappa \phi)}\right)}{2 \bar{b}(1-\beta \phi)} \\
& =\frac{2(1-\psi)-2 \beta e_{3}}{2 \bar{b}(1-\beta \phi)}=\frac{\beta\left(e_{2}-e_{3}\right)}{\bar{b}(1-\beta \phi)}>0 .
\end{aligned}
$$

Lemma 2: $\Omega q_{21}\left(e_{1}-e_{2}\right)=-\kappa \beta^{-1}$.

Proof of Lemma 2: We have

$$
\begin{gathered}
\quad \Omega q_{21}\left(e_{1}-e_{2}\right)=\frac{\beta\left(e_{2}-e_{3}\right)}{\bar{b}(1-\beta \phi)} \frac{\bar{b} \kappa(1-\beta \phi)}{\psi^{2}+(\beta+\kappa-1) \psi-\kappa(1-\beta \phi)}\left(e_{1}-e_{2}\right) \\
=\kappa \beta \frac{\left(e_{2}-e_{3}\right)\left(e_{1}-e_{2}\right)}{\psi^{2}+(\beta+\kappa-1) \psi-\kappa(1-\beta \phi)}=\kappa \beta \frac{e_{1} e_{2}-e_{2}^{2}-e_{1} e_{3}+e_{2} e_{3}}{\psi^{2}+(\beta+\kappa-1) \psi-\kappa(1-\beta \phi)} \\
=\kappa \beta \frac{e_{2}\left(e_{1}+e_{3}-e_{2}\right)-e_{1} e_{3}}{\psi^{2}+(\beta+\kappa-1) \psi-\kappa(1-\beta \phi)}=\kappa \beta \frac{\frac{(1-\psi)(\beta+\kappa+\psi)}{\beta^{2}}-e_{1} e_{3}}{\psi^{2}+(\beta+\kappa-1) \psi-\kappa(1-\beta \phi)} \\
=\kappa \beta \frac{\frac{(1-\psi)(\beta+\kappa+\psi)}{\beta^{2}}-\frac{1+\kappa \phi}{\beta}}{\psi^{2}+(\beta+\kappa-1) \psi-\kappa(1-\beta \phi)}=\kappa \beta \frac{\frac{1}{\beta^{2}}[(1-\psi)(\beta+\kappa+\psi)-\beta-\kappa \beta \phi]}{\psi^{2}+(\beta+\kappa-1) \psi-\kappa(1-\beta \phi)} \\
=\kappa \beta^{-1} \frac{\left[\psi^{2}+(\beta+\kappa-1) \psi-\kappa(1-\beta \phi)\right]}{\psi^{2}+(\beta+\kappa-1) \psi-\kappa(1-\beta \phi)}=-\kappa \beta^{-1} .
\end{gathered}
$$

Using the results from these two lemmas, we can simplify (22) as:

$$
\hat{\pi}_{t}=\Omega(\phi, \psi) \hat{b}_{t-1}-\Phi(\phi, \psi) \hat{\pi}_{t}^{*}+\Gamma(\phi, \psi) \hat{r}_{t}^{*}
$$

where

$$
\begin{aligned}
\Omega(\phi, \psi) & \equiv \frac{\beta\left(e_{2}-e_{3}\right)}{\bar{b}(1-\beta \phi)}, \\
\Phi(\phi, \psi) & \equiv \phi \times \Theta(\phi, \psi), \quad \text { where } \Theta(\phi, \psi) \equiv \frac{\Omega \bar{b}\left(e_{1}-\rho_{\pi}\right)-\kappa \beta^{-1}}{\left(e_{1}-\rho_{\pi}\right)\left(e_{2}-\rho_{\pi}\right)}, \\
\Gamma(\phi, \psi) & \equiv \frac{\kappa \beta^{-1}}{\left(e_{1}-\rho_{r}\right)\left(e_{2}-\rho_{r}\right)} .
\end{aligned}
$$

It then follows that the law of motion for $\hat{b}_{t}$ is given as:

$$
\hat{b}_{t}=e_{3} \hat{b}_{t-1}-\bar{b} \phi\left[1-\left(\beta^{-1}-\phi\right) \Theta\right] \hat{\pi}_{t}^{*}-\bar{b}\left(\beta^{-1}-\phi\right) \Gamma \hat{r}_{t}^{*}
$$




\section{B.3 Passive Monetary and Passive Fiscal Policy}

Finally, we consider the case in which $\phi_{\pi} \in[0,1)$ and $\psi \in(\bar{\psi}, \infty)$. Then, only one root $\left(e_{1}\right)$ is explosive and there will exist multiple solutions to the model, which makes it more difficult to obtain analytical results. For analytical tractability, we focus on a case in which the shocks are i.i.d.

Our solution methodology follows that of Lubik and Schorfheide (2003). Let us first introduce two new variables, $\xi_{t}^{Y} \equiv E_{t} \tilde{Y}_{t+1}$ and $\xi_{t}^{\pi} \equiv E_{t} \hat{\pi}_{t+1}$, and rewrite the model as:

$$
\begin{aligned}
\left(\begin{array}{lllll}
1 & 0 & 0 & 0 & 0 \\
0 & 1 & 0 & 0 & 0 \\
0 & 0 & 1 & 1 & 0 \\
0 & 0 & 0 & \beta & 0 \\
0 & 0 & 0 & 0 & 1
\end{array}\right)\left(\begin{array}{c}
\tilde{Y}_{t} \\
\hat{\pi}_{t} \\
\xi_{t}^{Y} \\
\xi_{t}^{\pi} \\
\hat{b}_{t}
\end{array}\right)= & \left(\begin{array}{ccccc}
0 & 0 & 1 & 0 & 0 \\
0 & 0 & 0 & 1 & 0 \\
0 & 0 & 1 & \phi & 0 \\
0 & 0 & -\kappa & 1 & 0 \\
0 & 0 & 0 & -\bar{b}\left(\beta^{-1}-\phi\right) \\
\beta^{-1}(1-\psi)
\end{array}\right)\left(\begin{array}{c}
\tilde{Y}_{t-1} \\
\hat{\pi}_{t-1} \\
\xi_{t-1}^{Y} \\
\xi_{t-1}^{\pi} \\
\hat{b}_{t-1}
\end{array}\right) \\
& +\left(\begin{array}{cc}
0 & 0 \\
0 & 0 \\
-\phi & -1 \\
0 & 0 \\
-\bar{b} \phi & 0
\end{array}\right)\left(\begin{array}{c}
\hat{\pi}_{t}^{*} \\
\hat{r}_{t}^{*}
\end{array}\right)+\left(\begin{array}{cc}
0 \\
1 \\
-\kappa & 1 \\
0 & -\bar{b}\left(\beta^{-1}-\phi\right)
\end{array}\right)\left(\begin{array}{l}
\eta_{t}^{Y} \\
\eta_{t}^{\pi}
\end{array}\right)
\end{aligned}
$$

Since the system is block-recursive, we solve the lower block first:

$$
\begin{aligned}
\left(\begin{array}{lll}
1 & 1 & 0 \\
0 & \beta & 0 \\
0 & 0 & 1
\end{array}\right)\left(\begin{array}{l}
\xi_{t}^{Y} \\
\xi_{t}^{\pi} \\
\hat{b}_{t}
\end{array}\right)= & \left(\begin{array}{ccc}
1 & \phi & 0 \\
-\kappa & 1 & 0 \\
0 & -\bar{b}\left(\beta^{-1}-\phi\right) & \beta^{-1} \\
(1-\psi)
\end{array}\right)\left(\begin{array}{c}
\xi_{t-1}^{Y} \\
\xi_{t-1}^{\pi} \\
\hat{b}_{t-1}
\end{array}\right) \\
& +\left(\begin{array}{cc}
-\phi & -1 \\
0 & 0 \\
-\bar{b} \phi & 0
\end{array}\right)\left(\begin{array}{c}
\hat{\pi}_{t}^{*} \\
\hat{r}_{t}^{*}
\end{array}\right)+\left(\begin{array}{cc}
1 & \phi \\
-\kappa & 1 \\
0 & -\bar{b}\left(\beta^{-1}-\phi\right)
\end{array}\right)\left(\begin{array}{c}
\eta_{t}^{Y} \\
\eta_{t}^{\pi}
\end{array}\right)
\end{aligned}
$$

Pre-multiply $\left(\begin{array}{ccc}1 & 1 & 0 \\ 0 & \beta & 0 \\ 0 & 0 & 1\end{array}\right)^{-1}$ to both sides of the equation and obtain:

$$
\begin{aligned}
\left(\begin{array}{c}
\xi_{t}^{Y} \\
\xi_{t}^{\pi} \\
\hat{b}_{t}
\end{array}\right) & =\underbrace{\left(\begin{array}{ccc}
\kappa \beta^{-1}+1 & \phi-\beta^{-1} & 0 \\
-\kappa \beta^{-1} & \beta^{-1} & 0 \\
0 & -\bar{b}\left(\beta^{-1}-\phi\right) & \beta^{-1}(1-\psi)
\end{array}\right)}_{=G}\left(\begin{array}{c}
\xi_{t-1}^{Y} \\
\xi_{t-1}^{\pi} \\
\hat{b}_{t-1}
\end{array}\right) \\
& +\left(\begin{array}{cc}
-\phi & -1 \\
0 & 0 \\
-\bar{b} \phi & 0
\end{array}\right)\left(\begin{array}{c}
\hat{\pi}_{t}^{*} \\
\hat{r}_{t}^{*}
\end{array}\right)+\left(\begin{array}{cc}
\kappa \beta^{-1}+1 & \phi-\beta^{-1} \\
-\kappa \beta^{-1} & \beta^{-1} \\
0 & -\bar{b}\left(\beta^{-1}-\phi\right)
\end{array}\right)\left(\begin{array}{c}
\eta_{t}^{Y} \\
\eta_{t}^{\pi}
\end{array}\right) .
\end{aligned}
$$

As before, the coefficient matrix, $G$, can be decomposed as $G=V D V^{-1}$. The system then can be written as:

$$
\left(\begin{array}{l}
\xi_{t}^{Y} \\
\xi_{t}^{\pi} \\
\hat{b}_{t}
\end{array}\right)=V\left(\begin{array}{ccc}
e_{1} & 0 & 0 \\
0 & e_{2} & 0 \\
0 & 0 & e_{3}
\end{array}\right) V^{-1}\left(\begin{array}{c}
\xi_{t-1}^{Y} \\
\xi_{t-1}^{\pi} \\
\hat{b}_{t-1}
\end{array}\right)
$$




$$
+\left(\begin{array}{cc}
-\phi & -1 \\
0 & 0 \\
-\bar{b} \phi & 0
\end{array}\right)\left(\begin{array}{c}
\hat{\pi}_{t}^{*} \\
\hat{r}_{t}^{*}
\end{array}\right)+\left(\begin{array}{cc}
\kappa \beta^{-1}+1 & \phi-\beta^{-1} \\
-\kappa \beta^{-1} & \beta^{-1} \\
0 & -\bar{b}\left(\beta^{-1}-\phi\right)
\end{array}\right)\left(\begin{array}{c}
\eta_{t}^{Y} \\
\eta_{t}^{\pi}
\end{array}\right)
$$

Note that $G$ is the same as under AMPF and PMAF, and hence $e_{1}, e_{2}, e_{3}, V$ and $V^{-1}$ are the same as under AMPF and PMAF. We can show that $e_{1}>1$, and $e_{2}, e_{3} \in(0,1)$. Let $X_{t}=\left(\begin{array}{c}x_{1, t} \\ x_{2, t} \\ x_{3, t}\end{array}\right)=V^{-1}\left(\begin{array}{c}\xi_{t}^{Y} \\ \xi_{t}^{\pi} \\ \hat{b}_{t}\end{array}\right)$, and rewrite the system as:

$$
\begin{aligned}
X_{t}= & \left(\begin{array}{ccc}
e_{1} & 0 & 0 \\
0 & e_{2} & 0 \\
0 & 0 & e_{3}
\end{array}\right) X_{t-1}+\left(\begin{array}{lll}
q_{11} & q_{12} & 0 \\
q_{21} & q_{22} & 1 \\
q_{31} & q_{32} & 0
\end{array}\right)\left(\begin{array}{cc}
-\phi & -1 \\
0 & 0 \\
-\bar{b} \phi & 0
\end{array}\right)\left(\begin{array}{c}
\hat{\pi}_{t}^{*} \\
\hat{r}_{t}^{*}
\end{array}\right) \\
& +\left(\begin{array}{lll}
q_{11} & q_{12} & 0 \\
q_{21} & q_{22} & 1 \\
q_{31} & q_{32} & 0
\end{array}\right)\left(\begin{array}{cc}
\kappa \beta^{-1}+1 & \phi-\beta^{-1} \\
-\kappa \beta^{-1} & \beta^{-1} \\
0 & -\bar{b}\left(\beta^{-1}-\phi\right)
\end{array}\right)\left(\begin{array}{c}
\eta_{t}^{Y} \\
\eta_{t}^{\pi}
\end{array}\right) .
\end{aligned}
$$

Note that $x_{1, t}$ in particular has an unstable root $e_{1}$ and satisfies:

$$
\begin{aligned}
x_{1, t}= & e_{1} x_{1, t-1}+\left(\begin{array}{ll}
-\phi q_{11} & -q_{11}
\end{array}\right)\left(\begin{array}{c}
\hat{\pi}_{t}^{*} \\
\hat{r}_{t}^{*}
\end{array}\right) \\
& +\left(\begin{array}{ll}
\kappa \beta^{-1}\left(q_{11}-q_{12}\right)+q_{11} & -\beta^{-1}\left(q_{11}-q_{12}\right)+\phi q_{11}
\end{array}\right)\left(\begin{array}{c}
\eta_{t}^{Y} \\
\eta_{t}^{\pi}
\end{array}\right) .
\end{aligned}
$$

Using the singular value decomposition, one can obtain:

$$
\begin{aligned}
& \left(\kappa \beta^{-1}\left(q_{11}-q_{12}\right)+q_{11}-\beta^{-1}\left(q_{11}-q_{12}\right)+\phi q_{11}\right) \\
= & \left(\begin{array}{ll}
d & 0
\end{array}\right)\left(\begin{array}{cc}
\frac{\kappa \beta^{-1}\left(q_{11}-q_{12}\right)+q_{11}}{d} & \frac{-\beta^{-1}\left(q_{11}-q_{12}\right)+\phi q_{11}}{d} \\
v_{0}\left(\frac{-\beta^{-1}\left(q_{11}-q_{12}\right)+\phi q_{11}}{d}\right) & -v_{0}\left(\frac{\kappa \beta^{-1}\left(q_{11}-q_{12}\right)+q_{11}}{d}\right)
\end{array}\right),
\end{aligned}
$$

where a singular value $d$ is given as:

$$
d=\sqrt{\left[\kappa \beta^{-1}\left(q_{11}-q_{12}\right)+q_{11}\right]^{2}+\left[-\beta^{-1}\left(q_{11}-q_{12}\right)+\phi q_{11}\right]^{2}},
$$

and $v_{0}=1$ or -1 . Since one of the singular values is zero, $v_{0}$ is not uniquely determined. Now apply Proposition 1 of Lubik and Schorfheide (2003; 2004). We then obtain:

$$
\begin{aligned}
\left(\begin{array}{c}
\eta_{t}^{Y} \\
\eta_{t}^{\pi}
\end{array}\right)= & -\left(\begin{array}{c}
\frac{\kappa \beta^{-1}\left(q_{11}-q_{12}\right)+q_{11}}{d} \\
\frac{-\beta^{-1}\left(q_{11}-q_{12}\right)+\phi q_{11}}{d}
\end{array}\right) \times \frac{1}{d} \times 1 \times\left(\begin{array}{ll}
-\phi q_{11} & -q_{11}
\end{array}\right)\left(\begin{array}{c}
\hat{\pi}_{t}^{*} \\
\hat{r}_{t}^{*}
\end{array}\right) \\
& +\left(\begin{array}{c}
\frac{-\beta^{-1}\left(q_{11}-q_{12}\right)+\phi q_{11}}{d} \\
-\frac{\kappa \beta^{-1}\left(q_{11}-q_{12}\right)+q_{11}}{d}
\end{array}\right)\left\{M\left(\begin{array}{c}
\hat{\pi}_{t}^{*} \\
\hat{r}_{t}^{*}
\end{array}\right)+\zeta_{t}^{*}\right\} \\
= & -\frac{1}{d}\left(\begin{array}{c}
\frac{\kappa \beta^{-1}\left(q_{11}-q_{12}\right)+q_{11}}{d} \\
\frac{-\beta^{-1}\left(q_{11}-q_{12}\right)+\phi q_{11}}{d}
\end{array}\right)\left(\begin{array}{ll}
-\phi q_{11} & -q_{11}
\end{array}\right)\left(\begin{array}{c}
\hat{\pi}_{t}^{*} \\
\hat{r}_{t}^{*}
\end{array}\right)
\end{aligned}
$$




$$
+\left(\begin{array}{c}
\frac{-\beta^{-1}\left(q_{11}-q_{12}\right)+\phi q_{11}}{d} \\
-\frac{\kappa \beta^{-1}\left(q_{11}-q_{12}\right)+q_{11}}{d}
\end{array}\right)\left\{M\left(\begin{array}{c}
\hat{\pi}_{t}^{*} \\
\hat{r}_{t}^{*}
\end{array}\right)+\zeta_{t}^{*}\right\},
$$

where the elements of the matrix, $M=\left(\begin{array}{ll}M_{\pi} & M_{r}\end{array}\right)$, are new parameters introduced due to indeterminacy. Note that we chose $v_{0}=1$, which does not matter practically. This is because $v_{0}$ is multiplied to $M$ and $\zeta_{t}^{*}$ that are not pinned down by the structural parameters of the model. For $v_{0}=-1$, we can simply change the sign of $M$ and $\zeta_{t}^{*}$.

Moreover, the dynamics of inflation can be characterized as:

$$
\begin{aligned}
\hat{\pi}_{t}= & \xi_{t-1}^{\pi}+\eta_{t}^{\pi}=E_{t-1} \hat{\pi}_{t}+\eta_{t}^{\pi} \\
= & -\kappa \beta^{-1} \tilde{Y}_{t-1}+\beta^{-1} \hat{\pi}_{t-1}-\Phi_{\pi}(\phi, \psi) \hat{\pi}_{t}^{*}-\Gamma_{\pi}(\phi, \psi) \hat{r}_{t}^{*} \\
& +\Lambda_{\pi}(\phi, \psi)\left(M_{\pi} \hat{\pi}_{t}^{*}+M_{r} \hat{r}_{t}^{*}+\zeta_{t}^{*}\right),
\end{aligned}
$$

where

$$
\begin{aligned}
\Phi_{\pi}(\phi, \psi) & \equiv \phi \frac{q_{11}}{d^{2}}\left[\beta^{-1}\left(q_{11}-q_{12}\right)-\phi q_{11}\right] \\
\Gamma_{\pi}(\phi, \psi) & \equiv \frac{q_{11}}{d^{2}}\left[\beta^{-1}\left(q_{11}-q_{12}\right)-\phi q_{11}\right] \\
\Lambda_{\pi}(\phi, \psi) & =-\frac{\kappa \beta^{-1}\left(q_{11}-q_{12}\right)+q_{11}}{d} .
\end{aligned}
$$

Note that we substituted the Phillips curve (12) for $E_{t-1} \hat{\pi}_{t}$. Since the solution imposes a restriction on $\tilde{Y}_{t-1}$ and $\hat{\pi}_{t-1}$ such that $x_{1, t-1}=q_{11} \tilde{Y}_{t-1}+q_{12} \hat{\pi}_{t-1}=0$ for all $t$, the solution for inflation (25) is valid only when the state variables $\tilde{Y}_{t-1}$ and $\hat{\pi}_{t-1}$ satisfy $q_{11} \tilde{Y}_{t-1}+q_{12} \hat{\pi}_{t-1}=0$.

\section{B.4 Proofs}

\section{B.4.1 Proofs under AMPF}

Proof of Proposition 1 Showing $\Phi(\phi) \geq 0$ and $\Gamma(\phi) \geq 0$ is straightforward since $\kappa>0 ; \phi>\rho_{\pi}$; $\left(1-\rho_{\pi}\right)>0 ;\left(1-\beta \rho_{\pi}\right)>0 ; \phi>\rho_{r} ;\left(1-\rho_{r}\right)>0$; and $\left(1-\beta \rho_{r}\right)>0 .{ }^{32}$ In addition, it is also straightforward to show that $\Phi(\phi)$ is increasing in $\kappa$ by taking a partial derivative and:

$$
\Phi(\phi)>1, \quad \text { for } \kappa>\frac{\left(1-\rho_{\pi}\right)\left(1-\beta \rho_{\pi}\right)}{\rho_{\pi}} .
$$

Proof of Proposition 2 Take the partial derivative:

$$
\frac{\partial \Phi(\phi)}{\partial \phi}=\frac{-\kappa\left[\kappa \rho_{\pi}-\left(1-\rho_{\pi}\right)\left(1-\beta \rho_{\pi}\right)\right]}{\left[\kappa\left(\phi-\rho_{\pi}\right)+\left(1-\rho_{\pi}\right)\left(1-\beta \rho_{\pi}\right)\right]^{2}} .
$$

The denominator is always positive. Therefore, the sign of the numerator will determine the sign of the derivative. We thus have:

$$
\frac{\partial \Phi(\phi)}{\partial \phi}>0, \quad \text { for } 0<\kappa<\frac{\left(1-\rho_{\pi}\right)\left(1-\beta \rho_{\pi}\right)}{\rho_{\pi}}
$$

\footnotetext{
${ }^{32}$ See the previous section for the analytical solution for $\Phi(\phi), \Gamma(\phi)$, and other coefficients.
} 


$$
\begin{aligned}
& \frac{\partial \Phi(\phi)}{\partial \phi}=0, \quad \text { for } \kappa=\frac{\left(1-\rho_{\pi}\right)\left(1-\beta \rho_{\pi}\right)}{\rho_{\pi}} \\
& \frac{\partial \Phi(\phi)}{\partial \phi}<0, \quad \text { for } \kappa>\frac{\left(1-\rho_{\pi}\right)\left(1-\beta \rho_{\pi}\right)}{\rho_{\pi}} .
\end{aligned}
$$

It is trivial to show $\frac{\partial \Gamma(\phi)}{\partial \phi}<0$.

\section{B.4.2 Proofs under PMAF}

\section{Proof of Proposition 3}

(I) To prove $\Phi(\phi, \psi) \geq 0$, it suffices to show $\Theta(\phi, \psi)$ is positive since $0 \leq \phi<1$. Let us rewrite $\Theta(\phi, \psi)$ by substituting out $\Omega$ :

$$
\begin{gathered}
\Theta=\frac{\beta\left(e_{2}-e_{3}\right)\left(e_{1}-\rho_{\pi}\right)-\kappa \beta^{-1}(1-\beta \phi)}{\left(e_{1}-\rho_{\pi}\right)\left(e_{2}-\rho_{\pi}\right)(1-\beta \phi)} \\
=\frac{\beta\left(e_{2}-e_{3}\right)\left(e_{1}-e_{2}+e_{2}-\rho_{\pi}\right)-\kappa \beta^{-1}(1-\beta \phi)}{\left(e_{1}-\rho_{\pi}\right)\left(e_{2}-\rho_{\pi}\right)(1-\beta \phi)} \\
=\frac{\beta\left(e_{2}-e_{3}\right)\left(e_{1}-e_{2}\right)-\kappa \beta^{-1}(1-\beta \phi)+\beta\left(e_{2}-e_{3}\right)\left(e_{2}-\rho_{\pi}\right)}{\left(e_{1}-\rho_{\pi}\right)\left(e_{2}-\rho_{\pi}\right)(1-\beta \phi)} \\
=\frac{-\frac{1}{\beta}\left[\psi^{2}+(\beta+\kappa-1) \psi-\kappa(1-\beta \phi)\right]-\frac{\kappa}{\beta}(1-\beta \phi)+\beta\left(e_{2}-e_{3}\right)\left(e_{2}-\rho_{\pi}\right)}{\left(e_{1}-\rho_{\pi}\right)\left(e_{2}-\rho_{\pi}\right)(1-\beta \phi)} \\
=\frac{-\frac{1}{\beta}\left[\psi^{2}+(\beta+\kappa-1) \psi\right]+\beta\left(e_{2}-e_{3}\right)\left(e_{2}-\rho_{\pi}\right)}{\left(e_{1}-\rho_{\pi}\right)\left(e_{2}-\rho_{\pi}\right)(1-\beta \phi)}
\end{gathered}
$$

Substitute $\psi$ out using $\psi=1-\beta e_{2}$ :

$$
\begin{gathered}
\Theta=\frac{-\frac{1}{\beta}\left[\left(1-\beta e_{2}\right)^{2}+(\beta+\kappa-1)\left(1-\beta e_{2}\right)\right]+\beta\left(e_{2}-e_{3}\right)\left(e_{2}-\rho_{\pi}\right)}{\left(e_{1}-\rho_{\pi}\right)\left(e_{2}-\rho_{\pi}\right)(1-\beta \phi)} \\
=\frac{\beta^{2}\left(e_{2}-e_{3}\right)\left(e_{2}-\rho_{\pi}\right)-\left(1-\beta e_{2}\right)^{2}-(\beta+\kappa-1)\left(1-\beta e_{2}\right)}{\beta\left(e_{1}-\rho_{\pi}\right)\left(e_{2}-\rho_{\pi}\right)(1-\beta \phi)} \\
=\frac{\beta^{2}\left(e_{2}^{2}-\left(e_{3}+\rho_{\pi}\right) e_{2}+\rho_{\pi} e_{3}\right)-\left(\beta^{2} e_{2}^{2}-2 \beta e_{2}+1\right)-(\beta+\kappa-1)+(\beta+\kappa-1) \beta e_{2}}{\beta\left(e_{1}-\rho_{\pi}\right)\left(e_{2}-\rho_{\pi}\right)(1-\beta \phi)} \\
=\frac{\beta^{2}\left(\rho_{\pi} e_{3}-\left(e_{3}+\rho_{\pi}\right) e_{2}\right)+2 \beta e_{2}-(\beta+\kappa)+(\beta+\kappa-1) \beta e_{2}}{\beta\left(e_{1}-\rho_{\pi}\right)\left(e_{2}-\rho_{\pi}\right)(1-\beta \phi)} \\
=\frac{\left[\beta^{2}\left(1-e_{3}\right)+\beta\left(1-\beta \rho_{\pi}\right)+\beta \kappa\right] e_{2}+\beta^{2} \rho_{\pi} e_{3}-(\beta+\kappa)}{\beta\left(e_{1}-\rho_{\pi}\right)\left(e_{2}-\rho_{\pi}\right)(1-\beta \phi)} .
\end{gathered}
$$

The denominator is unambiguously positive for all parameter values under PMAF, and $\Theta$ will therefore be positive if and only if the numerator is also positive. Note that the numerator is a linear and increasing function of $e_{2}$ because the slope, $\left[\beta^{2}\left(1-e_{3}\right)+\beta\left(1-\beta \rho_{\pi}\right)+\beta \kappa\right]$, is positive. This implies that $\Theta>0$ for sufficiently large $e_{2}$ - or sufficiently small $\psi$. It is straightforward to show that $\Theta>0$ if and only if $-\infty<\psi<\bar{\psi}^{*}$ where:

$$
\bar{\psi}^{*} \equiv 1-\frac{(\beta+\kappa)-\beta^{2} \rho_{\pi} e_{3}}{\beta\left(1-e_{3}\right)+\left(1-\beta \rho_{\pi}\right)+\kappa}=1-\frac{\beta+\kappa-\beta^{2} \rho_{\pi} e_{3}}{\beta\left(e_{1}-\rho_{\pi}\right)}
$$


It remains to show that $\bar{\psi}^{*}$ is positive. Note that the denominator of $\bar{\psi}^{*}$ is positive. Consider the numerator, $g(\phi) \equiv \beta\left(e_{1}-\rho_{\pi}\right)-\left(\beta+\kappa-\beta^{2} \rho_{\pi} e_{3}\right)$. Given other parameters, $g(\phi)$ has the smallest value at $\phi=1$ because $g^{\prime}(\phi)<0$. Evaluate $g(\phi)$ at $\phi=1$ :

$$
\begin{aligned}
g(1) & =\beta\left(e_{1}-\rho_{\pi}\right)-\left(\beta+\kappa-\beta^{2} \rho_{\pi} e_{3}\right)=\beta\left(\frac{\kappa+1}{\beta}-\rho_{\pi}\right)-\left(\beta+\kappa-\beta^{2} \rho_{\pi}\right) \\
& =(1-\beta)\left(1-\beta \rho_{\pi}\right)>0,
\end{aligned}
$$

which implies $\bar{\psi}^{*}>0$. Finally, redefining $\bar{\psi}^{*}$ as $\bar{\psi}^{*} \equiv \min \left\{\bar{\psi}^{*}, \bar{\psi}\right\}$, we establish that:

$$
0<\bar{\psi}^{*} \leq \bar{\psi} \equiv 1-\beta
$$

In addition, we can show that $\bar{\psi}^{*}$ depends crucially on the slope of the Phillips curve and satisfies:

$$
\begin{aligned}
& \lim _{\kappa \rightarrow \infty} \bar{\psi}^{*}=0 \\
& \lim _{\kappa \rightarrow 0} \bar{\psi}^{*}=1-\beta .
\end{aligned}
$$

Therefore, the interval of $\psi$ that makes $\Phi$ positive - i.e. that makes the coefficient on $\pi_{t}^{*}$ negative always contains zero (i.e. always includes the benchmark FTPL case). In addition, the interval covers the AF region $(-\infty<\psi<\bar{\psi})$ almost entirely. The interval coincides exactly with the entire AF region when prices are fully sticky (i.e. when $\kappa=0$ ). In other cases, the upper bound for positive $\Phi, \bar{\psi}^{*}$, is positive, but may be slightly below the upper bound for $\mathrm{AF}, \bar{\psi}$.

(II) It is trivial to show $\Gamma(\phi, \psi)>0$ because $e_{1}>1>\rho_{r}$ and $e_{2}>1>\rho_{r}$ under PMAF.

(III) It is trivial to show $\Omega(\phi, \psi)=\frac{\beta\left(e_{2}-e_{3}\right)}{\bar{b}(1-\beta \phi)}>0$ because $e_{2}>1>e_{3}$ and $1>\beta \phi$ under PMAF. (Also see Lemma 1)

\section{Proof of Proposition 4}

(I) To show $\frac{\partial \Phi(\phi, \psi)}{\partial \phi}>0$, let us first consider $\frac{\partial \Theta(\phi, \psi)}{\partial \phi}$ :

$$
\begin{aligned}
\frac{\partial \Theta(\phi, \psi)}{\partial \phi} & =\frac{\left\{\begin{array}{c}
\frac{\partial \Omega}{\partial \phi} \bar{b}\left(e_{1}-\rho_{\pi}\right)^{2}\left(e_{2}-\rho_{\pi}\right)+\frac{\partial e_{1}}{\partial \phi} \Omega \bar{b}\left(e_{1}-\rho_{\pi}\right)\left(e_{2}-\rho_{\pi}\right) \\
-\frac{\partial e_{1}}{\partial \phi}\left(e_{2}-\rho_{\pi}\right)\left[\Omega \bar{b}\left(e_{1}-\rho_{\pi}\right)-\kappa \beta^{-1}\right.
\end{array}\right]}{\left(e_{1}-\rho_{\pi}\right)^{2}\left(e_{2}-\rho_{\pi}\right)^{2}} \\
& =\frac{\frac{\partial \Omega}{\partial \phi} \bar{b}\left(e_{1}-\rho_{\pi}\right)^{2}\left(e_{2}-\rho_{\pi}\right)-\frac{\partial e_{3}}{\partial \phi}\left(e_{2}-\rho_{\pi}\right) \kappa \beta^{-1}}{\left(e_{1}-\rho_{\pi}\right)^{2}\left(e_{2}-\rho_{\pi}\right)^{2}}=\frac{\frac{\partial \Omega}{\partial \phi} \bar{b}\left(e_{1}-\rho_{\pi}\right)^{2}-\frac{\partial e_{3}}{\partial \phi} \kappa \beta^{-1}}{\left(e_{1}-\rho_{\pi}\right)^{2}\left(e_{2}-\rho_{\pi}\right)}
\end{aligned}
$$

Now let us take the partial derivative of $\Phi(\phi, \psi)$ with respect to $\phi$ :

$$
\begin{aligned}
\frac{\partial \Phi(\phi, \psi)}{\partial \phi} & \equiv \Theta(\phi, \psi)+\phi \frac{\partial \Theta(\phi, \psi)}{\partial \phi} \\
& =\frac{\Omega \bar{b}\left(e_{1}-\rho_{\pi}\right)-\kappa \beta^{-1}}{\left(e_{1}-\rho_{\pi}\right)\left(e_{2}-\rho_{\pi}\right)}+\phi \frac{\frac{\partial \Omega}{\partial \phi} \bar{b}\left(e_{1}-\rho_{\pi}\right)^{2}-\frac{\partial e_{3}}{\partial \phi} \kappa \beta^{-1}}{\left(e_{1}-\rho_{\pi}\right)^{2}\left(e_{2}-\rho_{\pi}\right)} \\
& =\frac{\frac{\beta\left(e_{2}-e_{3}\right)}{(1-\beta \phi)}\left(e_{1}-\rho_{\pi}\right)^{2}-\left(e_{1}-\rho_{\pi}\right) \kappa \beta^{-1}+\phi \frac{\partial \Omega}{\partial \phi} \bar{b}\left(e_{1}-\rho_{\pi}\right)^{2}-\phi \frac{\partial e_{3}}{\partial \phi} \kappa \beta^{-1}}{\left(e_{1}-\rho_{\pi}\right)^{2}\left(e_{2}-\rho_{\pi}\right)}
\end{aligned}
$$


Since the denominator is positive, $\frac{\partial \Phi(\phi, \psi)}{\partial \phi}>0$ if and only if the numerator is positive - that is,

$$
\begin{gathered}
0<\frac{\partial \Phi(\phi, \psi)}{\partial \phi} \Longleftrightarrow \\
0<\frac{\beta\left(e_{2}-e_{3}\right)}{(1-\beta \phi)}\left(e_{1}-\rho_{\pi}\right)^{2}-\left(e_{1}-\rho_{\pi}\right) \kappa \beta^{-1}+\phi \frac{\partial \Omega}{\partial \phi} \bar{b}\left(e_{1}-\rho_{\pi}\right)^{2}-\phi \frac{\partial e_{3}}{\partial \phi} \kappa \beta^{-1} \\
\Longleftrightarrow \frac{\beta\left(e_{2}-e_{3}\right)}{(1-\beta \phi)}+\phi \frac{\partial \Omega}{\partial \phi} \bar{b} \geq \frac{\phi \frac{\partial e_{3}}{\partial \phi} \kappa \beta^{-1}+\left(e_{1}-\rho_{\pi}\right) \kappa \beta^{-1}}{\left(e_{1}-\rho_{\pi}\right)^{2}} \\
\Longleftrightarrow \frac{\beta\left(e_{2}-e_{3}\right)}{(1-\beta \phi)}+\phi \beta \frac{\beta e_{2}-\beta e_{3}-(1-\beta \phi) \frac{\partial e_{3}}{\partial \phi}}{\phi \frac{\partial e_{3}}{\partial \phi} \kappa \beta^{-1}+\left(e_{1}-\rho_{\pi}\right) \kappa \beta^{-1}} \\
\Longleftrightarrow \beta\left(e_{2}-e_{3}\right) \frac{1-\beta \phi)^{2}}{(1-\beta \phi)^{2}}>\frac{\phi \frac{\partial e_{3}}{\partial \phi} \kappa \beta^{-1}+\left(e_{1}-\rho_{\pi}\right) \kappa \beta^{-1}}{\left(e_{1}-\rho_{\pi}\right)^{2}}+\frac{\beta \phi}{(1-\beta \phi)} \frac{\partial e_{3}}{\partial \phi} \\
\Longleftrightarrow \beta e_{2}>\beta e_{3}+(1-\beta \phi)^{2}\left\{\frac{\phi \frac{\partial e_{3}}{\partial \phi} \kappa \beta^{-1}+\left(e_{1}-\rho_{\pi}\right) \kappa \beta^{-1}}{\left(e_{1}-\rho_{\pi}\right)^{2}} \frac{\beta \phi}{(1-\beta \phi)} \frac{\partial e_{3}}{\partial \phi}\right\} \\
\Longleftrightarrow \psi<1-\left[\beta e_{3}+(1-\beta \phi)^{2}\left\{\frac{\phi \frac{\partial e_{3}}{\partial \phi} \kappa \beta^{-1}+\left(e_{1}-\rho_{\pi}\right) \kappa \beta^{-1}}{\left(e_{1}-\rho_{\pi}\right)^{2}}+\frac{\beta \phi}{(1-\beta \phi)} \frac{\partial e_{3}}{\partial \phi}\right\}\right] \\
\left.\qquad \psi(1-\beta \phi)^{2}\left\{\frac{\kappa \beta^{-1}}{\left(e_{1}-\rho_{\pi}\right)}+\frac{\phi \kappa \beta^{-1}}{\left(e_{1}-e_{3}\right)}\left[\frac{\kappa \beta^{-1}}{\left(e_{1}-\rho_{\pi}\right)^{2}}+\frac{\beta}{(1-\beta \phi)}\right]\right\}\right]
\end{gathered}
$$

In sum,

$$
\frac{\partial \Phi(\phi, \psi)}{\partial \phi}>0 \Longleftrightarrow \psi<\bar{\psi}^{* *}
$$

where

$$
\bar{\psi}^{* *} \equiv 1-\left[\beta e_{3}+(1-\beta \phi)^{2}\left\{\frac{\kappa \beta^{-1}}{\left(e_{1}-\rho_{\pi}\right)}+\frac{\phi \kappa \beta^{-1}}{\left(e_{1}-e_{3}\right)}\left[\frac{\kappa \beta^{-1}}{\left(e_{1}-\rho_{\pi}\right)^{2}}+\frac{\beta}{(1-\beta \phi)}\right]\right\}\right]
$$

As before, it remains to show that $\bar{\psi}^{* *}$ is positive. First, suppose $\bar{\psi}^{* *} \geq \bar{\psi}^{*}$ or $\bar{\psi}^{* *} \geq \bar{\psi}^{* * *}$. We have already shown $\bar{\psi}^{*}>0$ above, and it will be shown $\bar{\psi}^{* * *}>0$ below. Thus, it must be that $\bar{\psi}^{* *}>0$. Suppose instead $\bar{\psi}^{* *} \leq \bar{\psi}^{*}$ and $\bar{\psi}^{* *} \leq \bar{\psi}^{* * *}$. In this case, if $\psi<\bar{\psi}^{* *}$, then $\Theta(\phi, \psi)>0$ and $\frac{\partial \Omega(\phi, \psi)}{\partial \phi}>0$. But, we can show from $(26)$ that $\frac{\partial \Theta(\phi, \psi)}{\partial \phi}>0$ at $\psi=0$ if $\frac{\partial \Omega(\phi, \psi)}{\partial \phi}>0$, which implies $\frac{\partial \Phi(\phi, \psi)}{\partial \phi}>0$ at $\psi=0$. Since $\psi<\bar{\psi}^{* *}$ is the sufficient and necessary condition for $\frac{\partial \Phi(\phi, \psi)}{\partial \phi}>0$, it should always contain zero. Therefore, $\bar{\psi}^{* *}>0$. Finally, redefining $\bar{\psi}^{* *}$ as $\bar{\psi}^{* *} \equiv \min \left\{\bar{\psi}^{* *}, \bar{\psi}\right\}$, we establish that:

$$
0<\bar{\psi}^{* *} \leq \bar{\psi} \equiv 1-\beta
$$

In addition, similar to $\bar{\psi}^{*}$, it can be shown that:

$$
\begin{aligned}
\lim _{\kappa \rightarrow \infty} \bar{\psi}^{* *} & =0 \\
\lim _{\kappa \rightarrow 0} \bar{\psi}^{* *} & =1-\beta .
\end{aligned}
$$

(II) Showing $\frac{\partial \Gamma(\phi, \psi)}{\partial \phi}>0$ is trivial because $\frac{\partial e_{1}}{\partial \phi}<0$ and $e_{2}>1$ under PMAF. 
(III) To show $\frac{\partial \Omega(\phi, \psi)}{\partial \phi}>0$, take the partial derivative of $\Omega(\phi, \psi)$ with respect to $\phi$ :

$$
\frac{\partial \Omega}{\partial \phi}=\frac{\beta}{\bar{b}} \frac{\beta e_{2}-\beta e_{3}-(1-\beta \phi) \frac{\partial e_{3}}{\partial \phi}}{(1-\beta \phi)^{2}}=\frac{\beta}{\bar{b}} \frac{h(\phi)}{(1-\beta \phi)^{2}},
$$

where

$$
h(\phi) \equiv \beta e_{2}-\beta e_{3}-(1-\beta \phi) \frac{\partial e_{3}}{\partial \phi} .
$$

Since $\frac{\beta}{b} \frac{1}{(1-\beta \phi)^{2}}$ is clearly positive, $\frac{\partial \Omega}{\partial \phi}$ and $h(\phi)$ must have the same sign. Note that

$$
\begin{gathered}
h(\phi)>0 \Longleftrightarrow \beta e_{2}>\beta e_{3}+(1-\beta \phi) \frac{\partial e_{3}}{\partial \phi} \\
\Longleftrightarrow \beta e_{2}>\beta e_{3}+(1-\beta \phi) \frac{\kappa}{\beta+\kappa+1-2 \beta e_{3}} \quad\left(\because \frac{\partial e_{3}}{\partial \phi}=\frac{\kappa}{\beta+\kappa+1-2 \beta e_{3}}>0\right) \\
\Longleftrightarrow \beta e_{2}>\beta e_{3}+(1-\beta \phi) \frac{\kappa}{\beta\left(e_{1}-e_{3}\right)} \\
\Longleftrightarrow \beta e_{2}>\frac{\beta^{2} e_{1} e_{3}-\beta^{2} e_{3}^{2}+(1-\beta \phi) \kappa}{\beta\left(e_{1}-e_{3}\right)} \\
\Longleftrightarrow \psi<e_{2}>\frac{\beta+\kappa-\beta^{2} e_{3}^{2}}{\beta\left(e_{1}-e_{3}\right)} \\
\underbrace{1-\frac{\beta+\kappa-\beta^{2} e_{3}^{2}}{\beta\left(e_{1}-e_{3}\right)}}_{\equiv \bar{\psi}^{* * *}}=\frac{\beta\left(e_{1}-e_{3}\right)-\left(\beta+\kappa-\beta^{2} e_{3}^{2}\right)}{\beta\left(e_{1}-e_{3}\right)}
\end{gathered}
$$

Once again, it remains to show that $\bar{\psi}^{* * *}$ is positive. Consider the numerator of $\bar{\psi}^{* * *}, g(\phi) \equiv$ $\beta\left(e_{1}-e_{3}\right)-\left(\beta+\kappa-\beta^{2} e_{3}^{2}\right)$. Given other parameters, $g(\phi)$ has the smallest value at $\phi=1$ because $g^{\prime}(\phi)<0$. Evaluate $g(\phi)$ at $\phi=1$ :

$$
\begin{aligned}
g(1) & =\beta\left(e_{1}-e_{3}\right)-\left(\beta+\kappa-\beta^{2} e_{3}^{2}\right)=\beta\left(\frac{\kappa+1}{\beta}-1\right)-\left(\beta+\kappa-\beta^{2}\right) \\
& =(1-\beta)^{2}>0 .
\end{aligned}
$$

which implies $\bar{\psi}^{* * *}>0$. Finally, redefining $\bar{\psi}^{* * *}$ as $\bar{\psi}^{* * *} \equiv \min \left\{\bar{\psi}^{* * *}, \bar{\psi}\right\}$, we establish that:

$$
0<\bar{\psi}^{* * *} \leq \bar{\psi} \equiv 1-\beta
$$

In addition, similar to $\bar{\psi}^{*}$ and $\bar{\psi}^{* *}$, it can be shown that:

$$
\begin{aligned}
\lim _{\kappa \rightarrow \infty} \bar{\psi}^{* * *} & =0 \\
\lim _{\kappa \rightarrow 0} \bar{\psi}^{* * *} & =1-\beta .
\end{aligned}
$$

\section{Proof of Proposition 5}

(I) To show $\frac{\partial \Phi(\phi, \psi)}{\partial \psi}<0$ under PMAF, take the partial derivative of $\Theta=\frac{\beta\left(e_{2}-e_{3}\right)\left(e_{1}-\rho_{\pi}\right)-\kappa \beta^{-1}(1-\beta \phi)}{\left(e_{1}-\rho_{\pi}\right)\left(e_{2}-\rho_{\pi}\right)(1-\beta \phi)}$ with 
respect to $e_{2}$ :

$$
\begin{gathered}
\frac{\partial \Theta}{\partial e_{2}}=\frac{\beta\left(e_{1}-\rho_{\pi}\right)^{2}\left(e_{2}-\rho_{\pi}\right)(1-\beta \phi)-\left(e_{1}-\rho_{\pi}\right)(1-\beta \phi)\left[\beta\left(e_{2}-e_{3}\right)\left(e_{1}-\rho_{\pi}\right)-\kappa \beta^{-1}(1-\beta \phi)\right]}{\left[\left(e_{1}-\rho_{\pi}\right)\left(e_{2}-\rho_{\pi}\right)(1-\beta \phi)\right]^{2}} \\
=\frac{\left(e_{1}-\rho_{\pi}\right)(1-\beta \phi)\left[\beta\left(e_{1}-\rho_{\pi}\right)\left(e_{2}-\rho_{\pi}\right)-\beta\left(e_{2}-e_{3}\right)\left(e_{1}-\rho_{\pi}\right)+\kappa \beta^{-1}(1-\beta \phi)\right]}{\left[\left(e_{1}-\rho_{\pi}\right)\left(e_{2}-\rho_{\pi}\right)(1-\beta \phi)\right]^{2}} \\
=\frac{\left(e_{1}-\rho_{\pi}\right)(1-\beta \phi)}{\left[\left(e_{1}-\rho_{\pi}\right)\left(e_{2}-\rho_{\pi}\right)(1-\beta \phi)\right]^{2}} g
\end{gathered}
$$

where

$$
g \equiv \beta\left(e_{1}-\rho_{\pi}\right)\left(e_{2}-\rho_{\pi}\right)-\beta\left(e_{2}-e_{3}\right)\left(e_{1}-\rho_{\pi}\right)+\kappa \beta^{-1}(1-\beta \phi) .
$$

Since $\frac{\left(e_{1}-\rho_{\pi}\right)(1-\beta \phi)}{\left[\left(e_{1}-\rho_{\pi}\right)\left(e_{2}-\rho_{\pi}\right)(1-\beta \phi)\right]^{2}}>0$, we focus on $g$, which can be written as:

$$
g=\beta\left[\rho_{\pi}^{2}-\left(e_{1}+e_{3}\right) \rho_{\pi}+e_{1} e_{3}\right]+\kappa \beta^{-1}(1-\beta \phi) .
$$

Note that

$$
\begin{aligned}
e_{1}+e_{3} & =\frac{\beta+\kappa+1}{\beta} \\
e_{1} e_{3} & =\frac{\kappa \phi+1}{\beta} .
\end{aligned}
$$

Using these, rewrite $g$ and regard $g$ as a function of $\rho_{\pi} \in(0,1)$ given other parameters:

$$
g\left(\rho_{\pi}\right)=\beta \rho_{\pi}^{2}-(\beta+\kappa+1) \rho_{\pi}+1+\kappa \beta^{-1}
$$

Note that $g\left(\rho_{\pi}\right)$ is a convex and quadratic function of $\rho_{\pi}$, and

$$
g(0)=1+\kappa \beta^{-1}>g(1)=\kappa\left(\beta^{-1}-1\right)>0 .
$$

Moreover,

$$
g^{\prime}(0)<0 \text { and } g^{\prime}(1)<0 .
$$

Therefore, it must be that $g>0$ for $\rho_{\pi} \in(0,1), \beta \in(0,1)$ and $\kappa \in(0, \infty)$. Hence,

$$
\frac{\partial \Theta}{\partial e_{2}}=\frac{\left(e_{1}-\rho_{\pi}\right)(1-\beta \phi)}{\left[\left(e_{1}-\rho_{\pi}\right)\left(e_{2}-\rho_{\pi}\right)(1-\beta \phi)\right]^{2}} g>0 .
$$

This implies that

$$
\frac{\partial \Theta}{\partial \psi}<0 \text { and } \frac{\partial \Phi}{\partial \psi}<0
$$

because $e_{2}$ is decreasing in $\psi$.

(II) Showing $\frac{\partial \Gamma(\phi, \psi)}{\partial \psi}>0$ is trivial because $e_{1}>1$ and $\frac{\partial e_{2}}{\partial \psi}<0$ under PMAF.

(III) Showing $\frac{\partial \Omega(\phi, \psi)}{\partial \psi}$ is also trivial as $\frac{\partial e_{2}}{\partial \psi}<0$.

Discussion on $\bar{\psi}^{*}, \bar{\psi}^{* *}, \bar{\psi}^{* * *}$ and $\bar{\psi} \equiv 1-\beta$. Although the upper bounds, $\bar{\psi}^{*}, \bar{\psi}^{* *}$ and $\bar{\psi}^{* * *}$ generally have different values, they converge to the same numbers as the slope of NKPC approaches to 
infinity and zero. In particular, all of them equal $\bar{\psi} \equiv 1-\beta$ - the "true" upper bound for AF - when $\kappa=0$. However, $\bar{\psi}^{*}, \bar{\psi}^{* *}, \bar{\psi}^{* * *}$ and $\bar{\psi}$ would be indistinguishable in practice because $\bar{\psi}^{*}, \bar{\psi}^{* *}, \bar{\psi}^{* * *} \in(0, \bar{\psi}]$ and $\bar{\psi}$ would have a tiny value.

\section{B.4.3 Proof under PMPF}

Proof of Proposition 6 To prove $\Gamma(\phi, \psi)>0$ and $\Phi(\phi, \psi)=\phi \Gamma(\phi, \psi) \geq 0$ (the equality holds at $\phi=0)$, we show that

$$
\Gamma(\phi, \psi)=\frac{q_{11}}{d^{2}}\left[\beta^{-1}\left(q_{11}-q_{12}\right)-\phi q_{11}\right]
$$

is always positive. Note that

$$
\begin{aligned}
v_{23} & =\frac{-\beta e_{3}-(\psi-1)}{\bar{b}(1-\beta \phi)}, \\
v_{13} & =\frac{\beta e_{3}(\kappa-1+\psi+\beta)-(\beta+\psi+\beta \kappa \phi-1)}{\bar{b} \kappa(1-\beta \phi)}, \\
q_{11} & =\frac{v_{23}}{v_{11} v_{23}-v_{13} v_{21}}, \\
q_{12} & =\frac{-v_{13}}{v_{11} v_{23}-v_{13} v_{21}} .
\end{aligned}
$$

Then, (27) can be written as:

$$
\Gamma(\phi, \psi)=\frac{v_{23}\left[\left(\beta^{-1}-\phi\right) v_{23}+\beta^{-1} v_{13}\right]}{d^{2}\left(v_{11} v_{23}-v_{13} v_{21}\right)^{2}} .
$$

Note that $v_{13}=v_{23}=0$ at $\psi=1-\beta e_{3}$, and

$$
\frac{\partial v_{23}}{\partial \psi}<0 \text { and } \frac{\partial v_{13}}{\partial \psi}<0,
$$

which implies that $v_{13}$ and $v_{23}$ have the same sign for any values of $\psi$ given other parameters. This proves that (28) is always positive since $\beta^{-1}>1>\phi$ under PMPF, and consequently $\Phi(\phi, \psi)=\phi \Gamma(\phi, \psi) \geq 0$.

Finally, it is clear from the solution that government debt is not a state variable for inflation. It is straightforward to show that government debt is not a state variable for output as well.

\section{B.4.4 A special case with repeated eigenvalues}

In the proofs above, we implicitly assume that the matrix $G$ has distinct eigenvalues. There is however one special case in which $G$ has repeated eigenvalues and hence is not diagonalizable. Such case arises when $\psi=1-\beta e_{3}$ and only under PMPF. ${ }^{33}$ In this case, one should use the Jordan decomposition - instead of the eigenvalue decomposition - as $G=W J W^{-1}$ where

$$
J=\left(\begin{array}{ccc}
e_{1} & 0 & 0 \\
0 & e_{3} & 1 \\
0 & 0 & e_{3}
\end{array}\right) \text { and } W=\left(\begin{array}{ccc}
w_{11} & 0 & w_{13} \\
w_{21} & 0 & w_{23} \\
w_{31} & w_{32} & w_{33}
\end{array}\right)
$$

\footnotetext{
${ }^{33}$ This special case does not arise under AMPF and PMAF. Under AMPF, $e_{3}>1$, and fiscal policy is active at $\psi=1-\beta e_{3}$. Under PMAF, on the other hand, $0<e_{3}<1$, and fiscal policy is passive at $\psi=1-\beta e_{3}$.
} 
with

$$
\begin{aligned}
& e_{1}=\frac{1}{2 \beta}\left(\beta+\kappa+1+\sqrt{(\beta+\kappa+1)^{2}-4 \beta(1+\kappa \phi)}\right) \\
& e_{3}=\frac{1}{2 \beta}\left(\beta+\kappa+1-\sqrt{(\beta+\kappa+1)^{2}-4 \beta(1+\kappa \phi)}\right) .
\end{aligned}
$$

Note that the eigenvalues are identical to the two of the three eigenvalues in the previous sections. Note that

$$
W^{-1}=\left(\begin{array}{ccc}
1 & z_{12} & 0 \\
0 & -\beta \kappa^{-1} & z_{23} \\
1 & z_{32} & 0
\end{array}\right) .
$$

In particular,

$$
z_{12}=-\frac{\psi}{\kappa}<0
$$

Then the unstable part of the system is the same as before except that $q_{11}$ and $q_{12}$ are now replaced by 1 and $z_{12}$, respectively. Therefore

$$
\begin{aligned}
\Gamma(\phi, \psi) & =\frac{1}{d^{2}}\left[\beta^{-1}\left(1-z_{12}\right)-\phi\right]=\frac{1}{d^{2}}\left[\left(\beta^{-1}-\phi\right)-\beta^{-1} z_{12}\right]>0 \\
\Phi(\phi, \psi) & =\phi \Gamma(\phi, \psi) \geq 0 .
\end{aligned}
$$

In addition, following the same steps as before, one can easily show that $b_{t-1}$ does not directly affect the dynamics of inflation and output expectation.

\section{Model of Fiscal Dominance}

We make only two deviations - that are necessary for our purpose - from the model we considered above to illustrate the mechanism behind "Unpleasant Monetarist Arithmetic." First, we introduce money as an argument of household utility function following the money-in-the-utility (MIU) specification. Second, we specify monetary policy rule differently. Specifically, the interest rate rule is replaced by a seignorage rule in which under fiscal dominance, the monetary authority increases seignorage in response to an increase in the outstanding value of government debt.$^{34}$

\section{C.1 Households}

Identical households choose sequences of $\left\{C_{t}, B_{t}, M_{t}, N_{t}, D_{t+1}\right\}$ to maximize

$$
E_{0} \sum_{t=0}^{\infty} \beta^{t}\left[\log C_{t}+\varrho \frac{\left(\frac{M_{t}}{P_{t}}\right)^{1-\vartheta}}{1-\vartheta}-\frac{N_{t}^{1+\varphi}}{1+\varphi}\right]
$$

\footnotetext{
${ }^{34}$ To make this new model as close as possible to our baseline model, we still assume that the government issues nominal debt unlike Sargent and Wallace (1981). We can instead assume that the debt is real, which will be inconsequential for our results.
} 
subject to

$$
P_{t} C_{t}+B_{t}+M_{t}+E_{t}\left[Q_{t, t+1} D_{t+1}\right]=R_{t-1} B_{t-1}+M_{t-1}+D_{t}+W_{t} N_{t}+\Pi_{t}-P_{t} \tau_{t}
$$

where $C_{t}$ is consumption, $\frac{M_{t}}{P_{t}}$ is real balances, $N_{t}$ is labor hours, $P_{t}$ is the price level, $B_{t}$ is the amount of one-period risk-less nominal government bond, $R_{t}$ is the gross nominal interest rate, $W_{t}$ is the nominal wage rate, $\Pi_{t}$ is profits of intermediate firms, and $\tau_{t}$ is government taxes net of transfers. The parameter, $\varphi \geq 0$, denotes the inverse of the Frisch elasticity of labor supply while $\varrho$ and $\vartheta$ are related to money demand. In addition to the government bond, households trade at time $t$ one-period state-contingent nominal securities $D_{t+1}$ at price $Q_{t, t+1}$.

\section{C.2 Firms}

The set-up for firms is identical to that of the baseline simple model.

\section{C.3 Government}

Each period, the government collects lump-sum taxes $\tau_{t}$, supplies money $M_{t}$, and issues one-period nominal bonds $B_{t}$ to finance its consumption $G_{t}$, and interest payments. Accordingly, the flow budget constraint is given by:

$$
\frac{B_{t}}{P_{t}}+\frac{M_{t}}{P_{t}}=R_{t-1} \frac{B_{t-1}}{P_{t}}+\frac{M_{t-1}}{P_{t}}+G_{t}-\tau_{t}
$$

For simplicity, we assume $G_{t}=0$, which is inconsequential for our theoretical results. The flow budget constraint can be rewritten as:

$$
R_{t}^{-1} b_{t}=b_{t-1} \frac{1}{\pi_{t}}-\tau_{t}-s_{t}
$$

where $b_{t} \equiv R_{t} \frac{B_{t}}{P_{t}}$ denotes the real maturity value of government debt and $s_{t} \equiv\left(M_{t}-M_{t-1}\right) / P_{t}$ is seignorage.

Monetary and fiscal policies are described by simple rules:

$$
\begin{gathered}
s_{t}=\xi_{b} b_{t-1}+\varepsilon_{s, t}, \\
\frac{\tau_{t}}{\bar{\tau}}=\left(\frac{b_{t-1}}{\bar{b}}\right)^{\psi},
\end{gathered}
$$

where $\bar{\tau}$ and $\bar{b}$ are the steady state value of $\tau_{t}$ and $b_{t}$, respectively, and $\varepsilon_{s, t}$ is an exogenous shock to the seignorage. Importantly, given the monetary policy rule, the central bank's behavior could be dictated by two (potentially conflicting) objectives: price stability and fiscal sustainability. Modeling monetary policy as an exogenous path related to money supply is in the spirit of Sargent and Wallace (1981). Here, we allow seigniorage to respond to outstanding debt in order to satisfy the government budget constraint when taxes do not fully back-up debt. Such a case would constitute fiscal dominance.

\section{C.4 Approximate Model}

We log-linearize the equilibrium conditions around the non-stochastic steady state with values $\{\bar{\pi}, \bar{Y}, \bar{R}, \bar{b}, \bar{\tau}, \bar{m}$, $\bar{s}$. Again, we assume that inflation is zero in the steady state. Since the log-linearized model is standard, we omit a detailed derivation. Let us denote by $\hat{X}_{t}$ the $\log$ deviation of a variable $X_{t}$ from its steady state $\bar{X}$ 
$\left(\hat{X}_{t}=\ln X_{t}-\ln \bar{X}\right)$, except for three variables, $\hat{b}_{t}, \hat{\tau}_{t}$ and $\hat{s}_{t}$. Following Woodford (2003), we define $\hat{b}_{t}, \hat{\tau}_{t}$ and $\hat{s}_{t}$ as a deviation of $b_{t}, \tau_{t}$, and $s_{t}$ from the corresponding steady state values, measured as a ratio of steady-state output: $\hat{b}_{t}=\left(b_{t}-\bar{b}\right) / \bar{Y}, \hat{\tau}_{t}=\left(\tau_{t}-\bar{\tau}\right) / \bar{Y}$, and $\hat{s}_{t}=\left(s_{t}-\bar{s}\right) / \bar{Y}$. The approximate model is characterized by the following seven equations:

$$
\begin{aligned}
\hat{m}_{t} & =\eta_{y} \hat{Y}_{t}-\eta_{i} \hat{R}_{t} \\
\hat{Y}_{t} & =E_{t} \hat{Y}_{t+1}-\left(\hat{R}_{t}-E_{t} \hat{\pi}_{t+1}\right), \\
\hat{\pi}_{t} & =\kappa \hat{Y}_{t}+\beta E_{t} \hat{\pi}_{t+1}, \\
\hat{s}_{t} & =\xi_{b} \hat{b}_{t-1}+\varepsilon_{s, t}, \\
\hat{\tau}_{t} & =\psi \hat{b}_{t-1}, \\
\hat{b}_{t} & =\beta^{-1} \hat{b}_{t-1}+\bar{b} \hat{R}_{t}-\beta^{-1} \bar{b} \hat{\pi}_{t}-\beta^{-1} \hat{\tau}_{t}-\beta^{-1} \hat{s}_{t}, \\
\hat{s}_{t} & =\bar{m}\left(\hat{m}_{t}-\hat{m}_{t-1}+\hat{\pi}_{t}\right),
\end{aligned}
$$

where $\kappa=(1-\alpha)(1-\alpha \beta) / \alpha, \eta_{y}=1 / \vartheta, \eta_{i}=\beta /[(1-\beta) \vartheta]$, and $\bar{m}=\varrho(1-\beta)$. Note that we focus on a case where $\bar{b}=0$ to make the distinction from the PMAF policy regime stark.

\section{C.5 Equilibrium and Model Solution}

One can reduce the model to a dynamic system of $\left\{\hat{Y}_{t}, \hat{\pi}_{t}, \hat{m}_{t}, \hat{b}_{t}\right\}$ :

$$
\begin{aligned}
\left(1+\eta_{y} \eta_{i}^{-1}\right) \hat{Y}_{t} & =E_{t} \hat{Y}_{t+1}+\eta_{i}^{-1} \hat{m}_{t}+E_{t} \hat{\pi}_{t+1}, \\
\hat{\pi}_{t} & =\kappa \hat{Y}_{t}+\beta E_{t} \hat{\pi}_{t+1}, \\
\hat{m}_{t} & =\frac{\xi_{b}}{\bar{m}} \hat{b}_{t-1}+\hat{m}_{t-1}-\hat{\pi}_{t}+\frac{1}{\bar{m}} \varepsilon_{s, t}, \\
\hat{b}_{t} & =\beta^{-1}\left[1-\left(\psi+\xi_{b}\right)\right] \hat{b}_{t-1}-\beta^{-1} \varepsilon_{s, t} .
\end{aligned}
$$

For simplicity, we assume that $\varepsilon_{s, t}$ is independent over time. Since it is difficult to derive a closed-form solution for this model, we investigate the properties of its solution by reducing the number of endogenous variables. Let $\rho_{b}=\beta^{-1}\left[1-\left(\psi+\xi_{b}\right)\right]$. There does not exist any stable solution if $\left|\rho_{b}\right| \geq 1$.

Let us assume that $\left|\rho_{b}\right|<1$. Then, there exists a solution for $\hat{b}_{t}$ regardless of the values of other parameters. Note that from the seigniorage rule (32),

$$
\hat{\pi}_{t}=\frac{\xi_{b}}{\bar{m}} \hat{b}_{t-1}-\hat{m}_{t}+\hat{m}_{t-1}+\frac{1}{\bar{m}} \varepsilon_{s, t}
$$

which can be substituted for $\hat{\pi}_{t}$ in (31) as

$$
\kappa \hat{Y}_{t}=\frac{\xi_{b}}{\bar{m}}\left(\hat{b}_{t-1}-\beta \hat{b}_{t}\right)-\left(\hat{m}_{t}-\beta E_{t} \hat{m}_{t+1}\right)+\left(\hat{m}_{t-1}-\beta \hat{m}_{t}\right)+\frac{1}{\bar{m}}\left(1-\beta \rho_{s}\right) \varepsilon_{s, t} .
$$

Now use (34) and (35) to eliminate $\hat{Y}_{t}$ and $\hat{\pi}_{t}$ in (30) and arrange terms to get

$$
\begin{aligned}
& \beta \kappa^{-1} E_{t} \hat{m}_{t+2}-\left[(1+\beta) \kappa^{-1}+1+\beta \kappa^{-1}\left(1+\eta_{y} \eta_{i}^{-1}\right)\right] E_{t} \hat{m}_{t+1} \\
& +\left[\kappa^{-1}+\eta_{i}^{-1}+1+(1+\beta) \kappa^{-1}\left(1+\eta_{y} \eta_{i}^{-1}\right)\right] \hat{m}_{t}-\kappa^{-1}\left(1+\eta_{y} \eta_{i}^{-1}\right) \hat{m}_{t-1}
\end{aligned}
$$




$$
\begin{aligned}
& -\frac{\xi_{b}}{\bar{m}} \beta \kappa^{-1} E_{t} \hat{b}_{t+1}+\frac{\xi_{b}}{\bar{m}}\left[\kappa^{-1}+1+\beta \kappa^{-1}\left(1+\eta_{y} \eta_{i}^{-1}\right)\right] \hat{b}_{t}-\frac{\xi_{b}}{\bar{m}} \kappa^{-1}\left(1+\eta_{y} \eta_{i}^{-1}\right) \hat{b}_{t-1} \\
= & \frac{1}{\bar{m}} \kappa^{-1}\left(1+\eta_{y} \eta_{i}^{-1}\right) \varepsilon_{s, t},
\end{aligned}
$$

which is further reduced to

$$
\begin{aligned}
& E_{t} \hat{m}_{t+2}-\left[\left(\beta^{-1}+1\right)+\beta^{-1} \kappa+\left(1+\eta_{y} \eta_{i}^{-1}\right)\right] E_{t} \hat{m}_{t+1} \\
& +\left[\beta^{-1}+\beta^{-1} \kappa\left(\eta_{i}^{-1}+1\right)+\left(\beta^{-1}+1\right)\left(1+\eta_{y} \eta_{i}^{-1}\right)\right] \hat{m}_{t}-\beta^{-1}\left(1+\eta_{y} \eta_{i}^{-1}\right) \hat{m}_{t-1} \\
= & \frac{\xi_{b}}{\bar{m}}\left\{\rho_{b}^{2}-\left[\beta^{-1}+\beta^{-1} \kappa+\left(1+\eta_{y} \eta_{i}^{-1}\right)\right] \rho_{b}+\beta^{-1}\left(1+\eta_{y} \eta_{i}^{-1}\right)\right\} \hat{b}_{t-1} \\
& +\left\{\frac{1}{\bar{m}} \beta^{-1}\left(1+\eta_{y} \eta_{i}^{-1}\right)+\frac{\xi_{b}}{\bar{m}} \beta^{-1}\left[-\rho_{b}+\beta^{-1}+\beta^{-1} \kappa+\left(1+\eta_{y} \eta_{i}^{-1}\right)\right]\right\} \varepsilon_{s, t},
\end{aligned}
$$

using (33).

Therefore, the set of the state variables of the model includes $\hat{m}_{t-1}$ and $\hat{b}_{t-1}$ only. Suppose that (36) has a unique stable solution for $\hat{m}_{t}{ }^{35}$ Given the state variables $\hat{m}_{t-1}$ and $\hat{b}_{t-1}$ and the shock $\varepsilon_{s, t}$ at the beginning of the period $t, \hat{m}_{t}, \hat{b}_{t}$, and $E_{t} \hat{m}_{t+1}$ are determined. We can in turn determine $\hat{Y}_{t}$ and $\hat{\pi}_{t}$ using (34) and (35).

\section{C.6 Proof}

\section{C.6.1 Proof of Proposition 7}

Under monetary dominance $\left(\xi_{b}=0\right)$, real money balance $\hat{m}_{t}$ is not dependent on government debt outstanding $\hat{b}_{t-1}$ as can be seen in (36). Also, $\hat{Y}_{t}$ and $\hat{\pi}_{t}$ evolve independently of $\hat{b}_{t}$ as seen in (34) and (35) if $\xi_{b}=0$. That is, under monetary dominance, the system becomes block-recursive and the private-sector equilibrium and monetary policy are determined independently of the fiscal block (given that a solution exists). This is not true in general and $\hat{b}_{t-1}$ is a state variable under fiscal dominance since $\xi_{b} \neq 0$.

\section{Quantitative Model}

In this section, we provide a complete description of the quantitative model and the details of the numerical exercise. We also present and discuss results with respect to non-policy shocks including neutral technology shock, government spending, investment specific technology shock, price markup shock, wage markup shock, and a preference shock. Results with respect to a change in the inflation target and public debt outstanding are presented in the main text.

\footnotetext{
${ }^{35}$ We numerically checked that (36) has a unique stable solution for $\hat{m}_{t}$ for any reasonable values of the structural parameters.
} 


\section{D.1 Model Description}

\section{D.1.1 Households}

There is a continuum of households in the unit interval. Each household specializes in the supply of a particular type of labor. A household that supplies labor of type- $j$ maximizes the utility function:

$$
E_{0}\left\{\sum_{t=0}^{\infty} \beta^{t} \delta_{t}\left[\log \left(C_{t}^{j}-\eta C_{t-1}\right)-\varpi \frac{\left(H_{t}^{j}\right)^{1+\varphi}}{1+\varphi}\right]\right\}
$$

where $H_{t}^{j}$ denotes the hours of type- $j$ labor services, $C_{t}$ is aggregate consumption, and $C_{t}^{j}$ is consumption of household $j$. The parameters $\beta, \varphi$, and $\eta$ are, respectively, the discount factor, the inverse of the (Frisch) elasticity of labor supply, and the degree of external habit formation, while $\delta_{t}$ represents an intertemporal preference shock that follows:

$$
\delta_{t}=\delta_{t-1}^{\rho_{\delta}} \exp \left(\varepsilon_{\delta, t}\right)
$$

where $\varepsilon_{\delta, t} \sim$ i.i.d. $N\left(0, \sigma_{\delta}^{2}\right)$.

Household $j$ 's flow budget constraint is:

$$
P_{t} C_{t}^{j}+P_{t} I_{t}^{j}+B_{t}^{j}+E_{t}\left[Q_{t, t+1} V_{t+1}^{j}\right]=W_{t}(j) H_{t}^{j}+V_{t}^{j}+R_{t-1} B_{t-1}^{j}+R_{t}^{k} u_{t} \bar{K}_{t-1}^{j}-P_{t} a\left(u_{t}\right) \bar{K}_{t-1}^{j}+\Pi_{t}-T_{t},
$$

where $P_{t}$ is the price level, $B_{t}^{j}$ is the amount of one-period risk-less nominal government bond held by household $j, R_{t}$ is the interest rate on the bond, $W_{t}(j)$ is the nominal wage rate for type- $j$ labor, $\Pi_{t}$ denotes profits of intermediate firms, and $T_{t}$ denotes government taxes. ${ }^{36}$ In addition to the government bond, households trade at time $t$ one-period state-contingent nominal securities $V_{t+1}^{j}$ at price $Q_{t, t+1}$, and hence fully insure against idiosyncratic risk.

Moreover, $I_{t}^{j}$ is investment, $R_{t}^{k}$ is the rental rate of effective capital $u_{t} \bar{K}_{t-1}^{j}$ where $u_{t}$ is the variable capacity utilization rate, and $a\left(u_{t}\right)$ is the cost of capital utilization. In steady-state, $u=1$ and $a(1)=0$. Moreover, in the first-order approximation of the model, the only parameter that matters for the dynamic solution of the model is the curvature $\chi \equiv \frac{a^{\prime \prime}(1)}{a^{\prime}(1)}$. The capital accumulation equation is then given by:

$$
\bar{K}_{t}^{j}=(1-d) \bar{K}_{t-1}^{j}+\mu_{t}\left(1-S\left(\frac{I_{t}^{j}}{I_{t-1}^{j}}\right)\right) I_{t}^{j},
$$

where $d$ is the depreciation rate and $S($.$) is the adjustment cost function. In steady-state, S=S^{\prime}=0$ and $S^{\prime \prime}>0 . \mu_{t}$ represents an investment shock that follows:

$$
\mu_{t}=\mu_{t-1}^{\rho_{\mu}} \exp \left(\varepsilon_{\mu, t}\right)
$$

where $\varepsilon_{\mu, t} \sim$ i.i.d. $N\left(0, \sigma_{\mu}^{2}\right)$.

Each household monopolistically provides differentiated labor. There are competitive employment agencies that assemble these differentiated labor into a homogenous labor input that is sold to intermediate goods firms. The assembling technology is a constant-elasticity-of-substitution (CES) production technology $H_{t}=$

\footnotetext{
${ }^{36}$ The budget constraint reflects our assumptions that each household owns an equal share of all intermediate firms and receives the same amount of net lump-sum transfers from the government.
} 
$\left(\int_{0}^{1} H_{t}^{j \frac{\theta_{l, t}-1}{\theta_{l, t}}} d j\right)^{\frac{\theta_{l, t}}{\theta_{l, t}-1}}$, where $\theta_{l, t}$ denotes the time-varying elasticity of substitution between differentiated labor. The corresponding wage index for the homogenous labor input is $W_{t}=\left(\int_{0}^{1} W_{t}(j)^{1-\theta_{l, t}} d j\right)^{\frac{1}{1-\theta_{l, t}}}$ and the optimal demand for $H_{t}^{j}$ is given by $H_{t}^{j}=\left(W_{t}(j) / W_{t}\right)^{-\theta_{l, t}} H_{t}$. The elasticity of substitution $\theta_{l, t}$ follows:

$$
\left(\frac{\theta_{l, t}}{\theta_{l, t}-1}\right)=\left(\frac{\bar{\theta}_{l}}{\bar{\theta}_{l}-1}\right)^{1-\rho_{l}}\left(\frac{\theta_{l, t-1}}{\theta_{l, t}-1}\right)^{\rho_{l}} \exp \left(\varepsilon_{l, t}-v_{l} \varepsilon_{l, t-1}\right)
$$

where $\varepsilon_{l, t} \sim$ i.i.d. $N\left(0, \sigma_{l}^{2}\right)$.

Each household resets its nominal wage optimally with probability $1-\alpha_{w}$ every period. Households that do not optimize adjust their wages according to the simple partial dynamic indexation rule:

$$
W_{t}(j)=W_{t-1}(j)\left[\pi_{t-1} a_{t-1}\right]^{\gamma_{w}}[\bar{\pi} \bar{a}]^{1-\gamma_{w}},
$$

where $\gamma_{w}$ measures the extent of indexation and $\bar{\pi}$ is the steady-state value of the gross inflation rate $\pi_{t} \equiv$ $P_{t} / P_{t-1}$. All optimizing households choose a common wage $W_{t}^{*}$ to maximize the present discounted value of future utility:

$$
E_{t} \sum_{k=0}^{\infty} \alpha_{w}^{k} \beta^{k}\left[-\delta_{t+k} \frac{\left(H_{t+k}^{j}\right)^{1+\varphi}}{1+\varphi}+\Lambda_{t+k} W_{t}^{*} H_{t+k}^{j}\right]
$$

where $\Lambda_{t+k}$ is the marginal utility of nominal income.

\section{D.1.2 Firms}

The final good $Y_{t}$, which is consumed by the government and households as well as used to invest, is produced by perfectly competitive firms assembling intermediate goods, $Y_{t}(i)$, with a CES production technology

$$
Y_{t}=\left(\int_{0}^{1} Y_{t}(i)^{\frac{\theta_{p, t}-1}{\theta_{p, t}}} d i\right)^{\frac{\theta_{p, t}}{\theta_{p, t}-1}}
$$

where $\theta_{p, t}$ denotes the elasticity of substitution between intermediate goods. The corresponding price index for the final consumption good is

$$
P_{t}=\left(\int_{0}^{1} P_{t}(i)^{1-\theta_{p, t}} d i\right)^{\frac{1}{1-\theta_{p, t}}}
$$

where $P_{t}(i)$ is the price of the intermediate good $i$. The optimal demand for $Y_{t}(i)$ is given by

$$
Y_{t}(i)=\left(\frac{P_{t}(i)}{P_{t}}\right)^{-\theta_{p, t}} Y_{t}
$$

The elasticity of substitution $\theta_{p, t}$ follows:

$$
\left(\frac{\theta_{p, t}}{\theta_{p, t}-1}\right)=\left(\frac{\bar{\theta}_{p}}{\bar{\theta}_{p}-1}\right)^{1-\rho_{p}}\left(\frac{\theta_{p, t-1}}{\theta_{p, t}-1}\right)^{\rho_{p}} \exp \left(\varepsilon_{p, t}-v_{p} \varepsilon_{p, t-1}\right)
$$

where $\varepsilon_{p, t} \sim$ i.i.d. $N\left(0, \sigma_{p}^{2}\right)$. 
Monopolistically competitive firms produce intermediate goods using the production function:

$$
Y_{t}(i)=\max \left\{\left(A_{t} H_{t}(i)\right)^{1-\lambda} K_{t}(i)^{\lambda}-A_{t} F ; 0\right\}
$$

where $H_{t}(i)$ and $K_{t}(i)$ denote the homogenous labor and capital employed by firm $i$ and $A_{t}$ represents exogenous economy-wide technological progress. The gross growth rate of technology $a_{t} \equiv A_{t} / A_{t-1}$ follows:

$$
a_{t}=\bar{a}^{1-\rho_{a}} a_{t-1}^{\rho_{a}} \exp \left(\varepsilon_{a, t}\right)
$$

where $\bar{a}$ is the steady-state value of $a_{t}$ and $\varepsilon_{a, t} \sim$ i.i.d. $N\left(0, \sigma_{a}^{2}\right) . F$ is a fixed cost of production that ensure that profits are zero in steady state.

A firm resets its price optimally with probability $1-\alpha_{p}$ every period. Firms that do not optimize adjust their price according to the simple partial dynamic indexation rule:

$$
P_{t}(i)=P_{t-1}(i) \pi_{t-1}^{\gamma_{p}} \bar{\pi}^{1-\gamma_{p}}
$$

where $\gamma_{p}$ measures the extent of indexation and $\bar{\pi}$ is the steady-state value of the gross inflation rate $\pi_{t} \equiv$ $P_{t} / P_{t-1}$. All optimizing firms choose a common price $P_{t}^{*}$ to maximize the present discounted value of future profits:

$$
E_{t} \sum_{k=0}^{\infty} \alpha_{p}^{k} Q_{t, t+k}\left[P_{t}^{*} X_{t, k} Y_{t+k}(i)-W_{t+k} H_{t+k}(i)-R_{t+k}^{k} K_{t+k}(i)\right]
$$

where

$$
X_{t, k} \equiv\left\{\begin{array}{c}
\left(\pi_{t} \pi_{t+1} \cdots \pi_{t+k-1}\right)^{\gamma} \bar{\pi}^{(1-\gamma) k}, \quad k \geq 1 \\
1, \quad k=0
\end{array}\right.
$$

\section{D.1.3 Government}

Budget Constraint Each period, the government collects lump-sum tax revenues $T_{t}$ and issues oneperiod nominal bonds $B_{t}$ to finance its consumption $G_{t}$, and interest payments. Accordingly, the flow budget constraint is given by:

$$
\frac{B_{t}}{P_{t}}=R_{t-1} \frac{B_{t-1}}{P_{t}}+G_{t}-T_{t}
$$

The flow budget constraint can be rewritten as:

$$
R_{t}^{-1} b_{t}=b_{t-1} \frac{1}{\pi_{t}} \frac{y_{t-1}}{y_{y}} \frac{A_{t-1}}{A_{t}}+\tilde{G}_{t}-\tau_{t}
$$

where $b_{t} \equiv R_{t} \frac{B_{t}}{P_{t} Y_{t}}$ denotes the real maturity value of government debt relative to output, $\tilde{G}_{t} \equiv \frac{G_{t}}{Y_{t}}$, and $\tau_{t}$ $\equiv \frac{T_{t}}{Y_{t}}$.

Monetary Policy The central bank sets the nominal interest rate according to a Taylor-type rule:

$$
\frac{R_{t}}{\bar{R}}=\left(\frac{R_{t-1}}{\bar{R}}\right)^{\rho_{R}}\left[\left(\frac{\pi_{t}}{\pi_{t}^{*}}\right)^{\phi_{\pi}}\left(\frac{X_{t}}{X_{t}^{*}}\right)^{\phi_{Y}}\right]^{1-\rho_{R}} \exp \left(\varepsilon_{R, t}\right),
$$


which features interest rate smoothing and systematic responses to deviation of GDP from its natural level $X_{t}^{*}$ and deviation of inflation from a time-varying target $\pi_{t}^{*} \cdot{ }^{37} \quad \bar{R}$ is the steady-state value of $R_{t}$ and the non-systematic monetary policy shock $\varepsilon_{R, t}$ is assumed to follow i.i.d. $N\left(0, \sigma_{R}^{2}\right)$. The inflation target evolves exogenously as:

$$
\pi_{t}^{*}=\bar{\pi}^{1-\rho_{\pi}}\left(\pi_{t-1}^{*}\right)^{\rho_{\pi}} \exp \left(\varepsilon_{\pi, t}\right)
$$

where $\varepsilon_{\pi, t} \sim$ i.i.d. $N\left(0, \sigma_{\pi}^{2}\right)$.

Fiscal Policy The fiscal authority sets the tax revenues according to:

$$
\frac{\tau_{t}}{\bar{\tau}}=\left(\frac{\tau_{t-1}}{\bar{\tau}}\right)^{\rho_{\tau}}\left[\left(\frac{b_{t-1}}{\bar{b}}\right)^{\tilde{\psi}}\right]^{1-\rho_{\tau}},
$$

which features tax smoothing and a systematic response to lagged debt. $\bar{\tau}$ is the steady-state value of $\tau_{t}$ while $\bar{b}$ is the steady-state value of $b_{t}$. Government spending follows an exogenous process given by:

$$
G_{t}=\left(1-\frac{1}{g_{t}}\right) Y_{t}
$$

where the government spending shock follows:

$$
g_{t}=\bar{g}^{1-\rho_{g}} g_{t-1}^{\rho_{g}} \exp \left(\varepsilon_{g, t}\right),
$$

where $\varepsilon_{g, t} \sim$ i.i.d. $N\left(0, \sigma_{g}^{2}\right)$.

\section{D.1.4 Equilibrium}

Equilibrium is characterized by the prices and quantities that satisfy the households' and firms' optimality conditions, the government budget constraint, monetary and fiscal policy rules, and the clearing conditions for the product, labor, and asset markets:

$$
\begin{array}{r}
\int_{0}^{1} C_{t}^{j} d j+G_{t}+\int_{0}^{1} I_{t}^{j} d j+a\left(u_{t}\right) \int_{0}^{1} \bar{K}_{t-1}^{j} d j=Y_{t} \\
\int_{0}^{1} H_{t}(i) d i=H_{t} \\
\int_{0}^{1} V_{t}^{j} d j=0 \\
\int_{0}^{1} B_{t}^{j} d j=B_{t} .
\end{array}
$$

Note that $C_{t}^{j}=C_{t}, I_{t}^{j}=I_{t}$, and $\bar{K}_{t-1}^{j}=\bar{K}_{t-1}$ due to the complete market assumption and the separability between consumption and leisure. The capital accumulation equation in the aggregate is then given by:

$$
\bar{K}_{t}=(1-d) \bar{K}_{t-1}+\mu_{t}\left[1-S\left(\frac{I_{t}}{I_{t-1}}\right)\right] I_{t} .
$$

and the aggregate resource constraint and the definition of GDP then take the form:

\footnotetext{
${ }^{37}$ The natural level of output is the output that would prevail under flexible wages and prices and in the absence of time-variation in the elasticity of subsitution over differentiated labor and goods varieties.
} 


$$
C_{t}+I_{t}+G_{t}+a\left(u_{t}\right) \bar{K}_{t-1}=Y_{t}
$$

$$
X_{t}=C_{t}+I_{t}+G_{t} .
$$

\section{D.2 Approximate Model}

We first solve the problem of households and firms given the monetary and fiscal policy rules and derive the equilibrium conditions. We then use approximation methods to solve the model. First, the model features a stochastic balanced growth path since the neutral technology shock contains a unit root. Therefore, we de-trend variables on the balanced growth path by the level of the technology shock and write down all the equilibrium conditions of the transformed model. Second, we compute the non-stochastic steady state of this transformed model. Third, we obtain a first-order approximation of the equilibrium conditions around this steady state. We then solve the approximated model using standard methods.

For a variable $X_{t}$, let $x_{t}=\frac{X_{t}}{A_{t}}$. We denote by $\hat{x}_{t}$ the log-deviation from steady state of $x_{t}$, except for fiscal variables, which are in terms of deviation from steady state.

We also, define some new variables: $\lambda_{t}=\Lambda_{t} P_{t} A_{t}$, and $\phi_{t}$, which is the lagrange multiplier on the capital accumulation equation for the household's optimization problem (that is, it is the shadow value of installed capital), $\varrho_{p, t}=\frac{\theta_{p, t}}{\theta_{p, t}-1}$, and $\varrho_{l, t}=\frac{\theta_{l, t}}{\theta_{l, t}-1}$. We omit a detailed derivation and the equations characterizing the approximate equilibrium, after some manipulations, are given by:

$$
\begin{aligned}
\hat{y}_{t}= & \frac{\bar{y}+F}{\bar{y}}\left[\lambda \hat{k}_{t}+(1-\lambda) \hat{H}_{t}\right] \\
\chi \hat{u}_{t}= & \hat{w}_{t}+\hat{L}_{t}-\hat{k}_{t} \\
\hat{\pi}_{t}= & \frac{\beta}{1+\beta \gamma_{p}} E_{t} \hat{\pi}_{t+1}+\frac{\gamma_{p}}{1+\beta \gamma_{p}} \hat{\pi}_{t-1}+\kappa_{p}\left[\lambda\left(\chi \hat{u}_{t}\right)+(1-\lambda) \hat{w}_{t}\right]+\kappa_{p} \hat{\varrho}_{p, t} \\
\hat{\lambda}_{t}= & \hat{R}_{t}+E_{t}\left(\hat{\lambda}_{t+1}-\hat{a}_{t+1}-\hat{\pi}_{t+1}\right) \\
\hat{\lambda}_{t}= & \frac{\eta \beta \bar{a}}{(\bar{a}-\eta \beta)(\bar{a}-\eta)} E_{t} \hat{c}_{t+1}-\frac{\bar{a}^{2}+\eta^{2} \beta}{(\bar{a}-\eta \beta)(\bar{a}-\eta)} \hat{c}_{t}+\frac{\eta \bar{a}}{(\bar{a}-\eta \beta)(\bar{a}-\eta)} \hat{c}_{t-1} \\
& +\frac{\eta \beta \bar{a} \rho_{a}-\eta \bar{a}}{(\bar{a}-\eta \beta)(\bar{a}-\eta)} \hat{a}_{t}+\frac{\left(\bar{a}-\eta \beta \rho_{\delta}\right)}{(\bar{a}-\eta \beta)} \hat{\delta}_{t} \\
\hat{\phi}_{t}= & (1-d) \beta \bar{a}^{-1} E_{t}\left(\hat{\phi}_{t+1}-\hat{a}_{t+1}\right)+\left(1-(1-d) \beta \bar{a}^{-1}\right) E_{t}\left[\hat{\lambda}_{t+1}-\hat{a}_{t+1}+\chi \hat{u}_{t+1}\right] \\
\hat{\lambda}_{t}= & \hat{\phi}_{t}+\hat{\mu}_{t}-\bar{a}^{2} S^{\prime \prime}\left(\hat{\imath}_{t}-\hat{\imath}_{t-1}+\hat{a}_{t}\right)+\beta \bar{a}^{2} S^{\prime \prime} E_{t}\left[\hat{\imath}_{t+1}-\hat{\imath}_{t}+\hat{a}_{t+1}\right] \\
\hat{k}_{t}= & \hat{u}_{t}+\hat{\bar{k}}_{t-1}-\hat{a}_{t} \\
\widehat{\bar{k}}_{t}= & (1-d) \bar{a}^{-1}\left(\overline{\bar{k}}_{t-1}-\hat{a}_{t}\right)+\left(1-(1-d) \bar{a}^{-1}\right)\left(\hat{\mu}_{t}+\hat{u}_{t}\right) \\
\hat{w}_{t}= & \frac{1}{1+\beta} \hat{w}_{t-1}+\frac{\beta}{1+\beta} E_{t} \hat{w}_{t+1}-\kappa_{w}\left[\hat{w}_{t}-\left(\varphi L_{t}+\hat{\delta}_{t}-\hat{\lambda}_{t}\right)\right] \\
& +\frac{\gamma_{w}}{1+\beta} \hat{\pi}_{t-1}-\frac{1+\beta \gamma_{p}}{1+\beta} \hat{\pi}_{t}+\frac{\beta}{1+\beta} E_{t} \hat{\pi}_{t+1}+\frac{\gamma_{w}}{1+\beta} \hat{a}_{t-1}-\frac{1+\beta \gamma_{w}-\beta \rho_{a}}{1+\beta} \hat{a}_{t}+\kappa_{w} \hat{\varrho}_{l, t} \\
\hat{R}_{t}= & \rho_{R} \hat{R}_{t-1}+\left(1-\rho_{R}\right)\left[\phi_{\pi}\left(\pi_{t}-\pi_{t}^{*}\right)+\phi_{y}\left(x_{t}-x_{t}^{*}\right)\right]+\varepsilon_{R, t} \\
\hat{x}_{t}= & \hat{y}_{t}-\frac{\rho \bar{k}}{\bar{y}} \hat{u}_{t}
\end{aligned}
$$




$$
\begin{aligned}
\frac{1}{\bar{g}} \hat{y}_{t} & =\frac{1}{\bar{g}} \hat{g}_{t}+\frac{\bar{c}}{\bar{y}} \hat{c}_{t}+\frac{\bar{c}}{\bar{y}} \hat{\imath}_{t}+\frac{\rho \bar{k}}{\bar{y}} \hat{u}_{t} \\
\hat{b}_{t} & =\beta^{-1} \hat{b}_{t-1}-\beta^{-1} \bar{b}\left[\hat{\pi}_{t}+\hat{y}_{t}-\hat{y}_{t-1}+\hat{a}_{t}\right]-\bar{R} \hat{\tau}_{t}+\bar{b} \hat{R}_{t}+\bar{R} \frac{1}{\bar{g}} \hat{g}_{t} \\
\hat{\tau}_{t} & =\rho_{\tau} \hat{\tau}_{t-1}+\left(1-\rho_{\tau}\right) \psi \hat{b}_{t-1}
\end{aligned}
$$

where $\rho=\frac{\bar{a}}{\beta}-(1-d), \kappa_{p}=\frac{\left(1-\beta \alpha_{p}\right)\left(1-\alpha_{p}\right)}{\alpha_{p}\left(1+\gamma_{p} \beta\right)}, \quad \kappa_{w}=\frac{\left(1-\beta \alpha_{w}\right)\left(1-\alpha_{w}\right)}{\alpha_{w}(1+\beta)\left(1+\varphi \theta_{l}\right)}$, and $\psi \equiv \overline{\bar{\tau}} \tilde{\bar{\psi}}$. 


\section{D.3 Parameterization}

Table 1: Benchmark Parameterization

\begin{tabular}{|c|c|c|}
\hline Parameter & Value & Description \\
\hline$\lambda$ & 0.17 & Capital share \\
\hline$d$ & 0.025 & Depreciation rate of capital \\
\hline$\gamma_{p}$ & 0.24 & Price indexation \\
\hline$\gamma_{w}$ & 0.11 & Wage indexation \\
\hline $100 \log \bar{a}$ & 0.48 & SS technology growth rate \\
\hline$\eta$ & 0.78 & Consumption habit \\
\hline $1 /\left(1-\bar{\theta}_{p}\right)$ & 0.23 & SS price markup \\
\hline $1 /\left(1-\bar{\theta}_{l}\right)$ & 0.15 & SS wage markup \\
\hline $\log \bar{H}$ & 0.38 & SS log-hours \\
\hline $100(\bar{\pi}-1)$ & 0.71 & SS quarterly inflation \\
\hline $100\left(\beta^{-1}-1\right)$ & 0.13 & Discount factor \\
\hline$\varphi$ & 3.79 & Inverse Frisch elasticity \\
\hline$\alpha_{p}$ & 0.84 & Calvo prices \\
\hline$\alpha_{w}$ & 0.70 & Calvo wages \\
\hline$\chi$ & 5.30 & Elasticity capital utilization costs \\
\hline$S^{\prime \prime}$ & 2.85 & Investment adjustment costs \\
\hline$\rho_{R}$ & 0.82 & Monetary rule smoothing \\
\hline$\rho_{\tau}$ & 0.80 & Fiscal rule smoothing \\
\hline$\rho_{a}$ & 0.23 & Neutral technology growth \\
\hline$\rho_{g}$ & 0.99 & Government spending \\
\hline$\rho_{\mu}$ & 0.72 & Investment \\
\hline$\rho_{p}$ & 0.94 & Price markup \\
\hline$\rho_{l}$ & 0.97 & Wage markup \\
\hline$\rho_{\delta}$ & 0.67 & Intertemporal preference \\
\hline$v_{p}$ & 0.77 & Price markup MA \\
\hline$v_{l}$ & 0.91 & Wage markup MA \\
\hline $100 \sigma_{R}$ & 0.22 & Monetary policy \\
\hline $100 \sigma_{a}$ & 0.88 & Neutral technology growth \\
\hline $100 \sigma_{g}$ & 0.35 & Government spending \\
\hline $100 \sigma_{\mu}$ & 6.03 & Investment \\
\hline $100 \sigma_{p}$ & 0.14 & Price markup \\
\hline $100 \sigma_{w}$ & 0.20 & Wage markup \\
\hline $100 \sigma_{\delta}$ & 0.04 & Intertemporal preference \\
\hline $\bar{b}$ & 0.40 & SS public debt \\
\hline $\bar{g}$ & 0.22 & SS government spending \\
\hline$\phi_{y}$ & 0.07 & Monetary rule output \\
\hline$\phi_{\pi}$ & {$[0,3]$} & Monetary rule inflation \\
\hline$\psi$ & {$[-0.1,0.1]$} & Fiscal rule public debt \\
\hline
\end{tabular}

Note: The benchmark values of the parameters other than $\rho_{\tau}, \bar{b}, \phi_{\pi}$ and $\psi$ are the estimated median of Justiniano, Primiceri and Tambalotti (2010). The value of $\rho_{\tau}$ is taken from Bhattarai, Lee and Park (2012) and $\bar{b}$ is the sample average ratio of public debt to output in the U.S. For policy parameters $\phi_{\pi}$ and $\psi$, we try different values for comparative statics. 


\section{D.4 Results}

\section{D.4.1 A Change in the Inflation Target and Debt Outstanding}

Results with respect to a change in the inflation target are presented in the main text.

\section{D.4.2 Non-policy Shocks}

Figure 11 shows under AMPF the impulse response of inflation to six non-policy shocks, varying the degree of monetary policy stance. It is clear that inflation responds less on impact to a non-policy shock when the systematic response of monetary policy to inflation is higher for all cases, except for the investment specific technology shock. Even for this shock however, after 5 periods or so, the response is smaller in magnitude for a greater $\phi_{\pi}$. In our simple model, where we abstract from investment, this shock is not present. In this quantitative model, the initial response of inflation is higher for a greater $\phi_{\pi}$ because this shock directly and significantly affects the capital rental cost for firms. Thus, when $\phi_{\pi}$ is greater, it can be the case that the rise in marginal cost due to a positive investment specific shock outweighs the usual inflation stabilization effect, thereby leading to a greater response of inflation. This result however, depends on all the other parameters of the model, in particular, the extent of wage stickiness in the economy. This is because wage stickiness determines the dynamics of wages, an important component of marginal cost. In fact in Figure 12 , in an alternate parameterization, we show a case where inflation responds less on impact to this shock with a greater $\phi_{\pi}$, which makes it consistent with our analytical results. In this alternate parameterization, we decrease the extent of wage stickiness compared to the baseline case presented here, which magnifies the inflation stabilization effect of monetary policy on wage costs and, thereby, damps down the increase in inflation following an investment specific shock.

Figure 13 shows under PMAF the impulse response of inflation to six non-policy shocks, varying the degree of monetary policy stance. It is clear that for all cases and in sharp contrast to AMPF, inflation responds more on impact to a non-policy shock when the systematic response of monetary policy to inflation is higher.

Figure 14 shows under PMAF the impulse response of inflation to six non-policy shocks, varying the degree of fiscal policy stance. It is clear that inflation responds less on impact to a non-policy shock when the systematic response of fiscal policy to debt is lower for all cases, except for the neutral technology shock. This result is different from our analytical results in the simple model. The reason is that this quantitative model features stochastic growth and the technology shock is therefore a shock to the growth rate as opposed to a shock to the level of technology, which was the case in the simple model. Thus, due to this, the shock can significantly affect the dynamics of inflation as it plays a prominent role in the government budget constraint. We do not present impulses responses under PMPF as the results clearly depend on the calibration of $M_{\pi}$. 

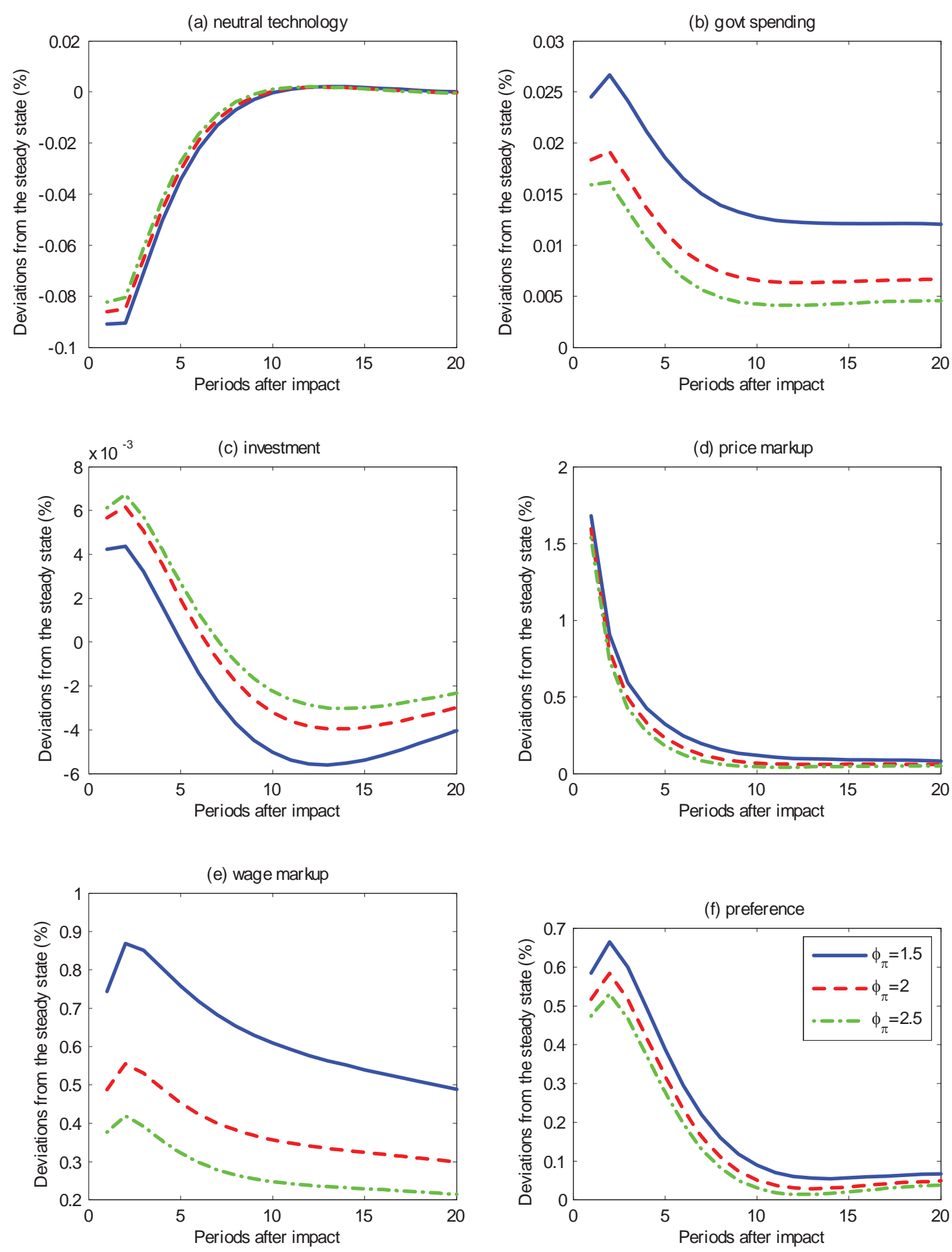

Figure 11: The response of inflation to a one percent increase in the non-policy shock under AMPF. 


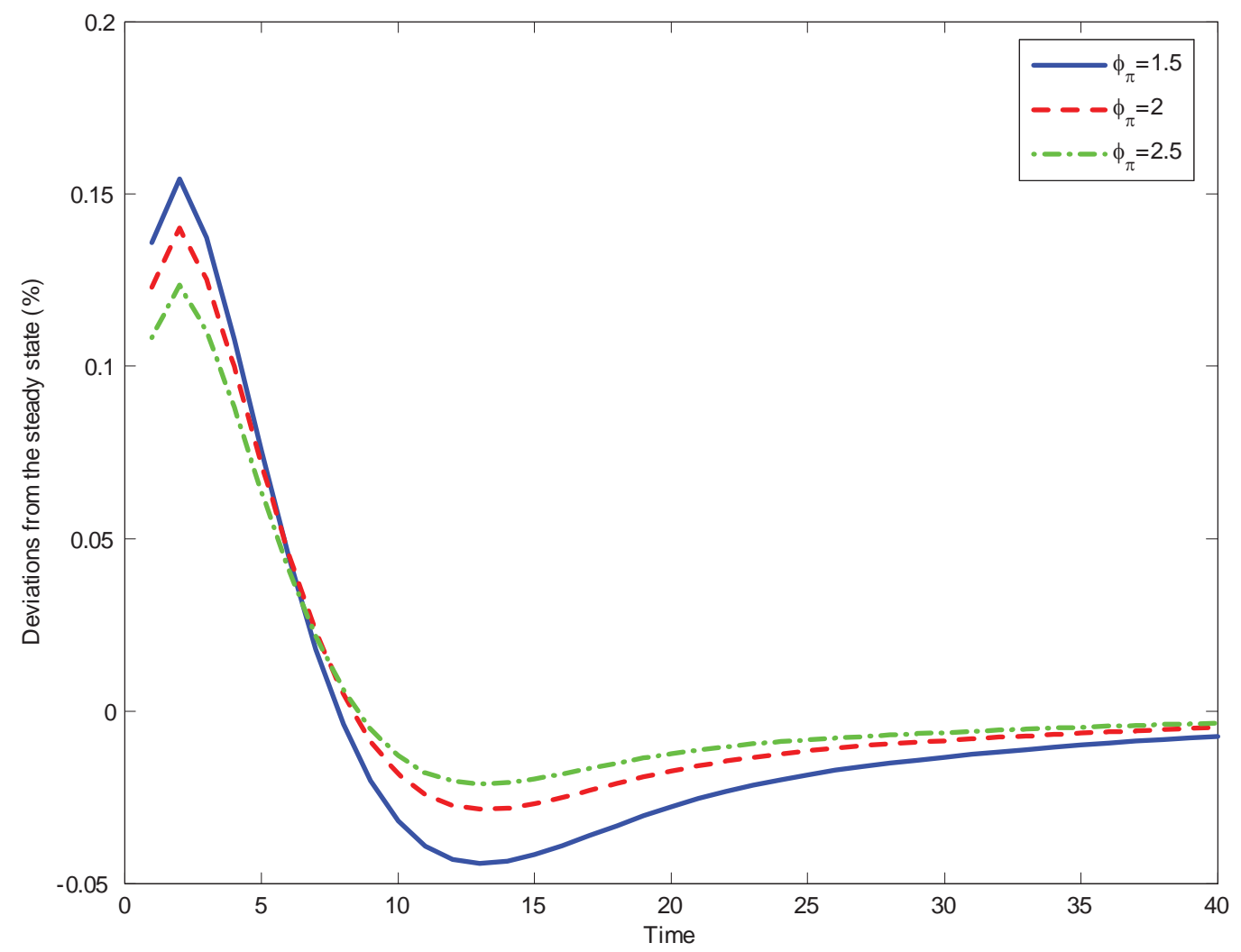

Figure 12: The response of inflation to a one percent increase in the investment specific shock under an alternate parameterization $\left(\alpha_{w}=0.3\right)$. 
(a) neutral technology

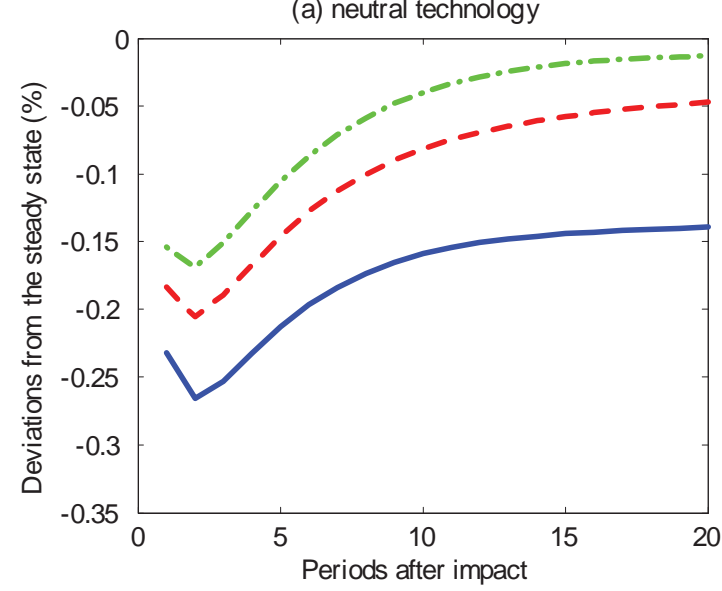

(c) investment

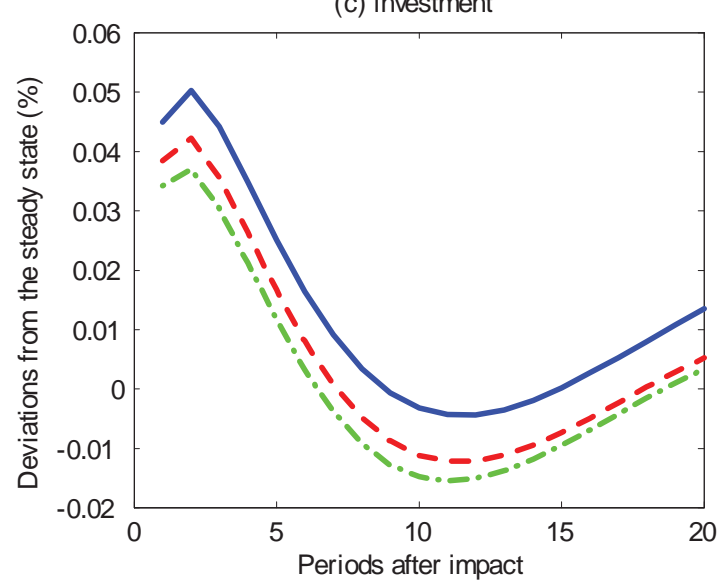

(e) wage markup

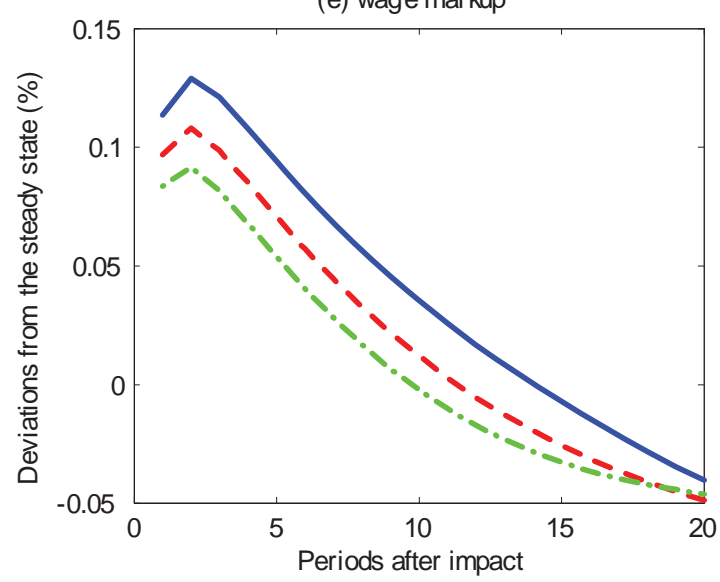

(b) govt spending

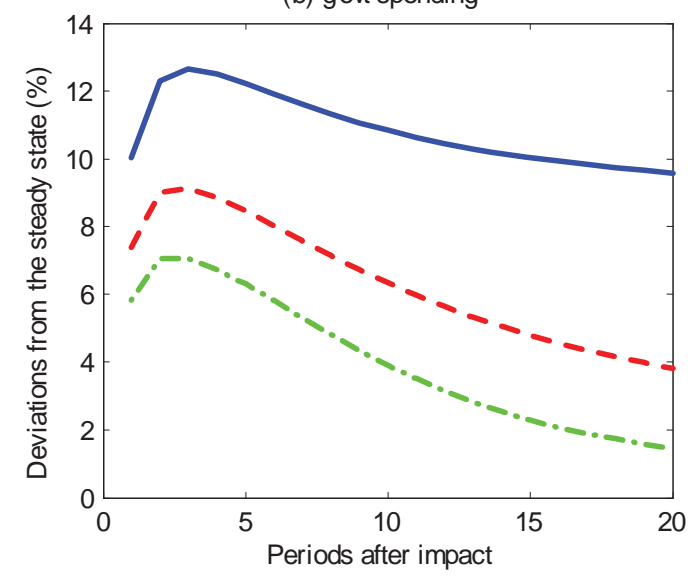

(d) price markup
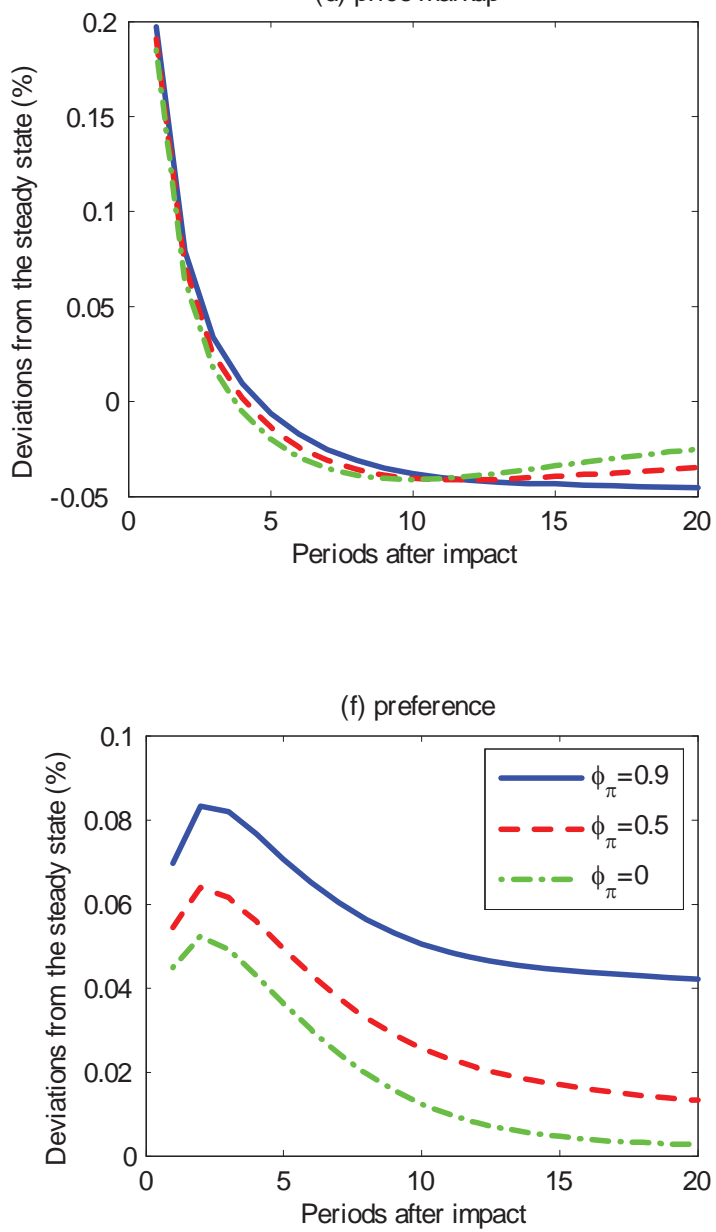

Figure 13: The response of inflation to a one percent increase in the non-policy shock under PMAF. 
(a) neutral technology

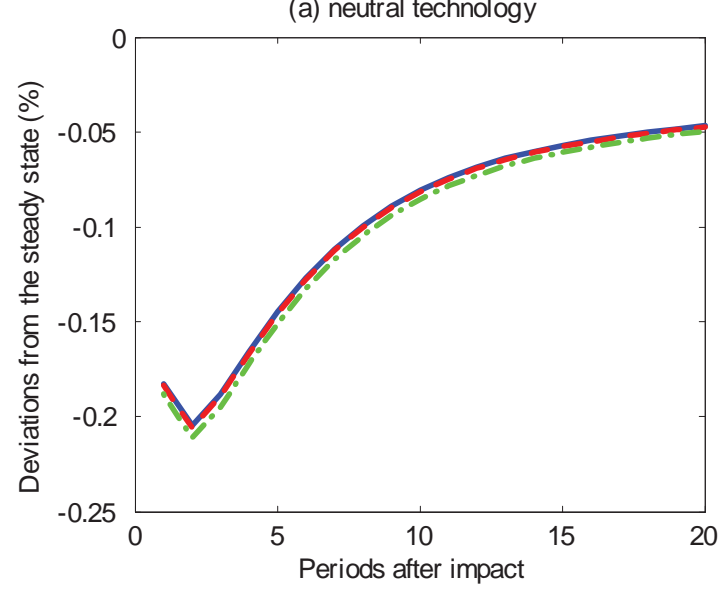

(c) investment

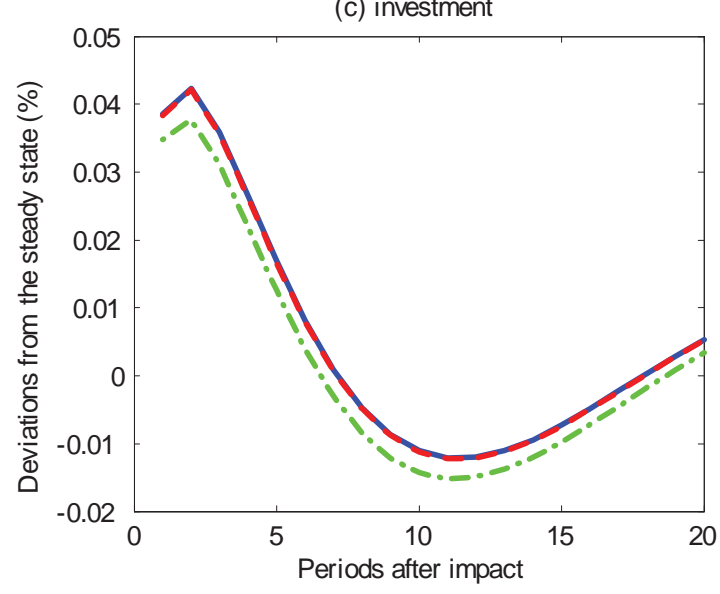

(e) wage markup

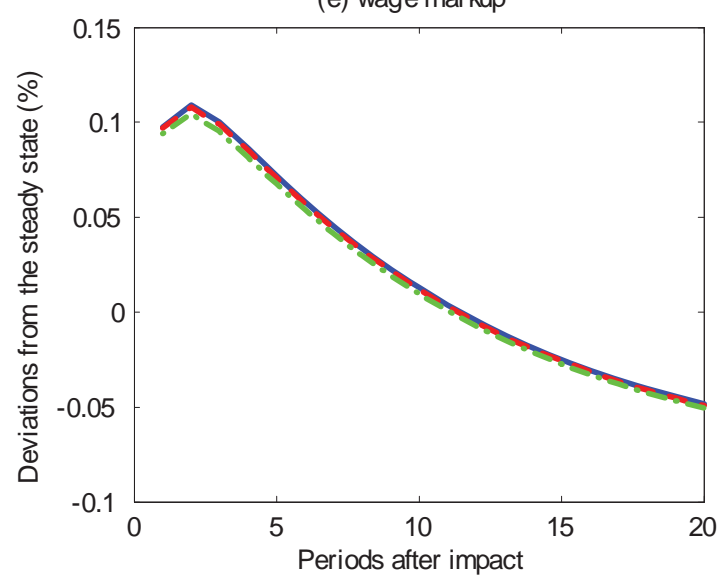

(b) govt spending

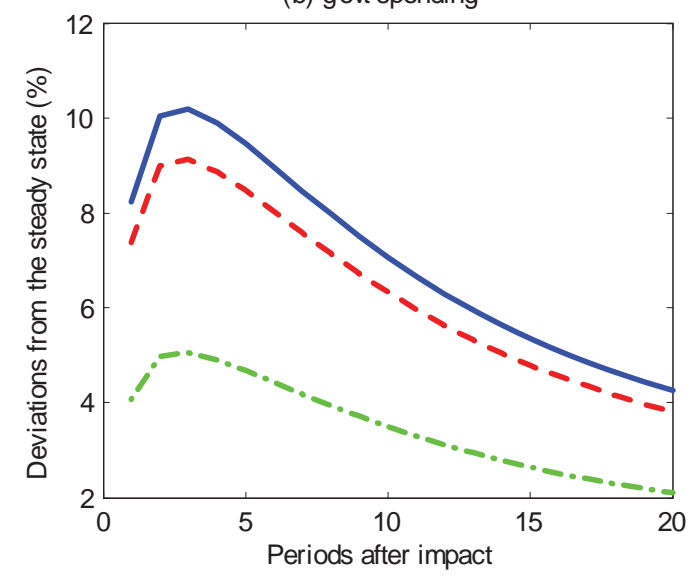

(d) price markup
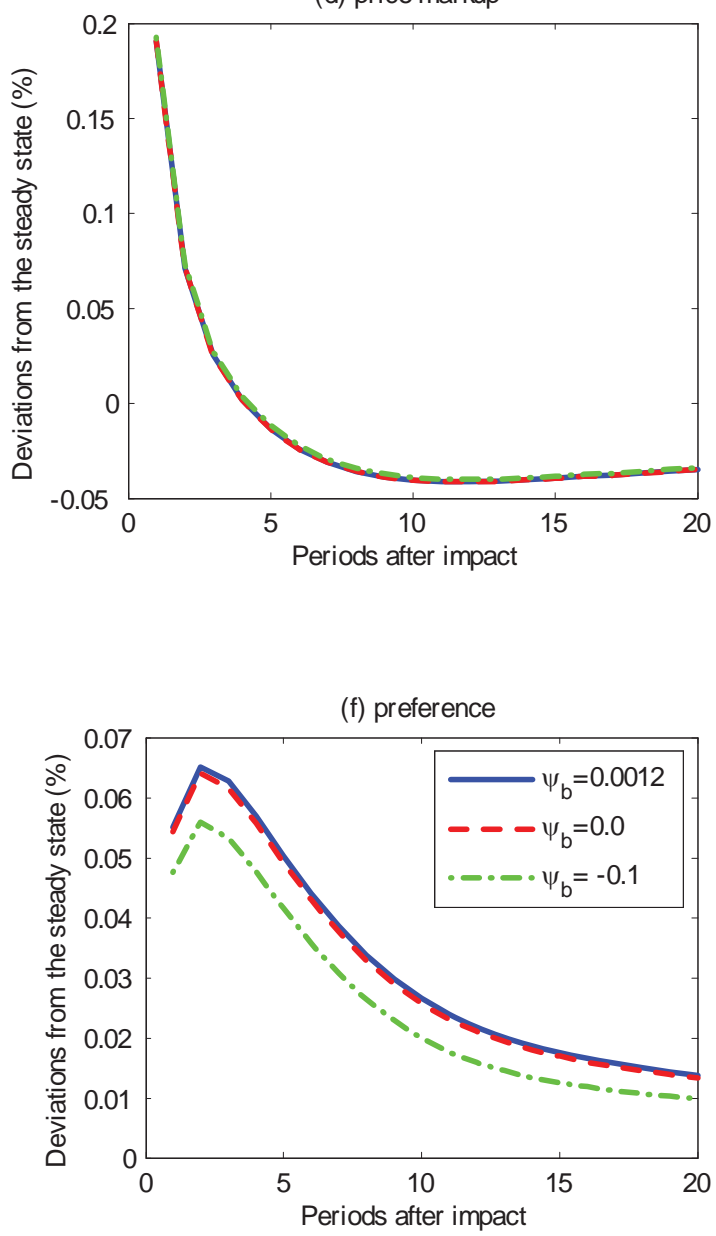

Figure 14: The response of inflation to a one percent increase in the non-policy shock under PMAF. 